\title{
TWO-DIMENSIONAL QUANTUM ANTIFERROMAGNETS
}

\author{
Grégoire Misguich \\ Institut de Physique Théorique, \\ CEA, IPhT, F-91191 Gif-sur-Yvette, France \\ CNRS, URA 2306, F-91191 Gif-sur-Yvette, France \\ E-mail: gregoire.misguich@cea.fr \\ Claire Lhuillier \\ Laboratoire de Physique Théorique de la matière condensée \\ Université P. et M. Curie and UMR 7600 of CNRS \\ Case 121, 4 Place Jussieu, 75252 Paris Cedex, France \\ E-mail: claire.lhuillier@upmc.fr
}

\begin{abstract}
This review presents some theoretical advances in the field of quantum magnetism in two-dimensional systems, and quantum spin liquids in particular. It is to be published as a chapter in the second edition of the book "Frustrated spin systems", edited by H. T. Diep (World-Scientific). The section (Sec. 7) devoted to the kagome antiferromagnet has been completely rewritten/updated, as well as the concluding section (Sec. 8). The other sections (Secs. 1-6) are unchanged from the first edition of the book (published in 2005).
\end{abstract}

\section{Contents}

1 Introduction . . . . . . . . . . . . . . . . . . . 3

$2 J_{1}-J_{2}$ model on the square lattice $\ldots \ldots \ldots \ldots$

2.1 Classical ground state and spin-wave analysis . . . . . . . . . 5

2.2 Order by disorder $\left(J_{2}>J_{1} / 2\right) \ldots \ldots \ldots \ldots$

2.3 Non-magnetic region $\left(J_{2} \simeq J_{1} / 2\right) \ldots \ldots \ldots \ldots$

2.3.1 Series expansions . . . . . . . . . . . . . . 8

2.3.2 Exact diagonalizations . . . . . . . . . . . . 10

2.3.3 Quantum Monte Carlo . . . . . . . . . . . . 11

3 Valence-bond crystals . . . . . . . . . . . . . . . . 12 
3.1 Definitions . . . . . . . . . . . . . . . . . . 12

3.2 One-dimensional and quasi one-dimensional examples (spin- $\frac{1}{2}$ sys-

tems $\ldots \ldots \ldots \ldots \ldots$. . . . . . . . . . . . . . . . . . . . . . . .

3.3 Valence Bond Solids . . . . . . . . . . . . . . . . . . . . 14

3.4 Two-dimensional examples of VBC . . . . . . . . . . . . 15

3.4.1 Without spontaneous lattice symmetry breaking . . . . . 15

3.4.2 With spontaneous lattice symmetry breaking . . . . . . . 17

3.5 Methods . . . . . . . . . . . . . . . . . . . . 19

3.6 Summary of the properties of VBC phases . . . . . . . . . . . 20

4 Large- $N$ methods . . . . . . . . . . . . . . . . . . . 22

4.1 Bond variables . . . . . . . . . . . . . . . . . . . . . . 22

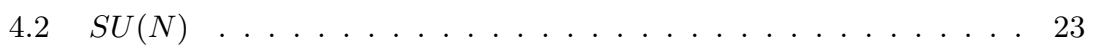

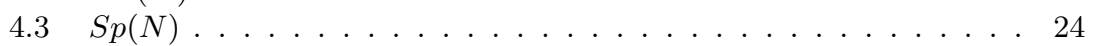

4.3.1 Gauge invariance ................ . . 25

4.3.2 Mean-field $(N=\infty$ limit $) \quad \ldots . . . . . . . . .26$

4.3.3 Fluctuations about the mean-field solution . . . . . . . . 26

4.3.4 Topological effects - instantons and spontaneous dimerization 28

4.3.5 Deconfined phases . . . . . . . . . . . . . . . 29

5 Quantum Dimer Models . . . . . . . . . . . . . . . . 30

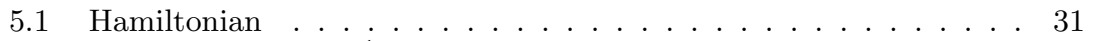

5.2 Relation with spin- $\frac{1}{2}$ models . . . . . . . . . . . . . 31

5.3 Square lattice . . . . . . . . . . . . . . . . . . . 33

5.3.1 Transition graphs and topological sectors . . . . . . . . 33

5.3 .2 Staggered VBC for $V / J>1 \ldots \ldots \ldots$

5.3.3 Columnar crystal for $V<0 \ldots \ldots \ldots$. . . . . . . 35

5.3.4 Plaquette phase .................. 35

5.3.5 Rokhsar-Kivelson point . . . . . . . . . . . . 36

5.4 Hexagonal lattice . . . . . . . . . . . . . . . . 37

5.5 Triangular lattice . . . . . . . . . . . . . . . . . 38

5.5.1 RVB liquid at the RK point . . . . . . . . . . . . 39

5.5 .2 Topological order . . . . . . . . . . . . . . . . 39

5.6 Solvable QDM on the kagome lattice . . . . . . . . . . . . . 40

5.6 .1 Hamiltonian . . . . . . . . . . . . . . . . . . 40

5.6 .2 RK ground state . . . . . . . . . . . . . . 41

5.6 .3 Ising pseudo-spin variables . . . . . . . . . . . . . . . 42

5.6 .4 Dimer-dimer correlations . . . . . . . . . . . . . 43

5.6 .5 Visons excitations ................ . . . . 44

5.6 .6 Spinons deconfinement . . . . . . . . . . . . . . . . . . . . .

$5.6 .7 \quad \mathbb{Z}_{2}$ gauge theory .................. 47

5.7 A QDM with an extensive ground state entropy . . . . . . . . 47

6 Multiple-spin exchange models . . . . . . . . . . . . . . . 48

6.1 Physical realizations of multiple-spin interactions . . . . . . . . 48

6.1.1 Nuclear magnetism of solid ${ }^{3} \mathrm{He}$. . . . . . . . . . . . . 48

6.1 .2 Wigner crystal . . . . . . . . . . . . . . 50 50

6.1.3 Cuprates .................... 51

6.2 Two-leg ladders . . . . . . . . . . . . . . . . 51 
6.3 MSE model on the square lattice . . . . . . . . . . . . . 553

6.4 RVB phase of the triangular $J_{2}-J_{4} \mathrm{MSE} \ldots \ldots \ldots \ldots$

6.4.1 Non-planar classical ground states . . . . . . . . . 554

6.4.2 Absence of Néel LRO . . . . . . . . . . . . . . . . . 554

6.4.3 Local singlet-singlet correlations - absence of lattice sym-

metry breaking. . . . . . . . . . . . 55

6.4.4 Topological degeneracy and Lieb-Schultz-Mattis Theorem . 55

6.4.5 Deconfined spinons . . . . . . . . . . . . . . 57

6.5 Other models with MSE interactions . . . . . . . . . . . . . 58

7 Antiferromagnets on the kagome lattice . . . . . . . . . . . . . . 58

7.1 Ising model . . . . . . . . . . . . . . . . 559

7.2 Classical Heisenberg models on the kagome lattice . . . . . . . . 59

7.3 Nearest-neighbor RVB description of the spin- $\frac{1}{2}$ kagome antiferromagnet . . . . . . . . . . . . . . . 60 60

7.4 Spin- $\frac{1}{2}$ Heisenberg model on the kagome lattice: numerics . . . . 62

7.4 .1 Ground-state energy per spin . . . . . . . . . . 62 62

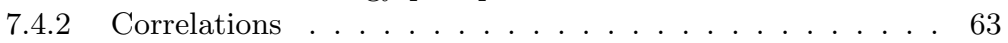

7.4 .3 Spin gap .................. . . . . . . . . . . . . . . . . .

7.4 .4 Singlet gap . . . . . . . . . . . . . . 63 63

7.4.5 Entanglement entropy and signature of a $\mathbb{Z}_{2}$ liquid . . . . . 64

7.4.6 Spin liquids on the kagome lattice and Projective symme-

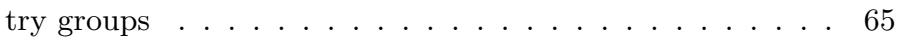

7.5 Competing phases ................... 67 67

7.5.1 Valence Bond Crystals . . . . . . . . . . . . . . 67 67

7.5.2 $U(1)$ Dirac Spin Liquid . . . . . . . . . . . . . . . 68 68

7.5.3 Spontaneously breaking the time-reversal symmetry, "chi-

ral" spin liquids . . . . . . . . . . . . . . . . 68

7.6 Experiments in compounds with kagome-like lattices . . . . . . . . 69

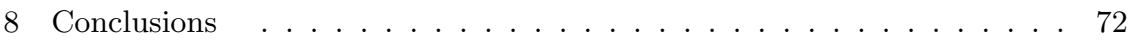

References . . . . . . . . . . . . . . . . . . 75

\section{Introduction}

In this review we present some theoretical advances in the field of quantum magnetism in two-dimensional (2D) systems. The spin- $\frac{1}{2}$ nearest-neighbor 2D Heisenberg models on Bravais lattices (square ${ }^{1}$, triangular ${ }^{2}$ ) are Néel ordered at $T=0$. Frustration, small coordination number, competition between interactions can lead to specific quantum phases without magnetic long-range order. Since a decade this subject is a highly debated issue in

aThis generic kind of order, with a macroscopic sublattice magnetization is called in the following magnetic long-range order (LRO), in contrast to other ordered phases where the long-range ordered correlations concern $S=0$ scalar observables (on dimers, quadrumers...) 
the field of quantum magnetism. It was revived by the discovery of high- $T_{c}$ superconductivity in the doped cuprates and fuelled by numerous experimental studies of $2 \mathrm{D}$ antiferromagnetic insulators $\frac{3}{3}$

Section 2 is devoted to the spin- $\frac{1}{2}$ Heisenberg model on the square lattice with first- and second-neighbor interactions $\left(J_{1}-J_{2}\right.$ model). This model is one of the most studied in the field of and this section is a short guide to the literature, with a special emphasis on the various methods used for this problem.

Section 3 deals with general properties of valence-bond crystals (VBC) and related states, the simplest phase which is commonly realized in frustrated spin systems without magnetic LRO.

In section 4 we present large- $N$ generalizations of the Heisenberg model. This approach was extensively developed by Read and Sachdev from the early 90's and has been the first to give an insight into the alternative between VBC and related phases, which have long-range order in local singlet patterns (whence the name of crystals), and resonating valencebond (RVB) spin-liquids (SL) which are translationally invariant phases where the quantum coherence is a central issue.

Section 5 presents some results of quantum dimer models (QDM). These models are effective approaches to the quantum phases of antiferromagnets which are dominated by short-range valence-bonds (or singlets). They have received recently some special attention and provide useful insights onto the phenomenology of VBC and RVB SL.

In Section 6 we review some results concerning models with multiplespin exchange (MSE) (also called ring exchange) interactions. These interactions are now recognized to be present in several physical systems and appear to play an important role in the stabilization of RVB liquid ground states.

The last section is devoted to the Heisenberg model on the kagome lattice (and related models). Despite of an important activity on this subject, the understanding of the low-energy physics of the spin- $\frac{1}{2}$ kagome antiferromagnet remains a challenging problem and we discuss some of the important results and questions.

We should warn the readers that this review is quite "inhomogeneous" and cannot, of course, replace textbooks $\frac{4|5| 6|7|}{4}$ While some parts deal with some rather recent works (QDM or MSE for instance), some others are devoted to older results which we think are still of importance for current research (beginning of the section $J_{1}-J_{2}$, large- $N$ ). The final part devoted to kagome reflects our own views and some unpublished material on still 
unsettled issues. Some parts are intended to be more pedagogical and concrete (QDM and beginning of large- $N$ section) while some others contain more qualitative discussions of the physical issues (end of the section $J_{1}-J_{2}$, VBC, kagome).

\section{2. $J_{1}-J_{2}$ model on the square lattice}

We consider the following Heisenberg model on the square lattice:

$$
\mathcal{H}=2 J_{1} \sum_{\langle i j\rangle} \vec{S}_{i} \cdot \vec{S}_{j}+2 J_{2} \sum_{\langle\langle i j\rangle\rangle} \vec{S}_{i} \cdot \vec{S}_{j}
$$

where $\langle i j\rangle$ and $\langle\langle i j\rangle\rangle$ denote pairs of nearest and next-nearest-neighbors respectively. Although quite simple in appearance, this spin model realizes several interesting phenomena which are relevant to a large class of $2 \mathrm{D}$ frustrated quantum magnets: classical degeneracy, order by disorder, destruction of some long-range order by quantum fluctuations, break down of the spin-wave expansion, opening of a spin gap and (possibly ?) spontaneous translation symmetry breaking, etc. For this reason we start with a general overview of some important results concerning this system. We will focus on the properties related to frustration. A review on the non-frustrated model $\left(J_{2}=0\right)$ can be found in Ref. [1]

\subsection{Classical ground state and spin-wave analysis}

It is easy to find some classical ground state of a translation invariant Heisenberg model on a Bravais lattice because the energy can always be minimized by a planar helix

$$
\vec{S}_{\mathbf{r}}=\vec{e}_{1} \cos (\mathbf{q} \cdot \mathbf{r})+\vec{e}_{2} \sin (\mathbf{q} \cdot \mathbf{r})
$$

provided that the pitch $\mathbf{q}$ minimizes the Fourier transform $J(\mathbf{q})$ of the coupling. $\left[\right.$ In the case of the $J_{1}-J_{2}$ model one has

$$
J(\mathbf{q})=2 J_{1}\left(\cos \left(q_{x}\right)+\cos \left(q_{y}\right)\right)+2 J_{2}\left(\cos \left(q_{x}+q_{y}\right)+\cos \left(q_{x}-q_{y}\right)\right)
$$

- $J_{2}<0.5 J_{1}: J(\mathbf{q})$ has a single minimum at $(\pi, \pi)$. It corresponds to the "usual" Néel state.

- $J_{2}>0.5 J_{1}: J(\mathbf{q})$ has two isolated minima at $(0, \pi)$ and $(\pi, 0)$. They correspond to ferromagnetic lines (resp. columns) arranged in an antiferromagnetic way. These states are sometimes called collinear (in real space). From these planar helix states one can build many 
other ground states by rotating globally all the spins of one sublattice with respect to the other. Although this costs no energy for classical spins at zero temperature, it is known (order by disorder, see below) that configurations where both sublattices have their staggered magnetization collinear in spin space are selected by thermal or quantum fluctuations.

- $J_{2}=0.5 J_{1}: J(\mathbf{q})$ has lines of minima around the edges of the Brillouin zone. At this point the classical ground state is highly degenerate : We can write $\mathcal{H}=\operatorname{cst}+J_{2} \sum\left(S_{1}+S_{2}+S_{3}+S_{4}\right)^{2}$ where the sum runs over all square plaquettes and any state where each plaquette has a vanishing total spin minimizes the classical energy.

Even at the lowest order in $1 / S$, zero-temperature quantum corrections to the sublattice magnetization (order parameter) diverge around $J_{2}=0.5 J_{1}$ (Chandra and Douçot ${ }^{9}$ ). Such large- $S$ approximation usually tends to overestimate the stability of magnetic phases, therefore this breakdown around $J_{2} \sim 0.5 J_{1}$ is a strong evidence for the existence of quantum disordered phase(s) in this region of parameter space.

\subsection{Order by disorder $\left(J_{2}>J_{1} / 2\right)$}

The concept of "order by disorder" was introduced in 1980 by Villain and co-workers $\frac{10}{10}$ in the study of a frustrated Ising model on the square lattice. In this model the next neighbor couplings along the rows are ferromagnetic as well as those on the odd columns (named $A$ in the following). The couplings on the even columns (named $B$ ) are antiferromagnetic. At $T=0$ the ground state has no average magnetization and is disordered. This changes when thermal fluctuations are introduced: a $B$-chain sandwiched between two A chains with parallel spins has lower excitations than a $B$ chain between two $A$-chains with anti-parallel spins. This gives a larger Boltzmann weight to ferrimagnetically ordered states. Villain et al. have exactly shown that the system is indeed ferrimagnetic at low temperature. They were also able to show that site dilution (non-magnetic sites) selects the same ordered pattern, whence the name of "order by disorder".

A somewhat less drastic phenomenon has been observed in quantum systems. It is the selection of particular long-range ordered quantum states among a larger family of ordered solutions which are classically degenerate 
at $T=0$ @ Consider a spin system in which the molecular field created by the spins of one sublattice on the other cancels, which is the case when $J_{2}>0.5 J_{1}$. Shender ${ }^{11}$ showed that if fluctuations are included, the system will select states in which all spins are collinear to each other. This follows from the fact that (moderate) fluctuations at one site are orthogonal to the mean value of the magnetization at that site and the system can gain some magnetic exchange energy by making such fluctuations coplanar on neighboring sites, that is to making the spins collinear. Such a selection of order by quantum fluctuations (and dilution) was discussed by Henley 12 and appears also quite straightly in a spin-wave expansion.13

This selection of the $(\pi, 0)$ or $(0, \pi)$ order spontaneously breaks a fourfold lattice symmetry. An Ising order parameter is thus generated. It takes two values depending whether the ferromagnetic correlations are locally arranged horizontally or vertically. Chandra and co-workers ${ }^{14}$ have studied this mechanism and predicted the existence of a finite temperature Ising phase transition independent of the subsequent development of a sublattice magnetization. This result has been questioned recently $\sqrt{15}$ and the transition has not been observed so far in the spin- $\frac{1}{2}$ model $\left[\frac{16] 15}{10}\right.$ It has however been confirmed by some recent Monte Carlo simulations of the classical Heisenberg model ${ }^{[17}$ Very similar phenomena are present in the $J_{1}-J_{2}$ quantum Heisenberg model on the triangular lattice $\frac{18|19| 20 \mid 21}{12}$

Melzi el $a \stackrel{22 \mid 23}{2}$ have studied a quasi 2D spin- $\frac{1}{2}$ system which is believed to be a $J_{1}-J_{2}$ square lattice Heisenberg antiferromagnet. They found some evidence (splitting of NMR lines) for a collinear $((\pi, 0)$ or $(0, \pi))$ magnetic ordering. Several estimates $\frac{23|24| 25 \mid 16}{1}$ indeed point to $J_{2}>J_{1}$ in this compound.

\subsection{Non-magnetic region $\left(J_{2} \simeq J_{1} / 2\right)$}

Consider the two classical "Ising states" corresponding to the wave vectors $(\pi, \pi)$ and $(\pi, 0)$. They can be taken as (crude) variational states for the Hamiltonian Eq. 1. Their energies (per site) are $E_{\pi, \pi}=-J_{1}+J_{2}$ and $E_{\pi, 0}=-J_{2}$. As discussed above, these states cross at $J_{2}=\frac{1}{2} J_{1}$. However,

\footnotetext{
an Villain's model the system is truly disordered at $T=0$ and an ordered solution is entropically selected at finite temperature. In the quantum $J_{1}-J_{2}$ model above, the classical solutions can adopt various ordered patterns: quantum fluctuations select among these patterns the most ordered one, that is the situation with the highest symmetry and the smallest degeneracy. The ultimate effect of these quantum fluctuations can be the destruction of the Néel order in favor of a fully quantum ground state with $\mathcal{O}(1)$ degeneracy.
} 
one can also consider any first-neighbor singlet (or valence-bond) covering of the lattice as another variational state. In such a completely dimerized state the expectation value of the energy per site is $E_{\text {dimer }}=-\frac{3}{4} J_{1}$, which is below the two Ising states around $J_{2} \simeq J_{1} / 2$. Of course this very simple argument does not prove anything since "dressing" these classical states with quantum fluctuations (spin flips in the Néel-like states or valencebond motions in the dimerized wave functions) will lower the energies of all these trial states and it is absolutely not clear which one may eventually win. Nevertheless, this shows in a simple way why non-magnetic states (i.e rotationally invariant or spin singlet) such as dimerized states can be a route to minimize the energy in a frustrated magnet

\subsubsection{Series expansions}

High-order series expansions can be a powerful technique to investigate frustrated quantum magnets. The general method to generate zero-temperature perturbation expansions in quantum many-body systems was described by Singh et al. ${ }^{[28}$ and Gelfand et al ${ }^{[29}$ For instance, one can consider the following anisotropic model:

$$
\begin{aligned}
\mathcal{H}(\lambda)= & 2 J_{1} \sum_{<i j>}\left[S_{i}^{z} S_{j}^{z}+\lambda\left(S_{i}^{x} S_{j}^{x}+S_{i}^{y} S_{j}^{y}\right)\right] \\
& +2 J_{2} \sum_{<<i j>>}\left[S_{i}^{z} S_{j}^{z}+\lambda\left(S_{i}^{x} S_{j}^{x}+S_{i}^{y} S_{j}^{y}\right)\right]
\end{aligned}
$$

$\mathcal{H}(\lambda=0)$ is a classical Ising model which ground state is known. The series expansion about the Ising limit amounts to compute expectation values in the ground state $|\lambda\rangle$ of $\mathcal{H}(\lambda)$ in powers of $\lambda$ :

$$
\frac{\langle\lambda|\hat{O}| \lambda\rangle}{\langle\lambda \mid \lambda\rangle}=\sum_{n} a_{n} \lambda^{n}
$$

(energy gaps, dispersion relations and susceptibilities can also be computed in the same approach). The calculation of $a_{n}$ requires the enumeration and evaluation of the connected clusters of size $\sim n$, whose number grows exponentially with $n$. Depending on the quantity $\hat{O}$ and on the model, orders from 7 to 20 can typically be obtained on present computers. The series

$\overline{{ }^{\mathrm{b}} \text { Kleir } 26}$ introduced a general procedure to generate local and $S U(2)$ symmetric Hamiltonians for which any first-neighbor dimerized state is an exact ground state. These Hamiltonians are simply defined as sums of projectors which annihilate all dimer coverings. The Majumdar-Gosh 27 chain is the simplest example of a "Klein model". 
is then extrapolated to $\lambda=1$ by standard Padé, Dlog Padé or integrated differential approximations. Such a series expansion about the Ising limit was done by Weihong et al ${ }^{30}$ for the first neighbor square-lattice antiferromagnet. Oitmaa and Weihong ${ }^{31}$ extended the series to the $J_{1}-J_{2}$ model, where each $a_{n}$ is now a polynomial in $J_{1}$ and $J_{2}$. The disappearance of Néel order in the Heisenberg model manifests itself by a vanishing sublattice magnetization as well as some singular behavior of the series for $\lambda_{c}<1$. The results indicate the absence of Néel long-range order in the strongly frustrated region $0.4 \leq J_{2} / J_{1} \leq 0.6$. Such an expansion can locate with a reasonable accuracy the phase boundary of the Néel ordered regions but provides no direct information on the nature of the non-magnetic phase.

To study the model around $J_{2} \simeq J_{1} / 2$, several other expansions have been carried out. A dimer expansion about an exactly dimerized model was done by Gelfand et al. ${ }^{\sqrt[32]{2}}$ Gelfand ${ }^{33}$ Singh et al. ${ }^{[34}$ and Kotov et al. ${ }^{[35} \mathrm{In}$ this approach $J_{1}$ and $J_{2}$ are set to zero everywhere except on isolated bonds arranged in a columnar way and all the other couplings are treated perturbatively. At zeroth order the ground state is simply a product of singlets. In these calculations the dimerized phase remains stable in the intermediate region. Singh et al ${ }^{34}$ also performed a different kind of zero-temperature series expansion. They started from a model of isolated 4-spin plaquettes in order to check a prediction made by Zhitomirsky and Ueda ${ }^{\sqrt{36}}$ that such plaquettes could spontaneously form around $J_{2} \simeq J_{1} / 2$ to produce a state which is invariant under $\pi / 4$ lattice rotations. Although the ground state energy they obtained is very close to the one obtained from the dimerized limit (within error bars of the extrapolation procedure) they observed an instability in the plaquette scenario (the triplet gap vanishes before reaching the isotropic square-lattice model) which suggests that plaquette order is not the issue (the analysis of the exact numerical spectra for 36 sites confirmed this result $\left.{ }^{37}\right)$.

Sushkov et al ${ }^{38}$ (improved numerical results compared to Ref. ${ }^{39}$ ) computed the susceptibility $\chi_{D}$ associated with the appearance of columnar dimer order in the $(\pi, \pi)$ Néel phase by a series expansion about the Ising limit. Such a susceptibility seems to diverge at $J_{2} / J_{1}=g_{c 1} \simeq 0.405 \pm 0.04$. On the other hand the disappearance of the magnetic LRO is observed (through the Néel order parameter or through the anisotropy in spin space of the spin-spin correlations) at $J_{2} / J_{1}=g_{c 2} \simeq 0.39 \pm 0.02$. This point could a priori be different from $g_{c 1}$. In such a case the system would first break the $\pi / 4$ lattice rotation symmetry at $g_{c 1}$, while magnetic LRO remains (gapless spin waves). Only at $g_{c 2}>g_{c 1}$ the $S U(2)$ rotation symmetry is restored and 
the magnetic excitations acquire a gap. From field theoretical arguments based on effective actions valid close to the critical points, Sushkov et al ${ }^{38}$ argue that the proximity (or possible equality) of $g_{c 1}$ and $g_{c 2}$ is a general feature in frustrated magnets which originate from the coupling of triplet and singlet excitations.

Sushkov et al ${ }^{39}$ computed susceptibility $\chi_{P}$ associated to plaquette order by an expansion around the dimerized limit, assuming that the system has columnar dimer LRO. The result shows a divergence of $\chi_{P}$ when $J_{2} / J_{1} \rightarrow g_{c 3}=0.5 \pm 0.02$. From these results Sushkov et al. suggested that the translation symmetry along the columns is broken down at $g_{c 3}$ (giving rise to an eight-fold degenerate ground state in the thermodynamic limit) before the $(\pi, 0)-(0, \pi)$ magnetically ordered phase appears at $g_{c 4} \simeq 0.6$. This picture is qualitatively consistent with the spin-spin correlations computed in a $10 \times 10$ system with a density matrix renormalization group (DMRG) algorithm. $\cdot \underline{40}$

Due to the relatively short series (typically of order 7) involved and the uncertainties in the extrapolation procedures, such results should be confirmed by other methods but this succession of quantum phase transitions represents a very interesting scenario. We note that if the model has a fully symmetric liquid ground state in some parameter range, it should be difficult to capture from series expansions about limits where some lattice symmetries are explicitly broken.

\subsubsection{Exact diagonalizations}

Exact diagonalizations have a priori no bias, and were used very early in this field $\underline{41 / 42 / 43}$ Large-size computations and sophisticated finite size scaling analysis are nevertheless needed to extract significant information. Schulz et al. ${ }^{[4}$ performed extensive exact diagonalizations of the $J_{1}-J_{2}$ model for system sizes up to 36 sites. They analyzed the behavior of several quantities such as structure factors (Néel order parameter), ground state energy, spinwave velocities (obtained from the finite size corrections to the ground state energy), spin stiffness and uniform susceptibility. Their analysis, including quantitative comparisons with non-linear sigma model predictions, $\frac{45}{4}$ concluded to the absence of Néel long-range order in the strongly frustrated region $0.4 \leq J_{2} / J_{1} \leq 0.6$. There, they show enhanced columnar dimerdimer correlations as well as chiral ones but the size effects were not clear enough to discriminate between short or long-range order for these order parameters. 


\subsubsection{Quantum Monte Carlo}

Quantum Monte Carlo (QMC) methods have been extensively applied to the $J_{1}-J_{2}$ model in the low frustration regime giving an highly accurate description of the Néel phase (Sandvik $\frac{\sqrt{46}}{4}$ and Refs. therein). In the nonmagnetic and highly frustrated regime a simple QMC approach is ineffective due to the so-called sign problem. The fixed node approach is the first answer to this problem: the exact imaginary time propagator $e^{-\tau \mathcal{H}}$ used to filter out the ground state from a variational guess $\left|\psi_{g}\right\rangle$ is replaced by an approximate propagator, which has the same nodes as $\left|\psi_{g}\right\rangle$. The quality of the result depends on the quality of the nodal regions of $\left|\psi_{g}\right\rangle$. Various schemes have been used to try to go beyond this limitation: stochastic reconfiguration (Sorella $a^{47}$ ), eventually associated to a few Lanczos iterations $\left.48\right|^{37}$ An alternative method has been devised by du Croo de Jongh et al., $\frac{40}{\text { where }}$ the guiding function is replaced by the result of a DMRG calculation 4955 Both methods have their own bias. Using the first of them, Capriotti and Sorella $\sqrt{37}$ concluded that for $J_{2} / J_{1} \sim 0.45$ a Gutzwiller-projected BCS wave function $|p B C S\rangle$ was an excellent guiding wave function:

$$
\begin{aligned}
|p B C S\rangle & =\hat{\Pi}|B C S\rangle \\
|B C S\rangle & =\exp \left(\sum_{i, j} f_{i, j} c_{i \uparrow}^{\dagger} c \dagger_{j \downarrow}\right)|0\rangle
\end{aligned}
$$

where $|0\rangle$ is the fermion vacuum, $c_{i \uparrow}^{\dagger} c \dagger_{j \downarrow}$ creates a valence-bond on sites $(i, j)$ and $\hat{\Pi}$ projects out states with double occupancy. The pairing amplitude $f_{i, j}$ (often called gap function $\Delta_{k}$ ) ${ }^{\mathrm{C}}$ is optimized with a Monte Carlo algorithm in order to minimize the energy. Capriotti and Sorella gave convincing indications that their wave function is quite accurate. The best variational energies are obtained in the frustrated region with a pairing amplitude which mixes $d_{x^{2}-y^{2}}$ and $d_{x y}$ symmetries. In particular it reproduces the correct nodal structure of the ground state in the frustrated region at least for moderate system sizes where the variational result can be checked against the exact result. This is a subtle and non-trivial information for systems which do not obey the Marshall's sign rule as this frustrated model. They concluded from these results that the system probably had no longrange order neither in dimer-dimer correlations nor in four-spin plaquette correlations. On the other hand, du Croo de Jongh et al. argued in favor

$\overline{\mathrm{c}}_{\mathrm{c} \text { After the Gutzwiller projection } \Delta_{k}}$ is no longer the observable gap 51 
of columnar dimerized phase which also break the translation symmetry along the columns (plaquette-like correlations similar to those found by series expansions $\sqrt{39})$.

The comparison of the results of these different approaches shows that this problem remains a very challenging one. The model in the frustrated regime is probably never very far from a quantum critical point and in these conditions none of the available methods seems able to discriminate between a VBC with tiny gaps both in the singlet and triplet sectors, a critical phase with a quasi order in dimers and gapless singlet excitations, or a true SL with gaps in any sector of spin but no long-range order in any observable. As we will explain in the following sections some other frustrated models are happily deeper in the non-magnetic phases and exhibit quantum phases which are easier to characterize.

\section{Valence-bond crystals}

\subsection{Definitions}

Among the different quantum solutions to overcome frustration the VBC is the simplest scenario. In this phase, neighboring spins arrange themselves in a regular pattern of singlets: dimers quadrumers or $2 n$-mers $S=0$ plaquettes. The stability of this phase comes from the extreme stability of small $S=0$ clusters (recall that the energy of a singlet of two spins $\frac{1}{2}$ is $-3 / 4$ to be compared to the energy of two classical (or Ising) spins which is only $-1 / 4$ ), and eventually from the fact that frustrated bonds between two different singlets do not contribute to the total energy.

In a VBC phase there is no $S U(2)$ symmetry breaking, no long-range order in spin-spin correlations, but long-range order in dimer-dimer or larger singlet units. Except at a quantum critical point, all excitations of a VBC are gapped. Depending on the lattice geometry, such a wave function can spontaneously break some lattice symmetry (spontaneous $V B C$ ) or can remain fully symmetric (explicit $V B C$ ). In a strict sense, the name VBC should be reserved for systems with a spontaneous lattice symmetry breaking. However, since these two kinds of systems share many similarities we will discuss both in this section.

When the Hamiltonian has some inequivalent bonds and an integer spin in the unit cell (even number of spin- $\frac{1}{2}$ for instance) the system can take full advantage of the strong bonds and minimize the effects of the frustrating

\footnotetext{
${ }^{\mathrm{a}}$ whence the name Spin Peierls phase sometimes given to the VBC phase.
} 
ones. In that case the explicit VBC is the "natural" strong coupling solution. One can build a simple Hamiltonian in which the bonds which are not occupied by the singlet objects are turned off. The resulting model is a set of small decoupled clusters (dimers or larger plaquettes) and the ground state is a trivial product of singlets. Importantly, this strong coupling limit has the same lattice symmetry as the original one. Going back to the original Hamiltonian no quantum phase transition is encountered when going from the trivial singlet product up to real interacting ground state. Models with an half-odd-integer spin in the unit cell cannot realize a VBC unless they spontaneously enlarge their unit cell. In these situations there is no unique elected position for the $2 n$-mers and a symmetry breaking must take place in order to form a VBC. Examples of these two kinds of VBC will be given below.

\subsection{One-dimensional and quasi one-dimensional examples (spin- $\frac{1}{2}$ systems)}

One of the simplest example of (spontaneous) VBC is observed in the $J_{1}-J_{2}$ model on the chain for $J_{2} / J_{1}>\left(J_{2} / J_{1}\right)_{c} \sim 0.24$ 27|52|53|54|55 For $J_{2} / J_{1}=$ 0.5 the doubly degenerate ground states are exact products of dimers $27 / 5$

$$
\left.\left|M G_{ \pm}>=\prod_{n=1}^{N / 2}\right|(2 n, 2 n \pm 1)\right\rangle .
$$

Here and in the following we call "dimer" a pair of spins in a singlet state, and note it:

$$
\left|(i, j)>=\frac{1}{\sqrt{2}}[|i,+\rangle|j,-\rangle-|i,-\rangle|j,+\rangle] .\right.
$$

For all $J_{2} / J_{1}>\left(J_{2} / J_{1}\right)_{c}$, the ground states are products of dimers, dressed by fluctuations of valence bonds, dimer long-range order persists in all the range of parameters. This model has gapful excitations which can be described as pairs of scattering spin- $\frac{1}{2}$ solitons separating the two exact ground states 5 (these fractionalized excitations are specific of the 1D chain).

The Heisenberg chain with alternating strong and weak bonds (Spin Peierls instability), has indeed a unique ground state where the dimers are mainly located on the strong bonds. In that case, the spin- $\frac{1}{2}$ excitations are confined by the underlying potential and the true excitations are gapful integer magnons ( $56 \mid 57$ and refs. therein). It is an explicit VBC. 
A two-leg ladder with AF rung exchange has also a unique VBC ground state and gapped magnons as excitations. $\frac{58}{}$ On the other hand Nersesyan and Tsvelik $k^{59 \mid 60}$ have proposed an example of frustrated ladder, with a spontaneously dimerized ground state, and gapful excitations. Excitations of this last model are identified as pairs of singlet and triplet domain walls connecting the two ground states, they form a continuum.

As can be seen from this rapid and non exhaustive enumeration, VBC ground states are relatively frequent in frustrated one-dimensional spin$\frac{1}{2}$ models. All these systems are gapful but the excitations could be of different nature emerging as modes (associated to integer spin excitations) or continuum of pairs of excitations that could be fractionalized (it is then specific of one-dimensional systems) or not.

\subsection{Valence Bond Solids}

The VBS wave function was introduced by Affleck, Kennedy, Lieb and Tasaki (AKLT) $\frac{6162}{16}$ It can be constructed whenever the spin $S$ on a site is a multiple of one half the coordination number $z: 2 S=0 \bmod z$. Let us consider the simplest case $2 S=z$. In that case the local spin $S$ can be seen as the symmetric combination of $2 S$ (fictitious) spin- $\frac{1}{2}$. Now on each bond of the lattice one can make a singlet between two fictitious spins- $\frac{1}{2}$. Such a product of singlets does not belong to the physical Hilbert space of the original spin- $S$ model but to a much larger space. The VBS wave function is defined as the projection of the singlet-product state onto the physical space. This projection amounts to symmetrize (for all lattice sites) the wave function with respect to the fictitious spins to force them into a physical spin- $S$ state. A VBS can be viewed as an explicit $V B C$ of the fictitious spins. Simple Hamiltonians with short-range and $S U(2)$-symmetric interactions for which the VBS is an exact ground state can be constructed (sum of projectors 5162$)$. By construction the VBS wave function is a spin singlet and breaks no lattice symmetry. By extension we may say that a system is in a VBS phase if its ground state can be adiabatically transformed into the VBS wave function without crossing a phase transition. As the VBC, models in the VBS phase have a gap to all excitations but their wave functions are slightly more complex and their order parameter is non-local. The order of VBS is associated to long-range singlet-singlet correlations in

\footnotetext{
bhis may however not always be true when the coordination number of the lattice is large ${ }^{61}$ In such cases the VBS wave function is still a spin singlet but has long-range spin-spin correlations. We do not consider such cases here.
} 
the fictitious spins. Expressing such observable in terms of the physical spins leads to a non-local quantity called string order parameter $63[64$ Contrary to explicit VBC, VBS have fractionalized degrees of freedom at the edges of the system with open boundary conditions. These are simply associated to the unpaired fictitious spins. To our knowledge these properties have not been explored in quantum 2D systems.

The spin-1 Heisenberg chain is the prototype of VBS in 1D Such a state has a unique ground state, a gap in the excitations and exponentially decreasing spin-spin and dimer-dimer ${ }^{65}$ correlations. See the chapter by P. Lecheminant in this volume for more details about the VBS phase of the spin-1 chain.

A spin- $\frac{3}{2}$ specific $S U(2)$-invariant model on the honeycomb lattic $\frac{61 \mid 62}{6}$ is another example of 2D VBS. The spin-1 Heisenberg model on the kagome lattice was proposed to realize a VBS-like ground state ${ }^{\sqrt{66}}$ in which singlets form on every hexagon without any spontaneous symmetry breaking (hexagonal singlet solid) d A similar approach was carried out for the spin-1 pyrochlore Heisenberg model ${ }^{67}$ In that case a lattice distortion was invoked to lift the degeneracy between the two singlet states of each tetrahedron.

\subsection{Two-dimensional examples of $V B C$}

\subsubsection{Without spontaneous lattice symmetry breaking}

Two spin- $\frac{1}{2}$ experimental examples of $2 \mathrm{D}$ (explicit) VBC have recently attracted attention: $\mathrm{CaV}_{4} \mathrm{O}_{9} \sqrt{68|69| 70|71| 72|73| 74}$ and $\mathrm{SrCu}_{2}\left(\mathrm{BO}_{3}\right)_{2} \frac{75|76 / 77 / 78| 79|80| 81|82| 83 \mid 84}{} \mathrm{In} \mathrm{CaV}_{4} \mathrm{O}_{9}$ the $\mathrm{V}^{4+}$ ions are on a $1 / 5$ depleted square lattice and in $\mathrm{SrCu}_{2}\left(\mathrm{BO}_{3}\right)_{2}$ the exchange couplings between $\mathrm{Cn}^{2+}$ ions realize the Shastry Sutherland model. ${ }^{85}$ A lattice embedding the main couplings of these two physical problems is drawn in Fig. 1 Interactions are of the Heisenberg type e

VBC are obvious ground states in the strong coupling limits of each problem.

The exact ground state of the Shastry Sutherland model is built from singlets on the $J^{\prime}$ bonds $\stackrel{85 \mid 77]}{\text { For }} J^{\prime} / J \gtrsim 1.55 \pm 0.05$ the model has a

\footnotetext{
"In 1D, some authors call "Haldane systems" all the spin-gapped phases, whatever their true nature: VBC or VBS.

${ }^{\mathrm{d}}$ Each kagome site belongs to two hexagons. Each physical spin-1 can be split into two spin- $\frac{1}{2}$, each of them being involved in the formation of a singlet on one neighboring hexagon.

eSmall Dzyaloshinsky-Moriya interactions have been identified in $\mathrm{SrCu}_{2}\left(\mathrm{BO}_{3}\right)_{2}$.
} 


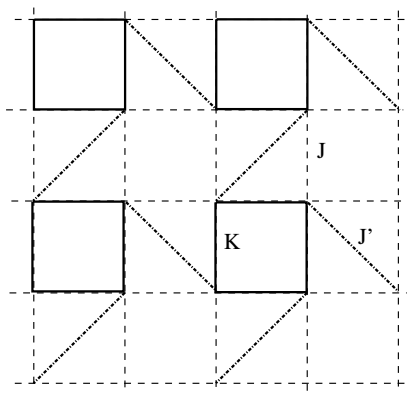

Fig. 1. The $\frac{1}{5}$-depleted lattice and the Shastry Sutherland lattice. The strong bonds of the Shastry Sutherland model are the bonds $J^{\prime}$ (dotted dashed lines): they can accommodate orthogonal dimers $(J=K$ can be considered as a "perturbation" in the real $\left.\mathrm{SrCu}_{2}\left(\mathrm{BO}_{3}\right)_{2}\right)$. The lattice formed by the strongest exchanges in $\mathrm{CaV}_{4} \mathrm{O}_{9}$ is obtained with $J=0$. The phase diagram of this mode ${ }^{82}$ contains (at least) collinear Néel phases, dimer and 4-spin plaquette VBC.

gap in the spectrum of excitations and is in a dimerized VBC phase. $f \mathrm{f}$ For $J^{\prime} / J \lesssim 1.15 \pm 0.05$ the system is in the $(\pi, \pi)$ Néel state of the square lattice (results of zero-temperature series expansion $[87$ ). The possibility of an intermediate phase, possibly with helical short-range correlations, has been actively discussed in the literature $\$ 88 / 7989 \mathrm{~S}$. Miyahara and K. Ueda have recently written a review of the theory of the orthogonal dimer Heisenberg spin model for $\mathrm{SrCu}_{2}\left(\mathrm{BO}_{3}\right)_{2}$. 84

The $\frac{1}{5}$-depleted Heisenberg square lattice model $(J=0)$ has been studied as a function of the ratio of the two different couplings: bonds within a plaquette $(K)$ and dimer bonds $\left(J^{\prime}\right)$ between plaquettes. At isotropic coupling $\left(J^{\prime}=K\right)$ collinear long-range Néel order survives the depletion (the order parameter is about $35 \% \frac{71}{71}$ of the maximum classical value). A small unbalance in couplings drives the system either in a 4-spin plaquette $\operatorname{VBC}\left(K>J^{\prime}\right)$ or in a dimer VBC $\left(K<J^{\prime}\right)$. Both (explicit) VBC phases have a spin gap. A recent generalization of these models by Läuchli et al encompasses both the $\frac{1}{5}$-depleted Heisenberg square lattice model and the Shastry Sutherland mode ${ }^{82}$ (see Fig. 1). Its phase diagram exhibits collinear Néel phases $((\pi, \pi)$ or $(0, \pi))$ separated from the VBC phases by

${ }^{\mathrm{f}}$ Consider a decomposition of the Shastry-Sutherland lattice as edge- and corner-sharing triangles. For $J^{\prime}=2 J$ the Hamiltonian is accordingly written as a sum of $J\left(\vec{S}_{1}+\vec{S}_{2}+\vec{S}_{3}\right)^{2}$ terms for each triangle ( $J^{\prime}$-bonds are shared by two triangles) and each such term is minimized by the dimerized state. This shows rigorously that the dimerized state is the ground state as soon as $J^{\prime} / J \gtrsim 2$. 
second order phase transitions. Transition between the two VBC phases which have different symmetries occurs via a first order phase transition

Excitations in these models come from the promotion of local singlets to triplet excitations. In 2D the ordered dimer background provides a confining force for the spin- $\frac{1}{2}$ excitations. Indeed, separating two unpaired spins (that is two spinons) creates disruption in the ordered pattern all the way from the first to the second. The energy cost is thus proportional to the length of the string of defaults and both spin- $\frac{1}{2}$ excitations remains in fact confined. Only integer spin excitations are expected. On the other hand in these strongly coupled models single-triplet hoppings can be difficult and correlated motions might be important, leading to a large zoology of excited modes (see Ref. 11 and references therein). This potential frustration of the triplet motion favors the appearance of magnetization plateaus in

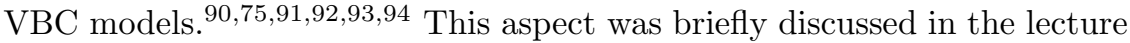
notes published by the authors $\frac{95}{5}$

\subsubsection{With spontaneous lattice symmetry breaking}

In the previous models the (explicit) VBC phases do not break any lattice symmetry. They can be directly related to the geometry and relative strength of the couplings. In more symmetric situations with frustration, spontaneously symmetry breaking VBC can appear as a way to overcome this frustration by taking full advantage of the quantum fluctuations. This is probably the case in the $J_{1}-J_{3}$ model on the square lattice for intermediate $J_{3} / J_{1} \sim 0.6, \frac{96}{9}$ in the $J_{1}-J_{2}$ model on the hexagonal lattice for intermediate $J_{2} / J_{1} \sim 0.4,97$ and in the Heisenberg model on the checker-

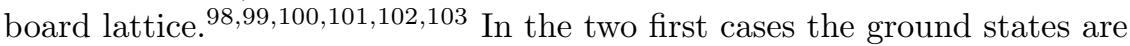
dressed columnar VBC of dimers. Translation and $C_{4}$ (resp. $C_{3}$ only) symmetries of the lattice are spontaneously broken. The ground state is 4 (resp. 3) times degenerate. Spin-spin correlations decrease exponentially with the system size. All excitations are gapped. Contrary to the $J_{1}-J_{2}$ model on the square lattice, exact diagonalization $\$ 96|97| 99$ give a rather straightforward information on these systems where the correlation lengths are small enough (far enough from the critical points which limit the boundary of the VBC phases).

The spin- $\frac{1}{2}$ Heisenberg model on the checker board lattice, which

g A recent $S p(N)$ study of the Shastry Sutherland mode 89 suggests that a spin liquid phase with deconfined spinons might appear in such a model. No evidence of such a phase has emerged from the $S U(2)$ studies so far. 
can also be seen as a planar lattice of corner sharing tetrahedrons (see Fig. 2), has received the largest attention for different reasons \begin{tabular}{|l|l|l|l|l|l|l|l|}
104 & 105 & 98 & 101 & 100 & 99 & 102 & 103
\end{tabular} The problem has classically a continuous
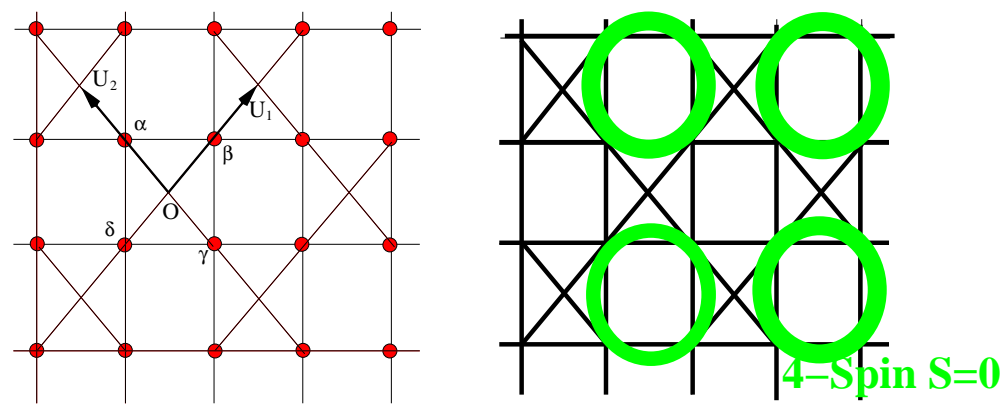

Fig. 2. Left: the checkerboard lattice. The spins sit at the vertices shown by bullets, all couplings are identical, $\mathbf{u}_{1}, \mathbf{u}_{2}$ are the unit vectors of the Bravais lattice. Right: the ground states of the Heisenberg model on the checker board lattice are translational symmetry breaking VBC of 4-spin plaquettes on the uncrossed squares.

local degeneracy: the Hamiltonian can be rewritten as the sum of the squares of the total spin of each tetrahedron, and every configuration with a zero spin on each tetrahedron is a ground state. Classically this problem shares this property with the Heisenberg model on the kagome, pyrochlore $\stackrel{106 \mid 107}{\longrightarrow}$ garnet $\stackrel{108}{~ a n d ~ p y r o c h l o r e ~ s l a b ~} \frac{109}{1 a t t i c e s}$ (these lattices made of corner sharing "simplexes" with 2, 3 or 4 spins each were dubbed "bisimplex" lattices by Henley 1100 ).

The quantum spin- $\frac{1}{2}$ antiferromagnet on the kagome lattice has been found to be quite specific with a small gap (if any) toward triplet excitations and an anomalous density of gapless low-lying singlets excitations (see 77 .

The quantum scenario on the checkerboard lattice is quite different. The ground state is a (dressed) product of 4 -spin $S=0$ plaquettes on uncrossed squares: this state breaks translational symmetry but not $C_{4}$ (the point group being defined at the center of an empty plaquette). It has a two fold degeneracy in the thermodynamic limit: this is easily seen in the symmetries and finite size scaling of the low lying levels of exact spectra. ${ }^{99}$ The choice of the 4-spin $S=0$ states on the uncrossed squares corresponds to the most stable local configuration. The product of $S=0$ quadrumers is only weakly renormalized in the exact ground state. ${ }^{99}$

Excitations of this model have been studied in different approaches, 
exact diagonalizations 111 , series expansions, $\frac{101}{1}$ real space renormalization transformation $[102$ All the excitations (singlet and triplet) are gapped. The triplet excitations originate from the triplet excitation of an uncrossed plaquette, they are weakly dispersive with a large gap. The singlet excitations cannot be described so simply: from exact diagonalizations data one can suspect that some of these excitations come from the reorganization of two adjacent triplet on crossed squares. The Contractor Renormalization (CORE) method of Berg et al ${ }^{[102}$ on the other hand suggests that these excitations are domain walls between the two translated plaquette-VBC ground states.

\subsection{Methods}

Spin waves ${ }^{69}$ and Schwinger bosons $\$ 88770$ are simple approximations to study the phase diagram of a quantum frustrated magnet. But the first method only gives an approximate knowledge on the range of existence of the Néel phases, and it is rather difficult to include the effect of fluctuations beyond the mean-field approximation within the Schwinger-Boson formulation. As discussed in the section devoted to large- $N$ approaches ( $\$ 4$, spontaneous lattice symmetry breaking are very likely to be due to topological effects (Berry phase of instantons) which cannot be captured by the mean-field state. This probably explains why no spontaneous VBC has ever been found (to our knowledge) in Schwinger-Boson calculations ${ }^{\mathrm{h}}$ For unfrustrated problems, as the Heisenberg model on the $\frac{1}{5}$-depleted square lattice ${ }^{71} \mathrm{QMC}$ is considered as the method which can give benchmarks for other approaches.

Although VBC are naturally described with the help of spin- $\frac{1}{2}$ valencebonds, the appearance of such states in the low-spin limit can sometimes be anticipated from an appropriate large- $S$ approach. An example is given in the work of Tchernyshyov et al ${ }^{[103}$ (see also a previous paper by Henley $\stackrel{110}{ }$ ) on the checkerboard Heisenberg model. In this model, when both couplings (square lattice bonds and diagonal bonds) are equal, ground state has a continuous local degeneracy. However leading $1 / S$ corrections select collinear states out of this huge manifold $\frac{110}{10}$ There remains an exponential number of such states and they do not have any magnetic order. However they exhibit long-range bond order and a spontaneous symmetry breaking ${ }^{103}$ which

\footnotetext{
${ }^{\mathrm{h}}$ It seems however that spontaneous $\mathrm{VBC}$ naturally arise in large- $N$ approaches with fermionic representation of $S U(N)$ when $1 / N$ corrections are considered (see section 5.2 .
} 
is analogous to the one observed numerically in the spin- $\frac{1}{2}$ case.

For frustrated problems, exact diagonalizations can be useful tools in situations where the system is not too close from a critical point, that is when the correlations length is not too large. Successful applications of exact diagonalizations methods to 2D Heisenberg magnets realizing a VBC include studies of the $J_{1}-J_{3}$ model on the square lattice, ${ }^{96} J_{1}-J_{2}$ model on the hexagonal lattice ${ }^{97}$ Heisenberg model on the checkerboard lattice ${ }^{99}$ In such situations analysis of the quasi degeneracy of the low-lying levels of the spectra and of their finite-size scaling gives an unbiased and direct informations on the symmetry breakings in the thermodynamic limit ${ }^{99}$ However the boundaries of the phases and the quantum critical points cannot be accurately determined with this method. The series expansions described in the previous section appears to be a powerful approach to determine phase boundaries 112744 If the spin-spin correlation length is not very short, as it is the case in the $J_{1}-J_{2}$ model on the square lattice for $J_{2} / J_{1} \sim 0.5, \underline{37}$ it is very difficult to decide from exact diagonalizations between a VBC, a critical phase or an RVB SL.

Concerning excitations, exact diagonalizations give the gaps in each sector and provide a crude approximation of the dispersion laws of the first excitations. The large-scale nature of the excitations (as for examples domain walls excitations) can escape this method. The semi-analytical approaches which can be used for the study of the excitations of the VBC, all use as a basic departure point the excitations of a local cluster of spins conveniently renormalized by perturbation ${ }^{73 \mid 101}$ or effective renormalization technique. ${ }^{102}$ Contrary to exact diagonalizations these methods are not limited by finite-size effects but the results can be biased by the departure point $101 \mid 102$

\subsection{Summary of the properties of VBC phases}

The generic features of $\mathrm{VBC}$ (whatever the dimensionality of the lattice) are:

- A spin gap, no $S U(2)$ symmetry breaking and short-range spin-spin correlations,

- Long-range order in dimer-dimer and/or larger $S=0$ plaquettes. The coupling of this order to lattice distortions is probable in experimental realizations of spontaneous VBC.

- In spontaneous VBC phases the ground state is degenerate. From the theoretical point of view the discrete symmetry of the order 
parameter of the VBC which spontaneously breaks a lattice symmetry may give birth to a finite temperature Ising-like transition. $\frac{14}{14}$ Simultaneity between this transition and a possible structural transition is likely when the couplings of the spins to the lattice degrees of freedom (phonons) is considered 113

- VBC have gapped excitations, in the $S=0$ sector as well as in other $S$ sectors. A wide zoology of modes is to be expected as well as continua associated to multi-particle excitations or scattering of domain walls (in the case of a spontaneous symmetry breaking of the ground state). In two dimensions all these excitations have integer spins (the ordered back-ground inducing a confinement of the spin- $\frac{1}{2}$ excitations)

Frustration on the square lattice or more generally on bipartite lattices is often overcome by VBC phases. The appearance of VBC in triangular geometries is possible in principle but there is up to now no examples of such phases in pure spin- $\frac{1}{2}$ models (in Sec. 5 examples will be given within the framework of quantum dimer models).

It has been advocated in the large- $N$ approaches (see section (4) that, at least in two dimensions, collinear spin-spin correlations generically lead to VBC or VBS and only non-collinear spin-spin correlations can give birth to RVB SL with unconfined spin- $\frac{1}{2}$ excitations. The present knowledge of $S U(2)$ phase diagrams supports this prediction. The VBC found so far numerically in $S U(2)$ spin models appear to be in regions of parameter space where the spin-spin correlations are characterized by some shortrange collinear order in the large- $S$ limit. The $J_{1}-J_{2}$ model on the honeycomb lattice has a classical incommensurate phase in the regime of high frustration and there are some evidences that in the quantum phase diagram the collinear phase is separated from the columnar VBC phase by a RVB SL 97 The multiple-spin exchange (MSE) model on the triangular lattice is also believed to be a RVB SL ${ }^{114}$ and the corresponding classical ground states generically have non-coplanar spin configurations. Capriotti et al ${ }^{37}$ argued that the spin- $\frac{1}{2}$ square lattice $J_{1}-J_{2}$ model could be a RVB SL. If confirmed, this would be the first counter-example to the general rule explained above (The Heisenberg model on the pyrochlore lattice might be an other counter-example $\left.e^{102}\right)$. 


\section{Large- $N$ methods}

Introduced by Affleck, $\frac{115}{11}$ Affleck and Marston, $\frac{116}{1}$ Arovas and Auerbach $\frac{117}{117}$ and Read and Sachdev $\sqrt{118|119| 120}$ in the context of spin models, large- $N$ approaches are powerful methods to investigate quantum antiferromagnets. When $N$ is taken to infinity many of these models can be solved by saddle point methods and finite- $N$ corrections can be, at least in principle, explored in a controlled way. A success of these approaches is that they can describe the phenomenology of a large variety of phases encountered in quantum magnets : Magnetic LRO (possibly with order by disorder selection) as well as phases dominated by short-range valence-bonds: VBC, VBS and RVB liquids. One crucial result (due to Read and Sachdev $118|119| 120|121| 122 \mid$ ) concerning these three later phases is that the analysis of finite- $N$ corrections to some large- $N$ formulations ( $S p(N)$ for instance, see below) provides a general criterion to decide which of these three phase should appear in a given model.

This criterion is the following in 2D: if the (large- $N$ equivalent of the) "spin" $S$ at each site matches the lattice coordination number $z$ by $2 S=0 \bmod z$ a VBS is to be expected. If it is not the case (as for a spin- $\frac{1}{2}$ model on the square lattice) one should look at the local spin-spin correlations. If they are reminiscent of a collinear order, a VBC with spontaneous translation symmetry breaking is expected whereas non-collinear short-range correlation generically give rise to a RVB phase without any broken symmetry and deconfined spinon excitations. These results are of course based on a large- $N$ generalization of the original spin model and there is no guaranty at all that these rules should always apply to $S U(2)$ models. To our knowledge they have however not been manifestly found in error up to now. In the following we will present some of the important reasoning steps leading to this result.

\subsection{Bond variables}

The $S U(2)$ algebra of a spin $S$ at one site can be represented by 2 species of particles $a_{\sigma}^{\dagger}$ (with $\sigma=\uparrow, \downarrow$ ), provided that the total number of particles on one site is constrained to be $a_{\uparrow}^{\dagger} a_{\uparrow}+a_{\downarrow}^{\dagger} a_{\downarrow}=2 S$. The raising operator $S^{+}$ (resp. $S^{-}$) is simply represented by $a_{\uparrow}^{\dagger} a_{\downarrow}$ (resp. $a_{\downarrow}^{\dagger} a_{\uparrow}$ ). These particles can be chosen to be fermions (Abrikosov fermions) or bosons (Schwinger bosons). These particles carry a magnetization $\pm \frac{1}{2}$ since $S^{z}=\frac{1}{2}\left(a_{\uparrow}^{\dagger} a_{\uparrow}-a_{\downarrow}^{\dagger} a_{\downarrow}\right)$. For this reason they are often called spinons. The Heisenberg interaction is a 
quartic interaction for these particles:

$$
\vec{S}_{i} \cdot \vec{S}_{j}=S^{2}-\frac{1}{2} A_{i j}^{\dagger} A_{i j}
$$

with the bond operator $A_{i j}$ defined by:

$$
A_{i j}=a_{j \downarrow} a_{i \uparrow}-a_{j \uparrow} a_{i \downarrow}
$$

Acting on the vacuum, $A_{i j}^{\dagger}$ creates a spin singlet on the bond $(i j)$. Physically $A_{i j}^{\dagger} A_{i j}$ measures the number of singlets on that bond and Eq. 10 shows that the antiferromagnetic Heisenberg interaction just tries to maximize that number.

The idea of large- $N$ methods is to generalize the $S U(2)$ symmetry of the spin-S algebra to a larger group $S U(N)$ (or $S p(N)$ ) by letting the index $\sigma$ go from 1 to $N$ (or $2 N$ ). The $S U(N)$ (or $S p(N)$ ) generalization of the Heisenberg model is solved by a saddle point calculation of the action. The $N=\infty$ limit is very similar to a mean-field decoupling of the four-body interaction of the physical $S U(2)$ model: $A_{i j}^{\dagger} A_{i j} \simeq A_{i j}^{\dagger}\left\langle A_{i j}\right\rangle+\left\langle A_{i j}^{\dagger}\right\rangle A_{i j}-$ $\left|\left\langle A_{i j}\right\rangle\right|^{2}$.

\section{2. $S U(N)$}

The generalization of the Heisenberg model to such a symmetry group depends only on the choice of the irreducible representation of $S U(N)$ according to which the "spin" operators transform (and not on the choice of fermions or bosons to implement the representation). For $S U(2)$ this amounts to specify the magnitude $S$ of the spin. For $S U(N)$ irreducible representations are labeled by Young tableaux. The case of a general rectangular tableau with $n_{c}$ columns and $m$ rows was discussed by Read and Sachde $\sqrt{123}$ and $n_{c}$ appears to continue to play a role similar to $2 S$ in the large- $N$ phase diagrams ${ }^{a}$ In this review we will focus on a slightly different large- $N$ generalization of the $S U(2)$ model which is both able to deal with frustration and magnetic states.

a Taking the limit $N \rightarrow \infty$ with $m$ fixed of order 1 and $n_{c} \sim N$ is most conveniently done with bosons $b_{\alpha p}^{\dagger}$ where $\alpha=1 \cdots N$ is the $S U(N)$ index, while $p=1 \cdots m$ label the different "colors". There are therefore $N m$ kinds of bosons. On the other hand it is convenient to use $n_{c}$ "colors" of fermions (still with an $S U(N)$ index) to deal with the case $n_{c} \sim \mathcal{O}(1)$ and $m \sim N$. Bosonic representations with $n_{c} \sim N$ are appropriate to describe magnetically ordered phases $\frac{117}{17}$ but cannot be used for frustrated models (such a representation is not self-conjugate). On the other hand fermionic representations, such as the $m=N / 2$ and $n_{c}=1$ one $116 \mid 124$ can be used on any lattice but they do not display magnetically ordered phases and tend to favor dimerized states $116|123| 124$ 


\section{3. $\operatorname{Sp}(N)$}

To perform a large- $N$ extension of frustrated Heisenberg models one has to use either fermions $\frac{116}{16}$ or bosons with an $S p(N)$ symmetry. The latter seems to produce phase diagrams that closely resemble the $S U(2)$ problems and we will focus on this representation which was introduced by Read and Sachdev ${ }^{120}$ The presentation below is largely inspired from their papers $118|119| 120|121| 122$

We now have $2 N$ flavors of bosons at each site: $b_{i, \sigma}$ with $\sigma=1 . .2 N$ and we define an $S p(N)$-invariant bond operator:

$$
A_{i j}=\sum_{\sigma, \sigma^{\prime}=1 . .2 N} \mathcal{J}_{\sigma, \sigma^{\prime}} b_{i}^{\sigma} b_{j}^{\sigma^{\prime}}
$$

where the $2 N \times 2 N$ antisymmetric tensor $\mathcal{J}$ is block diagonal

$$
\mathcal{J}=\left[\begin{array}{cccccc}
0 & 1 & & & & \\
-1 & 0 & & & & \\
& & \ddots & & & \\
& & & 0 & 1 \\
& & & -1 & 0
\end{array}\right]
$$

and generalizes the $S U(2)$ antisymmetric tensor $\epsilon_{i j}(S U(2)$ is identical to $S p(1))$. Up to some constant the $S p(N)$ Hamiltonian is

$$
\mathcal{H}=-\frac{1}{N} \sum_{i j} J_{i j} A_{i j}^{\dagger} A_{i j}
$$

with the constraints

$$
\forall i \sum_{\sigma=1}^{2 N} b_{i \sigma}^{\dagger} b_{i \sigma}=n_{c}
$$

$n_{c}=2 S$ in the $S U(2)$ case and $n_{c} / N=\kappa$ will be kept constant when taking the large- $N$ limit. The partition function can be represented by an imaginary time functional integral:

$$
\begin{gathered}
Z=\int \mathcal{D}\left[\lambda_{i}, b_{i}^{\sigma}, b_{i}^{\dagger \sigma}\right] \exp \left(-\int_{0}^{\beta}\left(L_{0}+\mathcal{H}\right) d \tau\right) \\
L_{0}=\sum_{i \sigma} b_{i}^{\dagger \sigma}\left(\partial_{\tau}+i \lambda_{i}\right) b_{i \sigma}-i N \kappa \sum_{i} \lambda_{i}
\end{gathered}
$$


The $\lambda_{i}(\tau)$ are Lagrange multipliers that enforce the constraint Eq. 15 at every site. Bond degrees of freedom $Q_{i j}$ are introduced in order to decouple the bosons (Hubbard-Stratonovitch). The partition function is now

$$
Z=\int \mathcal{D}\left[Q_{i j}, \bar{Q}_{i j}, \lambda_{i}, b_{i}^{\sigma}, b_{i}^{\dagger \sigma}\right] \exp \left(-\int_{0}^{\beta}\left(L_{0}+L_{1}\right) d \tau\right)
$$

with

$$
L_{1}=\sum_{i j}\left[N \frac{\left|Q_{i j}\right|^{2}}{J_{i j}}-\left(A_{i j}^{\dagger} Q_{i j}+\text { h.c }\right)\right]
$$

so that a Gaussian integration of the $Q_{i j}$ gives back Eq. 16. The bond variable are $S p(N)$ invariant and they can take non-zero expectation values at a mean-field level without breaking the $S p(N)$ symmetry. As we explain below, they are however not gauge-invariant.

\subsubsection{Gauge invariance}

An important property of Eq. 18 is the $U(1)$ gauge invariance associated to the following transformations:

$$
\begin{aligned}
b_{i \sigma} & \rightarrow b_{i \sigma} e^{i \phi_{i}} \\
Q_{i j} & \rightarrow Q_{i j} e^{i\left(\phi_{i}+\phi_{j}\right)} \\
\lambda_{i} & \rightarrow \lambda_{i}-\partial_{\tau} \phi_{i}
\end{aligned}
$$

where $\phi_{i}(\tau)$ are arbitrary site- and imaginary-time-dependent angles. This gauge invariance comes from the the conservation of the local boson number and reflects the fact the magnitude of the spin is constant at each site. If we focus on the phase degrees of freedom of the bond variables, the Eq. 18 describes a system of charge-1 bosons coupled to a $U(1)$ lattice gauge theory $125 \mid 126$ These gauge degrees of freedom play a crucial role in the analysis of the fluctuations about mean-field solutions.

Effective action for the bond variables - The boson degrees of freedom can be integrated out to give an effective action for the bond variables:

$$
\begin{gathered}
Z=\int \mathcal{D}\left[Q_{i j}, \bar{Q}_{i j}, \lambda_{i}\right] \exp \left(-S^{\mathrm{eff}}\right) \\
S^{\mathrm{eff}}=N \int_{0}^{\beta}\left[\sum_{i j} \frac{\left|Q_{i j}\right|^{2}}{J_{i j}}-i \kappa \sum_{i} \lambda_{i}\right]-N \operatorname{Tr} \log G
\end{gathered}
$$


where $G^{-1}$ is the quadratic form which couples the bosons in Eq. 18 (propagator). It depends on the bond variables and on $\lambda_{i}$. We may write formally $G^{-1}=\partial_{\tau}-i \lambda-Q$. The term $N \operatorname{Tr} \log G$ is the free energy of the bosons in presence of the bond fields. By construction the action $S^{\mathrm{eff}}$ is gaugeinvariant with respect to the transformations of Eqs. 20 22. So far this is an exact formulation of the original model for arbitrary $N$.

\subsubsection{Mean-field $(N=\infty$ limit $)$}

Since $N$ factorizes (no flavor index is left in Eq. 24, $Z$ is dominated by the saddle point of $S^{\text {eff }}$ when $N$ is large. For simple models such as the first-neighbor antiferromagnet on the cubic lattice (any space dimension), the saddle point can be determined analytically. The $N=\infty$ limit is almost equivalent to the Schwinger-boson mean-field theory 1227128 This can otherwise be done numerically ${ }^{b}$ In this large- $N$ limit, two kinds of meanfield solutions can appear. For large enough $\kappa$ the bosons condense at some wave vector, the spectrum of the mean-field Hamiltonian is gapless. This corresponds physically to Néel long-range order. On the other hand, for smaller $\kappa$ (smaller "spin") the mean field Hamiltonian is gapped and the ground state preserves the $S p(N)$ symmetry. Fluctuations around the saddle point are not expected to change drastically the Néel ordered phases but they play an important role in the physics of $S p(N)$ symmetric phases. The following is a brief discussion of the effects of fluctuations in these non-magnetic phases.

\subsubsection{Fluctuations about the mean-field solution}

At the mean-field level described above some $Q_{i j}$ acquire a (static in all known cases) non-zero expectation value: $\left\langle Q_{i j}\right\rangle=\bar{Q}_{i j}$. For this reason such a state spontaneously breaks the local gauge invariance of Eqs. 20|21] and 22 . However this does not mean that the gauge degrees are all gapped and do not play any role at low energy. In fact, as remarked by Read and

\footnotetext{
b To our knowledge, all the saddle points considered so far are static (expectation values of the $Q_{i j}$ are time-independent) and the corresponding $\left\langle Q_{i j}\right\rangle$ could all be made real with an appropriate gauge transformation. There is no chiral order and the time-reversal symmetry is unbroken. The (oriented) sum of the complex phases of the bond variables around a plaquette defines a $U(1)$ flux. This flux is related to the solid angle formed by the spins and it vanishes in collinear as well as in coplanar states. In such cases the phases can be therefore be gauged away and the $\left\langle Q_{i j}\right\rangle$ can be made real. For this reason complex bond variables are usually not observed 129
} 
Sachdev, depending on the geometry of the lattice defined by the bonds where $Q_{i j} \neq 0$, some long-wavelength gapless gauge excitations survive and the associated fluctuations must be taken into account. More precisely, the fluctuations of the bond variables about the saddle point are decomposed into an amplitude and a phase

$$
Q_{i j}=\left(\bar{Q}_{i j}+q_{i j}\right) \exp \left(i \theta_{i j}\right)
$$

and we expand $S_{\text {eff }}$ with these new variables. Two cases must then be considered:

i) The lattice made of the sites connected by non-zero $\bar{Q}_{i j}$ bonds is bipartite. This is automatically the case if the original lattice defined by bonds where the exchange $J_{i j} \neq 0$ is bipartite. This can also be true if the original lattice is a non-bipartite lattice but some bonds have $\bar{Q}_{i j}=0$ so that the remaining lattice is bipartite. This is the case, for instance, in the $J_{1}-J_{2}$ model on the square lattice, $\frac{120}{1 n}$ some regions of the phase diagram of the Shastry-Sutherland mode $\sqrt{89}$ and in on the checkerboard Heisenberg model 104 Such configurations of the bond variables give collinear spin structures: spin-spin correlations can either be long-ranged (large $\kappa$, Néel phase) or short-ranged but in both cases the magnetic structure factor is peaked at a simple wave vector $\mathbf{k}_{0}$ such that $2 \mathbf{k}_{0}$ is a reciprocal lattice vector $\left(k_{0}=(\pi, 0), k_{0}=(0, \pi)\right.$ or $k_{0}=(\pi, \pi)$ in square geometries).

ii) The lattice made of the sites connected by non-zero $\bar{Q}_{i j}$ bonds is not bipartite. This happens in some phases of the $J_{1}-J_{2}-J_{3}$ model on the square lattice, $\frac{120}{20}$ on the triangular or kagome lattices ${ }^{130}$, in the Shastry-Sutherland model for some values of the exchange parameters 89 and on an anisotropic triangular lattice.131 Such mean-field states generically have planar but non-collinear spinspin correlations.

It is simple to check that case i) preserve a global continuous symmetry while such a symmetry is absent in ii). Consider the following global gauge transformation in case i) :

$$
\begin{gathered}
b_{i \sigma} \rightarrow b_{i \sigma} e^{i \phi} \quad b_{j \sigma} \rightarrow b_{j \sigma} e^{-i \phi} \\
Q_{i i^{\prime}} \rightarrow Q_{i i^{\prime}} e^{2 i \phi} \quad Q_{j j^{\prime}} \rightarrow Q_{j j^{\prime}} e^{-2 i \phi} \\
Q_{i j} \rightarrow Q_{i j}
\end{gathered}
$$


where $i, i^{\prime}$ belongs to sublattice $A$ and $j, j^{\prime}$ belongs to sublattice $B$. This transformation does not change the mean-field parameters $\bar{Q}_{i j}$. The only fields affected by this transformation are those connecting two sites on the same sublattice ${ }^{\mathrm{C}}$ They have a zero expectation values in case i) (or do not even exist if the physical lattice is itself bipartite). For this reason it is possible to make low-energy (and long-wavelength) gapless gauge excitations about the saddle-point by replacing the global staggered phase shift $\phi$ of Eq. 26 by a slowly varying (staggered) $\theta_{i j}$ (Eq. 25). A gradient expansion of the effective action performed at the appropriate points in the Brillouin zone for the phase fluctuations only involves gradients of $\theta$. The corresponding action is that of $U(1)$ lattice gauge theory coupled to charge- 1 boson (spinons).

\subsubsection{Topological effects - instantons and spontaneous dimerization}

So far only small fluctuations around the saddle point were considered and the contribution of topologically non-trivial gauge-field configurations were ignored. Consequently, the magnitude of the "spin" $n_{c} / 2$ was a continuous parameter and the information about the integer or half-integer (for instance) character of $S$ as disappeared. From Haldane's work on quantum spin chains and non-linear sigma model ${ }^{132}$ it is well known that Berry phases in spin systems give rise to topological terms in the low-energy effective action which can play a crucial role depending on the parity of $2 S$.

In non-linear sigma models in $2+1$ dimensions the Berry phase vanishes for configurations which are smooth on the scale of the lattice spacing $133[134$ (unlike the $1+1$ dimensional case). However "hedgehog" space-time singularities $\frac{133}{13}$ give non-trivial Berry phases. Read and Sachdev found that the closely related instantons of the effective $U(1)$ gauge theory described befor d also play a crucial role in the physics of the $S p(N)$ (as well as $S U(N))$ spin models.

The Berry phase associated to such a singular configuration depends on details of the lattice geometry. In the short-range ordered $(\pi, \pi)$ phase of the square lattice antiferromagnet this Berry phase is a multiple of $i n_{c} \pi / 2$.

\footnotetext{
${ }^{\mathrm{c}}$ In a gauge theory language the fields $Q_{i i^{\prime}}\left(\right.$ resp. $\left.Q_{j j^{\prime}}\right)$ of Eq. 27 transform like a charge-2 scalar for the $U(1)$ gauge field. Instead, from Eq. 26 the bosons (spinons) carry a charge 1 .

${ }^{\mathrm{d}}$ In that gauge theory associated to the phases of the link variables an instanton corresponds to a tunneling event during which the total magnetic field piercing the lattice is changed by $\pm 2 \pi$.
} 
Although dealing with a gas of interacting $(1 / r$ Coulomb-like potential) instantons is a difficult problem (see Ref. 119 and references therein), we can guess that the physics will depend on $n_{c} \bmod 4$. A detailed analysis 119 shows that when $n_{c} \neq 0$ mod 4 the instantons condense and spontaneously break the lattice translation symmetry. This generates a static electric field for the $U(1)$ gauge field. Since the electric field is coupled to the difference of amplitudes of the bond variables, such state acquires spatially inhomogeneous expectation values of the bond variables, it is a VBC and spinons are confined in pairs. In the $J_{1}-J_{2}$ model around $J_{2} / J_{1} \simeq 0.5$ the mean-field state is short-range ordered with correlations peaked at $(\pi, \pi)$. A columnar dimerized state is predicted by Read and Sachdev from this analysis of the fluctuations, in agreement with a number of numerical works on the $S U(2)$ $J_{1}-J_{2}$ spin- $\frac{1}{2}$ model.

In a recent work by Harada, Kawashima and Troyer $\frac{135}{13}$ the phase diagram of the (first neighbor - unfrustrated) $S U(N)$ antiferromagnet on the square lattice with $n_{c}=1$ was found to be in complete agreement with Read and Sachdev's predictions. They showed by quantum Monte Carlo simulations that for $N \leq 4$ the systems is Néel ordered whereas it is a columnar VBC for $N>5$. This provides an additional support to the field theory arguments described above. It also underlines that the mechanism of spontaneous symmetry breaking and formation of a VBC may come from quantum fluctuations only and that frustration is not always required (although it clearly enhances quantum fluctuations).

On the other hand when $n_{c}=0$ mod 4 the analysis of Read and Sachdev shows that fluctuations should not bring any broken lattice symmetry. Spinons are also confined and this state closely resembles the valence-bond solid (VBS) proposed by Affleck et al $\underline{61}$ as a possible ground state when the spin $S$ matches the coordination number $z$ according to $2 S=0 \bmod z$ (see 3.3 ).

\subsubsection{Deconfined phases}

Now we suppose that, starting from a mean-field solution with collinear correlations (case i), a parameter of the original spin model is varied so that the mean field solution is changed and some bonds $Q_{i i^{\prime}}\left(i\right.$ and $i^{\prime}$ belong to the same sublattice) acquire a non zero expectation value (case ii). In the framework of square lattice antiferromagnets, a finite third-neighbor coupling $\left(J_{3}\right)$ would be needed ${ }^{120}$ From the point of view of the longwavelength gauge fluctuations (related to the continuum limit of the phases 
$\left.\theta_{i j}\right)$ discussed above, the appearance of $\bar{Q}_{i i^{\prime}} \neq 0$ is equivalent to the condensation of a (Higgs) boson of charge 2. This is a spontaneous break down of the global $U(1)$ staggered symmetry of Eqs. 2628 down to a $\mathbb{Z}_{2}$ one since the field $Q_{i i}$ is not invariant under Eq. 27 except if $\phi=0$ or $\pi$. Based on results of Fradkin and Shenker ${ }^{[136}$ concerning confinement in compact lattice gauge theories coupled to matter, Read and Sachdev argued that this Higgs mechanism suppresses the low-energy gauge fluctuations and liberate the spinons. This confinement transition is described by a $\mathbb{Z}_{2}$ gauge theory. The suppression of the $U(1)$ gauge fluctuations also forbids the condensation of instantons discussed above and the ground state remains unique ${ }^{\text {e }}$ and bond variables have uniform expectation values. It is a genuine SL without any broken symmetry and deconfined spinons.

\section{Quantum Dimer Models}

In a previous section $\left(\$ 3\right.$ we showed that pairing spins- $\frac{1}{2}$ into singlets at short distances is a rather natural way to overcome frustration in Heisenberg antiferromagnets. QDM are defined in the Hilbert space of nearestneighbor valence-bond (or dimer) coverings of the lattice. By construction these models focus on the dynamics in the singlet space and ignore magnetic (gapped magnons or gapped spinons) excitations. For this reason they are (a priori) not appropriate to describe the physics of spin systems where magnetic excitations are gapless.

The Hamiltonian of a QDM usually contains kinetic as well as potential energy terms for these dimers. Such Hamiltonians can often be simpler than their spin parents and are amenable to several analytic treatments because of their close relations to classical dimer problems, $137|138| 139$ Ising models and $\mathbb{Z}_{2}$ gauge theory ${ }^{140|141| 142}$ These models can offer simple descriptions of VBC ${ }^{143}$ as well as RVB liquids $144 \mid 141$ It is in particular possible to write down some QDM that have a simple and exact VBC ground state with spontaneous broken symmetries (such as Rokhsar and Kivelson's model on the square lattice ${ }^{[143}$ with attractive potential energy only - in which case the exact ground state is very simple). Simple solvable QDM which have a dimer-liquid ground state can also be constructed $1 \frac{141}{}$

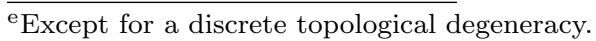




\subsection{Hamiltonian}

The first QDM was introduced by Rokhsar and Kivelson! ${ }^{143}$ It is defined by an Hamiltonian acting in the Hilbert space of first-neighbor dimer (valencebonds) coverings of the square lattice and reads:

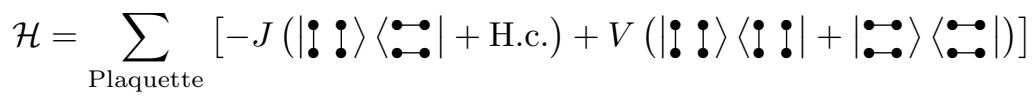

Flipping two parallel dimers around a square plaquette is the simplest dimer move on the square lattice and the $J$ terms precisely represent such dynamics. The $V$ terms are diagonal in the dimer basis and account for an attraction or repulsion between nearest-neighbor dimers. These are the two most local terms (respecting all lattice symmetries) which can be considered回

\subsection{Relation with spin- $\frac{1}{2}$ models}

There exists different interesting mappings between frustrated Ising models and QDM $\sqrt{145 \mid 142}$ Here, however, we focus on the relations between QDM and $S U(N)$ (or $S p(N)$ ) spin models in which dimers are related to singlet valence-bonds.

Overlap expansion. - A valence-bond state (product of two-spin singlets - belongs to the spin- $\frac{1}{2}$ Hilbert space) can be associated to any dimer covering $b$ Two such valence-bond states $|a\rangle$ and $|b\rangle$ are not orthogonal but, as first discussed by Sutherland $\frac{146}{14}$ their overlap decays exponentially with the length $L$ of the loops of their transition graphs (defined in \$5.3.1) as $|\langle a \mid b\rangle|=2^{1-L / 2}$. Rokhsar and Kivelson ${ }^{143}$ introduced a formal expansion parameter $x$ and replaced $|\langle a \mid b\rangle|$ by $2 x^{L}$. Their idea is that although $x=\frac{1}{\sqrt{2}}$ for physical $S U(2)$ spins, the physics of some models may be captured by the first orders of a small $x$ expansion. Truncating this expansion to order $x^{n}$ gives an effective Hamiltonian which contains local terms involving at most $n$ dimers ${ }^{c}$ In this approach the dimer states of the QDM

aThey were originally derived $\sqrt{143}$ as the lowest order terms of a formal overlap expansion (see 5.2 below) of the Heisenberg. In that calculation $J \sim x^{4}$ and $V \sim x^{8}$. Notice that a three-dimer kinetic term (extending over two neighboring plaquettes) is generated at order $x^{6}$ and is not included in Eq. 29

${ }^{\mathrm{b}}$ There is however a sign ambiguity (a valence-bond is antisymmetric under the exchange of both spins) that can be fixed by choosing an orientation on every bond.

${ }^{\mathrm{c}}$ The signs of the non-diagonal (kinetic) terms of the effective QDM obtained by such small $x$ expansion depends on the sign convention which was chosen to map valence- 
are in one-to-one correspondence with orthogonalized valence-bonds in the spin Hilbert space d

Fluctuations about large- $N$ saddle points. - From the argument above it could seem that the connection between spin- $\frac{1}{2}$ models and QDM relies on a variational approximation: the spin Hilbert space is restricted to the nearest-neighbor valence-bond subspace. This connection is in fact probably deeper, as some theories describing fluctuations about some large- $N$ saddle points ( $1 / N$ corrections) are equivalent to (generalized) QDM. This mapping was discussed by Read and Sachdev $\sqrt{123}$ for representation with $m=1$ (number of rows in the Young tableau of the $S U(N)$ representation), $N \rightarrow \infty$ and $n_{c} \sim \mathcal{O}(1)$, it leads to a generalized QDM where $n_{c}$ dimers emanate from each site. A QDM also describes $1 / N$ corrections in the case of the fermionid $S U(N)$ generalization ${ }^{116[124}$ of the Heisenberg model:

$$
\begin{aligned}
\mathcal{H} & =\frac{1}{N} \sum_{i j} J_{i j}: B_{i j}^{\dagger} B_{i j}: \\
& =-\frac{1}{N} \sum_{i j} J_{i j} B_{i j}^{\dagger} B_{i j}+\mathrm{cst} \\
\text { where } B_{i j} & =\sum_{\sigma=1}^{N} c_{j \sigma}^{\dagger} c_{i \sigma}
\end{aligned}
$$

and where the $c_{i \sigma}$ are $N$ flavors of fermions satisfying a constraint similar to Eq. 15 :

$$
\sum_{\sigma=1}^{N} c_{i \sigma}^{\dagger} c_{i \sigma}=N / 2
$$

Rokhsar showed 124 that in the $N \rightarrow \infty$ limit "dimerized states" (or Peierls states) becomes exact ground states of Eq. 30 for a large class of models ${ }^{f}$

bonds to dimers. An important question is to know whether, at least at the lowest non-trivial order, a sign convention giving the same sign for all kinetic terms exists (as in Eq. 29. This is the case on the square $\frac{143}{143}$ and triangular lattices 144

${ }^{\mathrm{d}}$ This implicitly assumes that the valence-bond states are linearly independent. This can be demonstrated on the square lattice and appears to be the case on the triangular and kagome lattices for large enough sizes. The operator which orthogonalizes the valencebond basis into the dimer basis is $\Omega^{-1 / 2}$ where $\Omega_{a, b}=\langle a \mid b\rangle$ is the overlap matrix.

eYoung tableau with $n_{c}=1$ column and $m=N / 2$ rows.

${ }^{\text {f }}$ Let $J_{0}$ be the largest value of the $J_{i j}$. Each dimerization (no site is left empty) where only bonds where $J_{i j}=J_{0}$ are occupied is a ground state. Here a "dimer" between to neighbors $i$ and $j$ consists of a $S U(N)$ singlet made with $N$ fermions (one of each flavor) 
Quite naturally, $1 / N$ corrections will induce a dynamics into this subspace of dimerized states; it can be described by a QDM (with kinetic energy terms only at this order). At lowest order, on the square lattice, a kinetic term identical to the $J$ term in Eq. 29 is generated and naturally favors a columnar or resonating-plaquette crystal (in agreement with a number of works on the spin- $\frac{1}{2}$ model). The same arguments were discussed for the kagome lattice ${ }^{147}$ In that case the leading $1 / N$ corrections to the fermionic saddle point generate three-dimer moves around hexagons and stabilize a crystal of resonating hexagons 8 This formalism was also applied to the checkerboard mode ${ }^{\sqrt{98}}$ to conclude to the presence of a VBC phase.

\subsection{Square lattice}

The phase diagram of the Rokhsar and Kivelson's square lattice QDM is shown Fig. 3. Since a change in the signs of the basis dimer configurations can change $J$ into $-J$ (see Ref. ${ }^{143}$ ) we will choose $J>0$ without loss of generality.

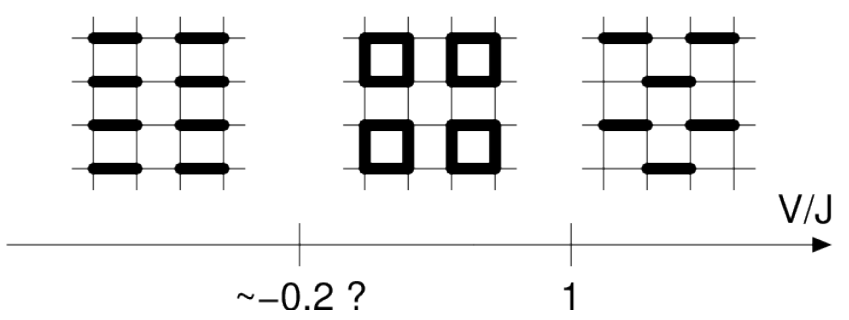

Fig. 3. Schematic phase diagram of the square lattice QDM. The possible location of an intermediate plaquette phase is taken from the work of Leung et al! $[148$

\subsubsection{Transition graphs and topological sectors}

In order to understand the particularities of QDM on bipartite lattices it is useful to describe how the set of dimer coverings splits into topological

hoping back and forth between $i$ and $j$. It is constructed from $\prod_{\sigma=1}^{N}\left(c_{i \sigma}^{\dagger}+c_{j \sigma}^{\dagger}\right)|0\rangle$. by projecting out the components which do not satisfy Eq. 32 . Notice however that in the $N \rightarrow \infty$ limit the relative fluctuations of the total number of fermion on each site are of order $1 / \sqrt{N}$ and can be neglected.

g To our knowledge however there is no clear evidence of such ordering in the spin- $\frac{1}{2}$ case. 
sectors. To do so we first have to define transition graphs: the transition graph of two dimer coverings $c$ and $c^{\prime}$ is obtained by superimposing $c$ and $c^{\prime}$ on the top of each other; it defines a set of non-intersecting loops covering the lattice. On each bond where the dimers of $c$ and $c^{\prime}$ match, a trivial loop of length 2 is obtained. When the lattice is bipartite (two sublattices $A$ and $B$ ) these loops can be oriented in the following way: any dimer belonging to $c$ is oriented from $A$ to $B$ and dimers of $c^{\prime}$ are oriented $B \rightarrow$ $A$. The transition graph is then made of loops of the type $A \rightarrow B \rightarrow$ $A \rightarrow B \cdots$. With periodic boundary conditions two winding numbers 143 $-L / 2 \leq \Omega_{x}, \Omega_{y} \leq L / 2$ are associated to such a transition graph $(L \times L$ sites). $\Omega_{x}$ (resp. $\Omega_{y}$ ) is the net number of topologically non-trivial loops (clockwise minus counterclockwise) encircling the torus in the $x$ (resp. $y$ ) direction.

Dimer coverings can be grouped into different topological sectors. By definition two dimer coverings belong to the same sector if they can be transformed into each other by repeated actions of local dimer moves (the transition graph associated to each movement does not wind around the whole system if it has periodic boundary conditions). On the square lattice two-dimer moves are sufficient to connect any two states in the same sector; that is the Hamiltonian Eq. 29 is ergodic within each topological sector. In a torus geometry, $c$ and $c^{\prime}$ belongs to the same sector if and only if their transition graph has winding numbers $\Omega_{x}=\Omega_{y}=0$. The different topological sectors can be labeled by their winding numbers with respect to some reference columnar configuration. Their number is of order $\mathcal{O}\left(L^{2}\right)$ for a system of linear size $L$.

\subsubsection{Staggered $V B C$ for $V / J>1$}

When $V$ is sufficiently large the system tries to minimize the number of parallel dimers. The staggered configuration shown Fig. 3 has no such flippable plaquette. It is always a zero-energy eigenstate of Eq. 29 and becomes a ground state for $J \geq V$. It breaks several lattice symmetries (four-fold degenerate) and is a VBC.

The expectation value of the energy per plaquette satisfies $\min (0, V-$ $J) \leq E_{0} / N_{p} \leq \max (0, V+J)$. For $V / J>1$ this gives $0 \leq E_{0} / N_{p}$ and any zero-energy state saturates this lower bound and is a therefore a ground state. One should however notice that it is possible to make zero-energy domain walls in this VBC since the state shown Fig. 4 is also annihilated by the Hamiltonian. No local dimer movement can take place in the staggered 
VBC (with or without domain walls). Each of these states form a topological sector with a single configuration which has $\left|\Omega_{x}\right|+\left|\Omega_{y}\right|=L / 2$ with respect to a columnar state.

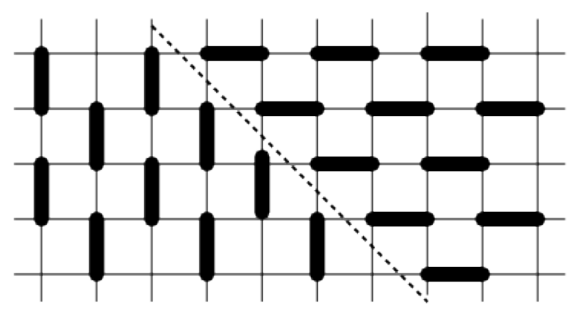

Fig. 4. Domain wall in a staggered VBC.

\subsubsection{Columnar crystal for $V<0$}

When parallel dimers attract each other the system tries to maximize the number of flippable plaquettes. Columnar configurations as shown on the left side of Fig. 3 do maximize this number. Such a VBC is exactly realized for $V<0$ and $J=0$. Elementary excitations consist of a pair of (say) vertical dimers in a background of vertical columns of horizontal dimers. Such excitations are gapped $(\Delta E=2|V|)$ and this VBC phase will survive to the inclusion of a finite $J$ term. Notice that unlike the staggered VBC presented in the previous paragraph the columnar dimer configuration is not an exact eigenstate when $J \neq 0$. The exact diagonalizations data of Leung et al148 suggest that the columnar phase may disappear around a critical value $V / J \simeq-0.2$.

\subsubsection{Plaquette phase}

When the kinetic energy dominates $(|V| \ll J)$ the system will try to maximize the number of resonating plaquettes |||\rangle$+|=\rangle$. This can be achieved through the resonating plaquette crystal shown Fig. 3 and the numerical work (exact diagonalizations up to $8 \times 8$ sites) of Leung et a that this phase is realized in an interval $-0.2 \leq V / J \leq 1$. Although this model would not suffer from the fermion sign problem we are not aware of any QMC simulation on this model. 


\subsubsection{Rokhsar-Kivelson point}

The point $J=V$ (Rokhsar-Kivelson (RK) point) plays a special role. As remarked by Rokhsar and Kivelson $\frac{143}{}$ the equal-amplitude superposition of all dimerizations in a given topological sector is an exact ground state. When $J=V$ the Hamiltonian can be written as a sum of projectors:

$$
\begin{aligned}
\mathcal{H}_{J=V=1} & =\sum_{p}\left|\Psi_{p}\right\rangle\left\langle\Psi_{p}\right| \\
\left|\Psi_{p}\right\rangle & =|\mathfrak{\bullet}:\rangle-|\bullet \bullet\rangle
\end{aligned}
$$

The linear superposition of all dimer coverings belonging to a given sector $\Omega$

$$
|0\rangle=\sum_{c \in \Omega}|c\rangle
$$

is annihilated by Eq. 33 and is therefore a ground state. The argument is the following. Consider a plaquette $p$ and a configuration $|c\rangle$. If $|c\rangle$ has one or no dimer at all on the edges of $p$ we have $\left\langle\Psi_{p} \mid c\right\rangle=0$. If two dimers are present, then there exists a configuration $\left|c^{\prime}\right\rangle$ in the same sector which only differ from $|c\rangle$ by a two-dimer flip on $p$. In such a case the combination $|c\rangle+\left|c^{\prime}\right\rangle$ is again orthogonal to $\left|\Psi_{p}\right\rangle$. This shows that $H|0\rangle=0$.

When open boundary conditions are considered (this restricts the topological sector to $\left.\Omega_{x}=\Omega_{y}=0\right)$ the RK state is the linear combination of an exponential number of configurations $\mathrm{h}^{\mathrm{h}}$ This is very different from the crystalline states considered so far where some periodic configurations were favored and it closely resembles Anderson's RVB picture. As we shall see this RK state is not a "true" liquid on the square lattice since dimerdimer correlations are not short-range but algebraically decaying $\left(\sim 1 / r^{2}\right)$ with distance. The calculation of dimer-dimer correlations in the RK state (Eq. 35) maps onto a classical dimer problem solved by Kasteleyn $137 / 139$ Fisher ${ }^{138}$ and Fisher and Stephenson. ${ }^{[149}$ From this Rokhsar and Kivelson ${ }^{143}$ constructed gapless excitations (in single-mode approximation) which dispersion relation vanishes as $\mathbf{k}^{2}$ at small momentum (the origin is taken at $(\pi, \pi)$ ). Quoting Rokhsar and Kivelson, 143 these excitations (dubbed "resonons") are the Goldstone mode of the gauge symmetry which allows the phases of the different topological sectors to be varied without changing the energy. Another mode of gapless excitations (around $(\pi, 0)$ and $(0, \pi)$

$\mathrm{h}_{\mathrm{This}}$ is also true for periodic boundary conditions provided the two winding numbers do not scale like the linear size $L$. 
in the Brillouin zone), specific to the fact that the ground state has critical (algebraic) dimer-dimer correlations, was recently discovered 150

The QDM on the square lattice is thus believed to be ordered (VBC) everywhere except at the RK point $(J=V)$ where it has quasi long-range (critical) dimer-dimer correlations.

\subsection{Hexagonal lattice}

The QDM on the honeycomb lattice was studied by Moessner, Sondhi and Chandra, $\frac{151}{1 n}$ in particular with Monte Carlo simulations. The phase diagram is very similar to the square lattice-case discussed above. It possesses three crystalline phases (Fig. 5) and has algebraically decaying dimer-dimer correlations at the Rokhsar Kivelson point (where the ground state in each sector has the same form as Eq. 35). The absence of liquid phase (with exponentially decaying $2 n$-mer- $2 n$-mer correlations) is believed to prevail in bipartite lattices. This relation between the absence of a deconfined dimer liquid phase $\mathrm{i}^{\mathrm{i}}$ and the bipartite character of the lattice as been discussed by several authors $\frac{152}{152}$ and is related to the possibility of a height representation $\frac{153}{15}$ of dimer coverings when the lattice is bipartite ${ }^{j}$

\footnotetext{
${ }^{\mathrm{i}}$ Bipartiteness seems to forbid deconfinement but not short-range dimer-dimer correlations. The 4-8 lattice (squares and octogons) is an example where dimer-dimer correlations are short-range. We thank R. Moessner for pointing this to us. On this lattice the equal-amplitude superposition of all coverings would be similar to an explicit VBC wave function (thus confining). Such situations are only possible when the number of sites is even in the unit cell.

${ }^{\mathrm{j}}$ Consider a bipartite lattice with coordination number $z$. For each dimer covering we can associate integers (heights) on the dual lattice by the following rule. Set the height to be zero on a plaquette at the origin. The height is then defined on the whole lattice by turning clockwise (resp. counterclockwise) around sites of the $A$-sublattice (resp. $B$ sublattice) and changing the height by $z-1$ when crossing a dimer and by -1 when crossing an empty bond. It is simple to check the difference of heights $\delta h(x)=h_{1}(x)-h_{2}(x)$ between two dimerizations is constant inside each loop of their transition graph. Notice that the loops of a transition graph can be naturally oriented on a bipartite lattice. Then, $\delta h(x)$ changes by $+z$ (resp. $-z$ ) when crossing a clockwise (resp. counterclockwise) loop of the transition graph. Columnar dimerizations have an averaged height which is flat and staggered ones have the maximum tilt. The winding numbers $\left(\Omega_{x}, \Omega_{y}\right)$ correspond to the average height difference between both sides of the sample. The kinetic energy terms of Eqs. 29 and 36 change the height of the corresponding plaquette by $\pm z$ and the potential terms $(V>0)$ favor tilted configurations.
} 


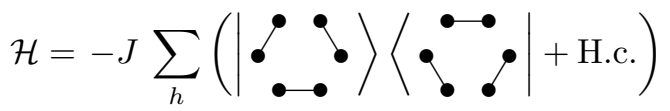

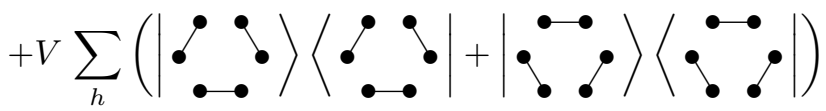

Fouet et al ${ }^{97}$ studied the spin- $\frac{1}{2} J_{1}-J_{2}-J_{3}$ model on the hexagonal lattice by exact diagonalizations and found evidences of a staggered VBC of the type predicted for $V / J>1$ by Moessner et al $\frac{151}{15}$ in the QDM. Other phases (Néel ordered phase and a possible short-range RVB SL) are also present in the spin- $\frac{1}{2}$ model. ${ }^{97}$

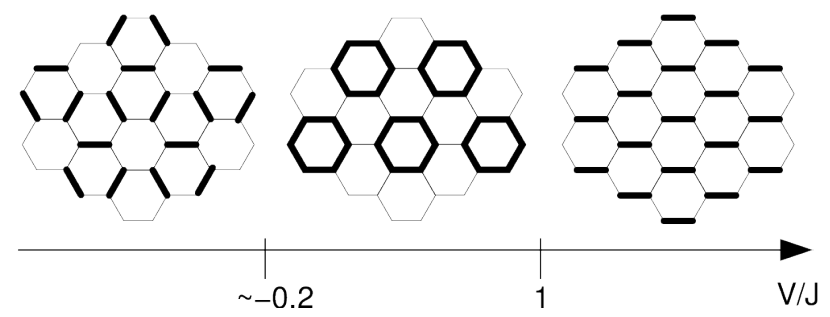

Fig. 5. Phase diagram of the hexagonal QDM obtained by Moessner et al $!^{151}$ Although the VBC shown for $V<0$ do not have all dimers parallel to the same direction it is equivalent to the columnar $\mathrm{VBC}$ found in the square lattice QDM because it maximizes the number of flippable plaquettes. It also corresponds to the ordering pattern predicted in the large- $N$ theory of Ref. 119 As for the $\mathrm{VBC}$ obtained for $V / J>1$, it is the hexagonal counterpart of the staggered $\mathrm{VBC}$ of the square lattice (no flippable plaquette, exact eigenstate and maximum tilt in a height representation).

\subsection{Triangular lattice}

The most local dimer Hamiltonian on the triangular lattice contains kinetic and potential two-dimer terms on each rhombus; it was studied by Moessner and Sondhi! 144

$$
\begin{aligned}
& \mathcal{H}=-J \sum_{r}(|\bullet \bullet\rangle\langle\bullet \bullet|+\text { H.c. }) \\
& +V \sum_{r}(|\bullet \bullet\rangle\langle\bullet \bullet|+| \bullet \bullet\rangle\langle\bullet|)
\end{aligned}
$$

where the sums run over all rhombi $r$ of the lattice (with three possible orientations). This model was shown to possess (at least) three crystalline 
phases, including a columnar and staggered one (Fig. 6) as in the two previous examples. An additional VBC (with resonating diamonds plaquettes) with a large unit cell (12 sites) was also predicted around $V=0$. When $V<0$ and $J=0$ the ground state is highly degenerate since it is possible, from an ordered columnar configuration, to shift all the dimers along any straight line without changing the number of flippable plaquettes (contrary to the square lattice case). However, an infinitesimal $J$ is expected to lift this degeneracy and to order the ground state in a columnar way.

The phenomenology of these ordered phases is that of usual VBC and we refer to the original paper ${ }^{144}$ for details.

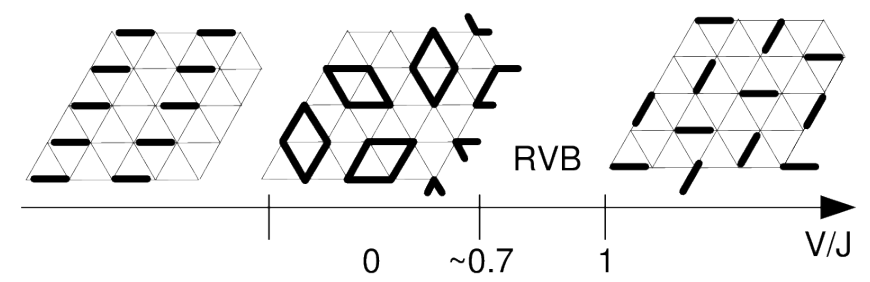

Fig. 6. Phase diagram of the triangular lattice QDM obtained by Moessner and Sondhi 144

\subsubsection{RVB liquid at the RK point}

The new physics of this model appears through the existence of a liquid phase in the interval $0.7 \lesssim V / J \leq 1$. As for the two previous QDM the ground states are exactly known at the RK point $J=V$. As before dimer-dimer correlations are obtained exactly at this point by a Pfaffian calculation $144|154| 155$ but the result shows a finite correlation length. From their Monte Carlo simulations Moessner et al! ${ }^{[44}$ argued that the spectrum is gapped at the RK point and that this gap persists down to $V / J \simeq 2 / 3$, that is over a finite range of coupling. This picture is consistent with the exact diagonalizations performed on this model $156 \mid 157$

\subsubsection{Topological order}

When the lattice is not bipartite the loops of a transition graph can no longer be oriented. The winding numbers $\Omega_{x}$ and $\Omega_{y}$ are now defined as the (positive) number of non-trivial loop around $x$ and $y$ in the transition graph 
with a reference configuration (say columnar). These two integers are not conserved by local dimer moves. They are however conserved modulo two, which leaves only four sectors ${ }^{\mathrm{k}}$ Consequently the dimer liquid ground state is four-fold degenerate at the RK point. This degeneracy holds exactly at the RK point even on finite-size samples but it is expected to hold in the thermodynamic limit in the whole liquid phase $(0.7 \lesssim V / J \leq 1)$.

Conventional orders are often associated to a spontaneously broken symmetry and lead to ground state degeneracies in the thermodynamic limit. The four-fold degeneracy discussed above is the signature of some kind of order, called topological order ${ }^{[158}$ The peculiarity of this order is that it is not associated to any local order parameter: a local observable cannot decide whether a given dimerization is in one sector or another. The existence of topological order is intimately associated to the fractionalized nature of the elementary excitations (see below). In the case of a RVB dimer liquid these excitations have been known to be Ising vortices for a long time $\underline{159 \mid 160}$ (dubbed visons in the recent literature $\underline{161 \mid 162}$ ). We will now discuss these excitations in more details in the framework of a QDM which realizes the same dimer liquid phase but for which not only the ground state but all the eigenstates are known exactly.

\subsection{Solvable $Q D M$ on the kagome lattice}

An exactly solvable QDM on the kagome lattice was introduced by D. Serban, V. Pasquier and one of us $\frac{141}{10}$ It offers a very simple and explicit realization of the ideas discussed above (visons, topological order etc.).

\subsubsection{Hamiltonian}

The kagome lattice QDM introduced in Ref. ${ }^{141}$ contains only kinetic terms and has no external parameter. The Hamiltonian reads:

$$
\begin{aligned}
\mathcal{H} & =-\sum_{h} \sigma^{x}(h) \\
\text { where } \sigma^{x}(h) & =\sum_{\alpha=1}^{32}\left|d_{\alpha}(h)\right\rangle\left\langle\bar{d}_{\alpha}(h)\right|+\text { H.c }
\end{aligned}
$$

\footnotetext{
${ }^{\mathrm{k}}$ The two-dimer moves included in Eq. 37 are not sufficient to guaranty ergodicity with each of the four sectors. Staggered states (12-fold degenerate - not $\mathcal{O}(L)$ like on the square lattice) have no flippable plaquette but can be connected to other states with four-dimer moves 144
} 
The sum runs over the 32 loops on the lattice which enclose a single hexagon and around which dimers can be moved (see Table 1 for the 8 inequivalent loops). The shortest loop is the hexagon itself, it involves 3 dimers. 4,5 and 6-dimers moves are also possible by including 2, 4 and 6 additional triangles (the loop length must be even). The largest loop is the star. For each loop $\alpha$ we associate the two ways dimers can be placed along that loop: $\left|d_{\alpha}(h)\right\rangle$ and $\left|\bar{d}_{\alpha}(h)\right\rangle$. Notice that $\sigma^{x}(h)$ measures the relative phases of dimer configurations displaying respectively the $d_{\alpha}(h)$ and $\bar{d}_{\alpha}(h)$ patterns in the wave function.

Table 1. The 8 different classes of loops which can surround an hexagon of the kagome lattice. Including all possible symmetries we find 32 possible loops. The first column indicates the number of dimers involved in the coherent motion around the hexagon.

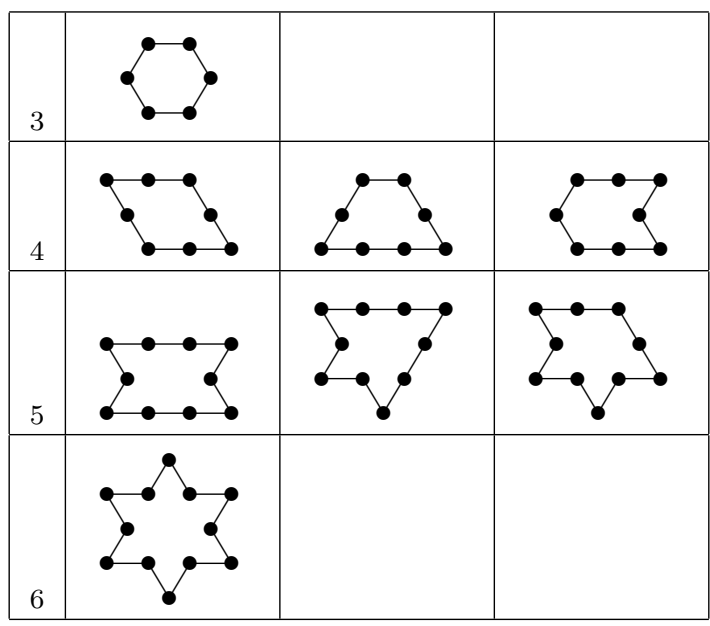

\subsubsection{RK ground state}

As for the QDM discussed previously the ground state of this Hamiltonian is the equal amplitude superposition of all dimer coverings belonging to a given topological sector (as on the triangular lattice there are four sectors). This can be readily shown by writing $\mathcal{H}$ as a sum of projectors:

$$
\mathcal{H}=-N_{h}+\sum_{h} \sum_{\alpha=1}^{32}\left[\left|d_{\alpha}(h)\right\rangle-\left|\bar{d}_{\alpha}(h)\right\rangle\right]\left[\left\langle d_{\alpha}(h)\right|-\left\langle\bar{d}_{\alpha}(h)\right|\right]
$$


where $N_{h}$ is the number of hexagons on the lattice. When expanding the products the diagonal terms give a simple constant since

$$
\sum_{\alpha=1}^{32}\left|d_{\alpha}\right\rangle\left\langle d_{\alpha}|+| \bar{d}_{\alpha}\right\rangle\left\langle\bar{d}_{\alpha}\right|=1
$$

This reflects the fact that, for any dimerization, the dimers on hexagon $h$ match one and only one of the $2 \times 32$ patterns $\left\{d_{\alpha}, \bar{d}_{\alpha}\right\}$.

Unlike the square or triangular case, the RK ground states $|0\rangle=$ $\sum_{c \in \Omega}|c\rangle$ are not degenerate with some staggered VBC This means that the Hamiltonian of Eq. 38 is not at a phase transition to a VBC. As we will explain it is inside a liquid RVB phase.

The RK wave function can be viewed as dimer condensate. It is similar to the ground state of liquid ${ }^{4} \mathrm{He}$ which has the same positive amplitude for any configuration and its permuted images $\frac{163}{163}$ An important difference, however, is that the QDM state is incompressible and cannot sustain acoustic phonons. This can be related to the fact that the $U(1)$ symmetry of the Bose liquid is absent in the QDM on non-bipartite lattices. It is replaced instead by a discrete $\mathbb{Z}_{2}$ gauge symmetry (see $\$ 5.6 .7$ below).

\subsubsection{Ising pseudo-spin variables}

The kinetic energy operators $\sigma^{x}$ defined in Eq. 39 commute with each other. This is obvious when two such operators act on remote hexagons but it also holds for neighboring ones. This property can easily be demonstrated with the help of the arrow representation of dimer coverings introduced by Zeng and Elser ${ }^{164}$ This mapping of kagome dimerizations to arrows on the bonds of the honeycomb lattice is illustrated Fig. 7 Each arrow has two possible directions: it points toward the interior of one of the two neighboring triangles. If site $i$ belongs to a dimer $(i, j)$ its arrow must point toward the triangle the site $j$ belongs to. A dimer covering can be constructed from any arrow configuration provided that the number of outgoing arrows is odd ( 1 or 3$)$ on every triangle.

The operators $\sigma^{x}$ have a particularly simple meaning in terms of the arrow degrees of freedom: $\sigma^{x}(h)$ flips the 6 arrows sitting on $h \mathrm{~m}$ It is then

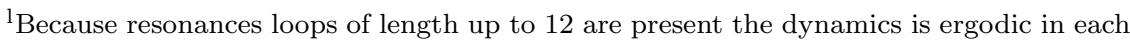
of the four topological sectors 141

${ }^{\mathrm{m}}$ Flipping all the arrows around any closed loop (such as around an hexagon) preserves the local constraint imposed on arrow configurations. Flipping the arrows around a topologically non-trivial loop changes the topological sector.
} 


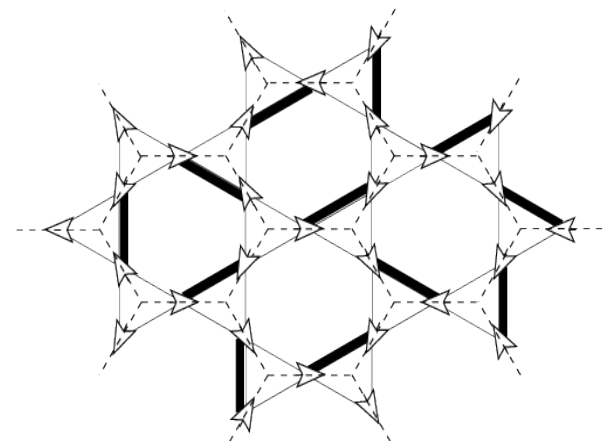

Fig. 7. A dimer covering on the kagome lattice and the corresponding arrows. Dashed lines: honeycomb lattice.

clear that the $\sigma^{x}$ commute and that $\sigma^{x}(h)^{2}=1$. In fact these operators can be used as Ising pseudo-spin variables and the Hamiltonian now describes non-interacting pseudo-spins in a uniform magnetic field pointing in the $x$ direction. In the ground state we have $\sigma^{x}(h)=1$ on every hexagon.

\subsubsection{Dimer-dimer correlations}

The ground state is the most possible disordered dimer liquid as the dimerdimer correlations strictly vanish beyond a few lattice spacings. Such correlations can be computed by the Pfaffian method. On the kagome lattice the determinant of the Kasteleyn matrix (which is directly related to the partition function of the classical dimers problem) is exactly constant in Fourier space $\frac{165}{16}$ Since dimer-dimer correlations are obtained from the Fourier transform of the inverse of this determinant, they turn out to be strictly zero beyond a few lattice spacings (as soon as the two bonds do not touch a common triangle) 141 This result can also be obtained by a simpler argument $141 \mid 166$ using the $\sigma^{x}$ operators. This result is related to the kagome geometry $\rrbracket^{n}$ This absence of long-range dimer-dimer correlations demonstrates that the RK state is a dimer liquid and that it breaks no lattice symmetry.

On the triangular lattice, even at high temperature, dimer-dimer correlations decay exponentially with distance but these correlations remain finite at any distance. On the square lattice such correlations are even larger

${ }^{\mathrm{n}}$ The model of Eq. 38 can be generalized to any lattice made of corner-sharing triangles!141 
because they decay only as a power law. This means that the infinite hardcore dimer repulsion makes QDM non-trivial even at infinite temperature; dimers cannot be free when they are fully-packed. From this point of view we see that the kagome lattice is particular: it is as close as possible to a free dimer gas, except for non-trivial correlations over a few lattice spacings. This is a reason why dimer coverings on the kagome lattice can be handled with independent pseudo-spin variables and why the RK state on this lattice is the most possible disordered RVB liquid.

\subsubsection{Visons excitations}

The $\sigma^{x}$ operators can be simultaneously diagonalized but they must satisfy the global constraint $\prod_{h} \sigma^{x}(h)=1$ since this product flips every arrow twice. It must therefore leave all dimerizations unchanged. The lowest excitations have therefore an energy 4 above the ground state and they are made of a pair of hexagons $a$ and $b$ in a $\sigma^{x}(a)=\sigma^{x}(b)=-1$ state. $a$ and $b$ are the locations of two Ising vortices (or visons $s^{161 \mid 162}$ ). As remarked before this means that the relative phases of the configurations with $d_{\alpha}(h)$ and $\bar{d}_{\alpha}(h)$ patterns have now changed sign. The corresponding wave function is obtained in the following way. Consider a string $\Omega$ which goes from $a$ to $b$ (see Fig. 8 ) and let $\Omega(a, b)$ be the operator which measures the parity \pm 1 of the number of dimers crossing that string. $\Omega(a, b)$ commutes with all $\sigma^{x}(h)$, except for the ends of the string: $\sigma^{x}(a) \Omega(a, b)=-\Omega(a, b) \sigma^{x}(a)$. A dimer move changes the sign of $\Omega(a, b)$ if and only if the associated loop crosses the string an odd number of times, which can only be done by surrounding one end of the string. This shows that $\Omega(a, b)$ flips the $\sigma^{x}$ in $a$ and $b 0^{\circ}$ As the RK ground state $|0\rangle, \Omega(a, b)|0\rangle$ is a linear combination of all dimer configurations belonging to one sector. However the amplitudes are now 1 and -1 depending on the number of dimers crossing $\Omega$. This wave function therefore has nodes, it is an excited state of energy 4 with two vortices in $a$ and $b$. It is easy to see that a different choice $\Omega^{\prime}$ for the string connecting $a$ and $b$ gives the same state up to a global sign which depends on the parity of the number of kagome sites enclosed by $\Omega \cup \Omega^{\prime}$.

These vortex excitations carry a $\mathbb{Z}_{2}$ charge since attempting to put two vortices on the same hexagon does not change the state. Such excitations are not local in terms of the dimer degrees of freedom. Indeed, determining the sign of a given dimerization in a state with two visons which are far

${ }^{\circ}$ Up to a global sign (reference dependent) $\Omega(a, b)$ is equal to $\sigma^{z}(a) \sigma^{z}(b)$ where the $\sigma^{z}$ operators are those introduced by Zeng and Elser. 
apart requires the knowledge of the dimer locations along the whole string connecting the two vortex cores. In this model the visons appear to be static and non-interacting. This is a particularity of this solvable model but the existence of gapped vison excitations is believed to be a robust property of RVB liquids. In more realistic models the visons will acquire a dynamics and a dispersion relation but will remain gapped P They will also have some interactions with each other but should remain deconfined. This property is particularly clear in the kagome QDM: visons are necessarily created by pairs but the energy is independent of their relative distances.

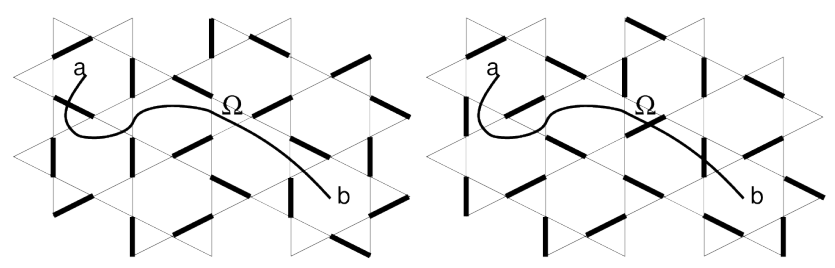

Fig. 8. A pair of visons (located in $a$ and $b$ ) is created by applying to the RK wave function a factor $(-1)$ for each dimer crossing the string $\Omega$. The dimerization shown there on the left appears in the linear superposition of the two-vison state with the sign -1 whereas the one on the right has the sign +1 .

The Ising vortices also offer a simple picture of the topological degeneracy. Consider a ground state $|+\rangle$ of the model which lives in the sector where the winding number $\Omega_{y}$ (with respect to some arbitrary but fixed dimerization) is even. Another ground state $|-\rangle$ is obtained in the odd- $\Omega_{y}$ sector. Now consider the combination $|0\rangle=|+\rangle+|-\rangle$ and apply the operator $\Omega\left(0, L_{x}\right)$ corresponding to a closed loop surrounding the torus in the $x$ direction. This amounts to creating a pair of nearby visons at the origin, taking one of them around the torus in the $x$ direction and annihilating them. This can also be viewed as the creation of a vison in one hole of the torus (with no energy cost). It is simple to check that $\Omega\left(0, L_{x}\right)|0\rangle=|+\rangle-|-\rangle$ (up to an irrelevant global sign). This provides a simple relation between the vison-pair creation operator and the existence of two topologically distinct ground states $|+\rangle+|-\rangle$ and $|+\rangle-|-\rangle$.

PIt is possible to add potential energy terms to Eq. 38 to drive the system outside of the liquid phase and this transition corresponds to a vison condensation. 


\subsubsection{Spinons deconfinement}

We assume that dimers represent "dressed" singlet valence-bonds, as in the overlap expansion $(\$ 5.2)$. Since the Hilbert space is made of fully-packed dimer coverings the model of Eq. 38 only describes spin-singlet states. However, as any QDM, it can be extended to include static holes or spinons. Configurations with unpaired sites (spinon or holon) are now allowed but the kinetic terms of the original Hamiltonian which loop passes on an empty site gives zero. Consider a system with two static spinons in $x$ and $y$. As on the square $\frac{143}{1}$ and triangular lattices $\frac{144}{14}$ at the RK point the exact ground state $|x, y\rangle$ remains the sum of all dimer coverings and the ground state energy is independent of the distance between the two spinons (except at very short distance if they belong to a common hexagon). This is a first indication that RVB spin liquid has deconfined spin- $\frac{1}{2}$ excitations (spinons). In the QDM language these excitations are simply unpaired sites in a dimer liquid background. Such unpaired sites are necessarily created by pairs but they can then propagate freely (no attractive potential) when they are sufficiently far apart.

Another calculation allows to test the deconfinement properties of a dimer liquid. We consider the state $|\psi\rangle=\sum_{\mathbf{r} \neq 0}|0, \mathbf{r}\rangle$ where $|0, \mathbf{r}\rangle$ is the (un-normalized) ground state with two spinons in 0 and $\mathbf{r}$. The probability to find a spinon in $\mathbf{r}$ in the $|\psi\rangle$ can be obtained by the relatively involved calculation of the monomer correlation a with Pfaffians. One the square lattice this probability goes to zero as $1 / \sqrt{r}[149$ This shows that the second spinon is (quasi-) confined in the vicinity of the first one on the square lattice because escaping far away represents a large "entropy" cost in the dimer background. On the triangular lattice it goes exponentially to a constant 154 This result is a signature of deconfinement. In fact the same signature can be obtained on the kagome lattice without any technical calculation since the monomer correlation is exactly $1 / 4$ at any distance 167

If unpaired sites are allowed one can describe spinons or holons. Unfortunately in the presence of simple kinetic energy terms for these objects the model can no longer be solved. However one can consider a static spinon and its interaction with visons: when the spinon is adiabatically taken around a vison the dimers are shifted along a path encircling the vison. Because the vison wave function is particularly simple in this model it is easy to check that this multiplies the wave function by a factor -1 . This is the signature

${ }^{\mathrm{q}}$ Ratio of number of dimer coverings with two holes in 0 and $\mathbf{r}$ to the number without hole. 
of a long-range statistical interaction ${ }^{160 \mid 159}$ between visons and spinons (or holons). In more realistic models, as long as the visons are gapped excitations the spinons are expected to be deconfined. On the other hand if the visons condense their long-range statistical interaction with spinons frustrates their motion. This is no longer true if they propagate in pairs, in which case they are not sensitive any more to visons (see Ref. 141 for an extension of the present QDM with a vison condensation). This simple physical picture illustrates the relation between vison condensation and spinon confinement.

\subsection{7. $\mathbb{Z}_{2}$ gauge theory}

The forces responsible for confinement are usually associated to gauge fields and their fluctuations. Whereas $U(1)$ compact gauge theories are generically confining in $2+1$ dimensions, $168|136| \mathbb{Z}_{2}$ gauge theories are known to possess deconfined phases ${ }^{[169}$ For this reason some attention has been paid to the connections between $\mathbb{Z}_{2}$ theories and fractionalized phases in $2 \mathrm{D}$ electronic systems. $\frac{161}{1}$

It is known ${ }^{140}$ that QDM can be obtained as special limits of $\mathbb{Z}_{2}$ gauge theories, the gauge variable being the dimer number on a bond. However, on the kagome lattice this connection can be made exact and completely explicit since there is a one to one correspondence between dimer coverings and physical states (i.e. gauge-invariant) of a $\mathbb{Z}_{2}$ gauge theory ${ }^{[141]} \mathrm{In}$ this mapping the gauge fields are Ising variables living on the link of the honeycomb lattice (i.e. kagome sites) and are constructed from the arrows described previously. As for the constraints of gauge invariance they correspond to the odd parity of the number of outgoing arrows on every triangles. The $\sigma^{x}$ operator used to define a solvable QDM translate into a gauge-invariant plaquette operator for the gauge degrees of freedom (product of the Ising gauge variables around an hexagon). With this mapping the visons appear to be vortices in the $\mathbb{Z}_{2}$ gauge field and the solvable model of Eq. 38 maps to the deconfined phase of the gauge theory (precisely at infinite temperature).

\subsection{A $Q D M$ with an extensive ground state entropy}

So far we have discussed QDM that realize either spontaneous VBC, critical states or RVB liquids. We wish to mention here that these three scenarios may not be the only possible ground states for QDM. In particular, a QDM on the kagome lattice with an extensive ground state entropy was 
recently discussed $\frac{170}{170}$ This model was introduced from the observation that the dimer kinetic energy terms arising from an overlap expansion $(\$ 5.2)$ generally have non trivial signs as soon as resonance loops of different lengths are considered. It was then realized that such signs (which make the QDM no longer appropriate for QMC simulations) can lead to qualitatively new phases, different from VBC or RVB liquids. The Hamiltonian introduced in Ref. 170 is similar to that of Eqs. 3839 except that each resonance loop $\alpha$ is now included with a sign $(-1)^{n_{\alpha}}$ where $n_{\alpha}=3, \cdots, 6$ is the number of dimers involved:

$$
\mathcal{H}=\sum_{h}(-1)^{n_{\alpha}}\left[\left|d_{\alpha}(h)\right\rangle\left\langle\bar{d}_{\alpha}(h)|+| \bar{d}_{\alpha}(h)\right\rangle\left\langle d_{\alpha}(h)\right|\right]
$$

These signs are precisely those appearing in the overlap expansion (at the order of one hexagon) of the Heisenberg model on the kagome lattice. This expansion was carried out by Zeng and Elser ${ }^{171}$ in an insightful paper which laid the basis of the analysis of the kagome antiferromagnet in the first neighbor valence-bonds subspace.

Although not exactly solvable, the Hamiltonian of Eq. 42 was shown to be a dimer liquid (short-range dimer-dimer correlation) and to have a huge ground state degeneracy $\sim 2^{N / 6}=1.122^{N}$ ( $N$ is the number of kagome sites). In addition, several numerical indications pointed to a critical behavior of this system,,$\frac{170}{}$ with a possible algebraic decay of energy-energy correlations. It was argued that the effective QDM describing the singlet dynamics of the spin- $\frac{1}{2}$ Heisenberg antiferromagnet on the kagome lattice could be close (in parameter space) to Eq. 42. If correct, this sheds light on the very large density of singlet states observed at low energy in the numerical spectra of that spin model (see \$7).

\section{Multiple-spin exchange models}

\subsection{Physical realizations of multiple-spin interactions}

\subsubsection{Nuclear magnetism of solid ${ }^{3} \mathrm{He}$}

Solid ${ }^{3} \mathrm{He}$ was the first magnetic system in which the importance of MSE interactions was recognized $172|173| 174$ Due to the large zero-point motion of the atoms about their mean positions, tunneling events during which 2 , 3 or 4 atoms exchange their positions in a cyclic way are frequent. These

${ }^{\mathrm{r}}$ Notice that a one-dimensional analog of Eq. 42 can be defined and exactly maps onto the Ising chain in transverse field at its critical point. 
processes generate an effective interaction between the (nuclear) spins which can be written

$$
\mathcal{H}=\sum_{P}-J_{P}(-1)^{P}\left(P_{\text {spin }}+P_{\text {spin }}^{-1}\right)
$$

where the sum runs over permutations $P, J_{P}>0$ is the exchange frequency of the associated tunneling process (in real space) and $P_{\text {spin }}$ acts on the Hilbert space of spin- $\frac{1}{2}$ located on the site of the crystal. The sign $-(-1)^{P}$ depends of the signature of the permutation $P$ and is a consequence of the Pauli principle. For a cyclic permutation involving $n$ spins this sign is just $(-1)^{n}$ and is responsible for the ferromagnetic character of processes involving an odd number of spins. For spin- $\frac{1}{2}$ particles, two and three-spin exchange terms reduce to the familiar Heisenberg interaction:

$$
\begin{array}{r}
P_{12}=2 \vec{S}_{1} \cdot \vec{S}_{2}+\frac{1}{2} \\
P_{123}+P_{321}=P_{12}+P_{23}+P_{31}-1
\end{array}
$$

but this is no longer true for $n \geq 4$ :

$$
P_{1234}+P_{4321}=P_{12} P_{34}+P_{14} P_{23}-P_{13} P_{24}+P_{13}+P_{24}-1
$$

which can be expressed (thanks to Eq. 44) as a sum of terms with two and four Pauli matrices.

${ }^{3} \mathrm{He}$ can form solid atomic mono-layers with a triangular geometry when adsorbed on a graphite substrate at ultra low temperatures (milli Kelvin range). This $2 \mathrm{D}$ magnet has been studied for a long time (see Refs. 175|176 and references therein) and the importance of MSE interactions involving up to six atoms has now been recognized $177 / 178$ The exchange frequencies of the most important processes have been computed by Path Integral Monte Carlo (PIMC) 179|180|181|182 (analytic WKB calculations have also been carried out $\frac{177 \mid 183}{}$ ) as a function of the density. The proposed MSE Hamiltonian describing the magnetic properties of this $2 \mathrm{D}$ quantum crystal reads

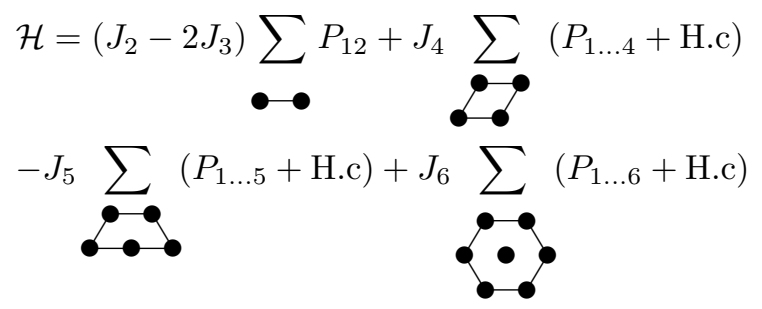


where Eq. 45 was used to absorb the three-spin terms into an effective firstneighbor Heisenberg exchange $J_{2}^{\text {eff }}=J_{2}-2 J_{3}$. At high density the hardcore potential between Helium atoms only leaves three-body exchanges possible $\left(J_{3} \gg J_{n \neq 3}\right)$ and Eq. 48 reduces to a first neighbor Heisenberg ferromagnet, $\frac{184}{18}$ as observed experimentally for the first time by Franco et $a l \cdot \frac{185}{10}$ in high-density layers. On the other hand the second layer solidifies at lower density and higher order exchange terms cannot be ignored PIMC simulations ${ }^{182}$ and high-temperature fits of the experimental data ${ }^{178}$ showed that the relative strength of two- and four-spin terms if roughly $J_{2}^{\text {eff }} / J_{4} \sim-2$ in the low-density second layer solid.

The $J_{2}-J_{4}$ model was studied by exact diagonalizations. It exhibits many distinct phases. There are evidence of a short-range RVB SL phase with no broken symmetry $186|114| 187$ and different gapless exotic phases, with $S U(2)$ symmetry breaking but non on-site magnetization. Exotic order parameters have been evidenced in different part of the phase diagram, as a chiral current ${ }^{397}$ and an octupolar moment 398

The most recent ultra-low temperature measurements of specific heat ${ }^{188}$ and uniform susceptibility ${ }^{189}$ are not incompatible with a gapless phase, but the signature of the octupolar moment remains to be observed ${ }^{399}$

\subsubsection{Wigner crystal}

The Wigner crystal is another fermionic solid with a triangular geometry where MSE interactions can play an important role. At very low density the Coulomb energy dominates, the crystal is almost classical and MSE interactions are very small. Exchange frequencies $J_{P}$ can be computed in this regime by a semi-classical (WKB) approximation $177|190| 191$ and, as for the high density solid of ${ }^{3} \mathrm{He}$, three-body exchanges dominate and give rise to ferromagnetism. However, at higher density and close to melting, PIMC calculations of the exchange frequency ${ }^{\sqrt{192}}$ showed that the magnetism may be described by a MSE model with parameters $\left(J_{2}^{\text {eff }}\right.$ and $\left.J_{4}\right)$ close to those where the triangular MSE model is expected to be a RVB SL. Unlike the ${ }^{3} \mathrm{He}$ case, the particles (electrons) are charged and an external magnetic field has also an orbital effect, it introduces complex phases in the exchange energies: $P+P^{-1} \rightarrow e^{i \alpha} P+e^{-i \alpha} P^{-1}$ where the angle $\alpha=2 \pi \phi / \phi_{0}$ is proportional to the magnetic flux $\phi$ passing through the area enclosed by the exchange trajectory and $\phi_{0}$ is the unit flux quantum. This can give

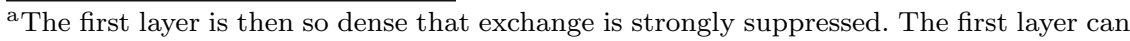
also be replaced by an ${ }^{4} \mathrm{He}$ or HD mono-layer. 
rise to very rich phase diagrams $192 \mid 191$ where complex MSE terms compete with the Zeeman effect (see Ref. 193 for some early experimental attempts to explore this physics).

\subsubsection{Cuprates}

The possibility of significant four-spin exchange around square $\mathrm{Cu}$ plaquettes in copper oxide compounds was first suggested by Roger and Delrieu ${ }^{194}$ They interpreted the anomalously large width of Raman scattering spectra as a signature of four-spin exchange in this copper oxide superconductor. The importance of these MSE interactions in $\mathrm{CuO}_{2}$ planes $\left(J_{4} \sim 0.25 J_{2}\right)$ has then been emphasized by a number of groups and in different materials and by different experimental and theoretical approaches $195|196| 197 \mid 198$ Four-spin plaquette ring exchange also plays a significant role in ladder compounds $199|200| 201 \mid 202$ For instance, exchange parameters with values $J_{\text {rung }}=J_{\text {leg }}=110 \mathrm{meV}$ and $J_{\text {ring }}=16.5 \mathrm{meV}$ were proposed for $\mathrm{La}_{6} \mathrm{Ca}_{8} \mathrm{Cu}_{24} \mathrm{O}_{41}$ based on the dispersion relation of magnetic excitations 199200

\subsection{Two-leg ladders}

Numerous works were devoted to ladder models with four-spin interactions. These include general bi-quadratic interactions as well as models with ringexchange terms. We will only discuss here the simplest of these MSE models:

$$
\begin{aligned}
\mathcal{H} & =J \sum_{n}\left(\vec{S}_{n, 1} \cdot \vec{S}_{n, 2}+\vec{S}_{n, 1} \cdot \vec{S}_{n+1,1}+\vec{S}_{n, 2} \cdot \vec{S}_{n+1,2}\right) \\
& +K \sum_{\square}\left(P_{1234}+H . c\right)
\end{aligned}
$$

Thanks to several studies $200|203| 204|205|$ the phase diagram of this Hamiltonian is now rather well understood and five different phases were identified.

- Ferromagnetic phase. The ground state is fully polarized. This phase includes the $(J=-1, K=0)$ and the $(J=0, K=-1)$ points.

- Rung-singlet phase. This phase includes the ground state of the ladder without MSE term $(J=1, K=0)$. The spectrum is gapped and the ground state is unique. A moderate $K / J \gtrsim 0.23 \pm 0.03$ destroys this phase $200|206| 207 \mid 205]$ in favor of the VBC below.

- Staggered VBC with dimers on the legs. In one of the two degenerate ground states the dimerized bonds are $(2 n, 1)-(2 n+1,1)$ 
and $(2 n+1,2)-(2 n+2,2)$. The VBC disappears for $K / J \gtrsim 0.5 .205$ Such a staggered VBC was first predicted in the framework of a ladder with bi-quadratic interaction by Nersesyan and Tsvelik ${ }^{59}$ Using Matrix-Product Ansatz, Kolezhuk and Mikeska ${ }^{60}$ constructed models which are generalizations of Eq. 48 and which have exact ground state with long-range staggered dimer correlations. In this phase the magnetic excitations are very different from the magnon excitations of the rung-singlet phase above. Here the excitations do not form well-defined quasi-particles but a continuum made of pairs of domain walls connecting two dimerized ground states 5960

- Scalar chirality phase. The order parameter is $\left\langle\vec{S}_{n, 1} \cdot\left(\vec{S}_{n, 2} \times\right.\right.$ $\left.\left.\vec{S}_{n+1,2}\right)\right\rangle$ and it spontaneously breaks the time-reversal symmetry and translation invariance. The ground state is two-fold degenerate up to the next transition at $K / J \simeq 2.8 \pm 0.3{ }^{205}$ There exists a duality transformation ${ }^{204 \mid 208}$ which maps the scalar chirality order parameter onto the dimer order parameter of the VBC above $\mathrm{b}^{\mathrm{b}}$ Applying such a transformation to the exact VBC ground states mentioned above, models with an exactly known ground state and

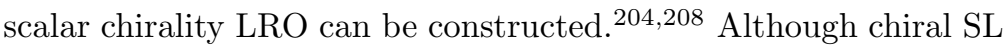
have been much discussed in the literature, this is to our knowledge the first realization of such a phase in a $\mathrm{SU}(2)$ symmetric spin- $\frac{1}{2}$ model.

- Short-range ordered phase with vector-chirality correlations. The strongest correlations are $\left\langle\left(\vec{S}_{n, 1} \times \vec{S}_{n, 2}\right) \cdot\left(\vec{S}_{n^{\prime}, 1} \times \vec{S}_{n^{\prime}, 2}\right)\right\rangle$ but they remain short-range. The spectrum is gapped and the ground state is unique. This phase includes the pure $K=1$ model where $J=0$. This phase is related by the duality transformation discussed above to the rung-singlet phase ${ }^{208}$ This transformation indeed relates the Néel correlations $\left\langle\left(\vec{S}_{n, 1}-\vec{S}_{n, 2}\right) \cdot\left(\vec{S}_{n^{\prime}, 1}-\vec{S}_{n^{\prime}, 2}\right)\right\rangle$ (which are the strongest ones in the rung-singlet phase) to the vector-chirality correlations. Close to the ferromagnetic phase $(J<$ 0 ) one observes a crossover to a region where the strongest correlations are ferromagnetic spin-spin correlations along the legs and antiferromagnetic along the rungs $\frac{205}{205}$

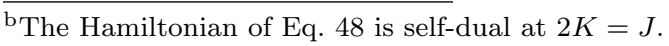




\subsection{MSE model on the square lattice}

The phase diagram of the Hamiltonian 48 on the $2 D$ square-lattice has been recently studied by Läuchli $\frac{209}{}$ by exact diagonalizations. Néel, ferromagnetic, columnar VBC and staggered VBC phases were identified, as in the ladder model above. In addition, a nematic phase characterized by longrange vector chirality correlations (alternating spin currents) was found around the $K=1, J=0$ point. To our knowledge this could be the first microscopic realization of a nematic order in a $2 \mathrm{D}$ spin- $\frac{1}{2}$ model.

\section{4. $R V B$ phase of the triangular $J_{2}-J_{4} M S E$}

Because of its relevance to solid ${ }^{3} \mathrm{He}$ films and Wigner crystals, the MSE model on the triangular lattice has been the subject of many studies $210|211| 212|213| 186|114| 187$ We will discuss here some properties of the simplest MSE model with up to four-spin cyclic exchange interactions $\left(J_{2}-2 J_{3}\right.$ and $J_{4}$ only in Eq. 48). The classical phase diagram (Fig. 9) of this model has been studied by Kubo and collaborators $210 \mid 211$ and the quantum one has been roughly scanned in Ref. $\frac{187}{}$ : we will mainly focus on the short-range RVB spin liquid (see Fig. 99), which might be the first RVB $S L$ encountered in an SU(2)-symmetric spin model.

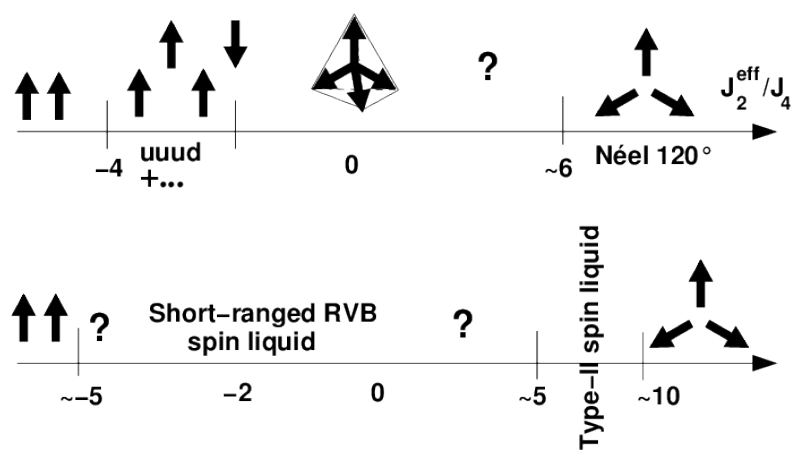

Fig. 9. Classical (top) and quantum (bottom) phases of the $J_{2}-J_{4}$ MSE Hamiltonian. The classical model was studied by Kubo and Momo and is based on a variational approach. The quantum phase diagram is the simplest scenario compatible with the

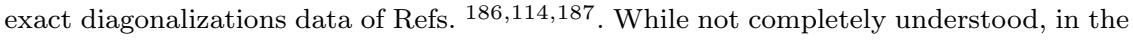
type-II spin-liquid region the spectra are characterized by a very large number of singlet excitations below the first triplet state. This is not the case in the RVB phase. 


\subsubsection{Non-planar classical ground states}

It is well-known that an Heisenberg model (with possible second-neighbors, third-neighbors, ... interactions) on a Bravais lattice always admits a planar helical ground state at the classical level. This is no longer true when MSE are present and finding the classical ground state for arbitrary $J_{2}$ and $J_{4}$ is an unsolved problem. A mean-field phase diagram was obtained for the classical mode $\sqrt{210}$ but very few exact results are known. In the neighborhood of $J_{4}=1 J_{2}=0$ the classical ground state is known to be a four-sublattice configurations with magnetizations pointing toward the vertices of a regular tetrahedron. ${ }^{210}$ This is a quite interesting model where the ground state spontaneously breaks a discrete Ising symmetry associated to the sign of the triple product $\vec{S}_{1} \cdot\left(\vec{S}_{2} \times \vec{S}_{3}\right)$ around a triangle. This broken symmetry gives rise to a finite-temperature phase transition which has been observed in Monte Carlo simulations. ${ }^{211}$ This phenomena is similar to the transition predicted in the $(\pi, 0)$ phase of the $J_{1}-J_{2}$ model on the square lattice. ${ }^{14}$

\subsubsection{Absence of Néel LRO}

The classical ground states at $J_{4}=1, J_{2}=0$ are tetrahedral configurations. Although this phase appears to be stable within the framework of linear spin-wave calculations ${ }^{211}$ or Schwinger-Boson mean-field theory ${ }^{212}$ exact diagonalizations indicate that the magnetic LRO is washed out by quantum fluctuations $211 \mid 114$ The chiral order predicted to survive at long distances and finite temperatures ${ }^{211}$ in the classical system for $J_{2}=0$ is also likely to be washed out by quantum fluctuations. 114

When $J_{2}=1$ a relatively small amount of $J_{4} \sim 0.1$ is sufficient to destroy the three-sublattice Néel LRO realized by the first-neighbor Heisenberg model ${ }^{187}$ The nature of the phase on the other side of this transition is not settled but the finite-size spectra display a large density of singlet excitations at low energy which could be reminiscent of the kagome situation. $\frac{187}{17}$

From exact diagonalizations (up to 36 sites) no sign of Néel LRO could be found at $J_{4}=1, J_{2}=-2 \sqrt{186 / 114}$ In addition, the finite-size analysis showed that the spin-spin correlation length is quite short at $J_{2}=-2$ and $J_{4}=1$ and a spin gap of the order of $\Delta \sim 0.8$ exists at this point. Much of the numerical effort to elucidate the nature of the MSE ground state was concentrated on this point because it is close to the parameters realized in low-density ${ }^{3} \mathrm{He}$ films (when higher order exchanges are neglected). 


\subsubsection{Local singlet-singlet correlations - absence of lattice symmetry breaking}

Having excluded the possibility of a Néel ordered ground state at $J_{4}=$ $1, J_{2}=-2$ it is natural to look for a possible VBC. Because of the complexity of the MSE Hamiltonian it is not clear what kind of spatial order should be favored. From the analysis of dimer-dimer correlations (see Fig. 10 it appears that parallel valence-bonds repel each-other at short distance. This is similar to what is observed in the staggered phase of the $J_{2}-J_{4}$ MSE ladder and square-lattice models. For this reason it appears that a plausible VBC would be the staggered VBC encountered in the triangular QDM for $V>J$ (\$5.5). However this scenario seems difficult to reconcile with the weakness of dimer-dimer correlations. $\frac{114}{14}$ In addition, the low-energy singlet states and their quantum numbers 214 do not reflect the 12-fold quasi-degeneracy that should be present if the system was to spontaneously break some lattice symmetry according to a staggered VBC pattern. Small systems usually favor ordered phases because low-energy and long-wavelength fluctuations that could destabilize an ordered state are reduced compared to larger systems. From the fact that the finite-size spectra do not show the signatures of a staggered VBC symmetry breaking it is unlikely that the MSE model could develop a VBC of this kind in the thermodynamic limit.

\subsubsection{Topological degeneracy and Lieb-Schultz-Mattis Theorem}

Because no VBC phase could be identified in the MSE model at $J_{4}=$ $1, J_{2}=-2$ the numerical data were compared with the predictions of an RVB liquid scenario.

In one dimension a famous theorem due to Lieb, Schultz and Mattis $\$ 215$ (LSM) states that in a one-dimensional spin system with an half-integer spin in the unit cell there is at least on excited state collapsing to the ground state in the thermodynamic limit (periodic boundary conditions). There are in fact several arguments suggesting that this theorem might, at least to some extent, also apply to higher dimensions 216|217|157? ?218 If that is the case a gapped system with an odd integer spin in the unit cell must have a degenerate ground state. The simplest scenario to explain this degeneracy is a translation symmetry breaking. One could think that this would rule out the possibility of any (translation invariant) RVB liquid in such models. This is incorrect because a ground state degeneracy can have a topological origin on a system with periodic boundary conditions, as we discussed in the framework of QDM (\$5). Such a phase is characterized 


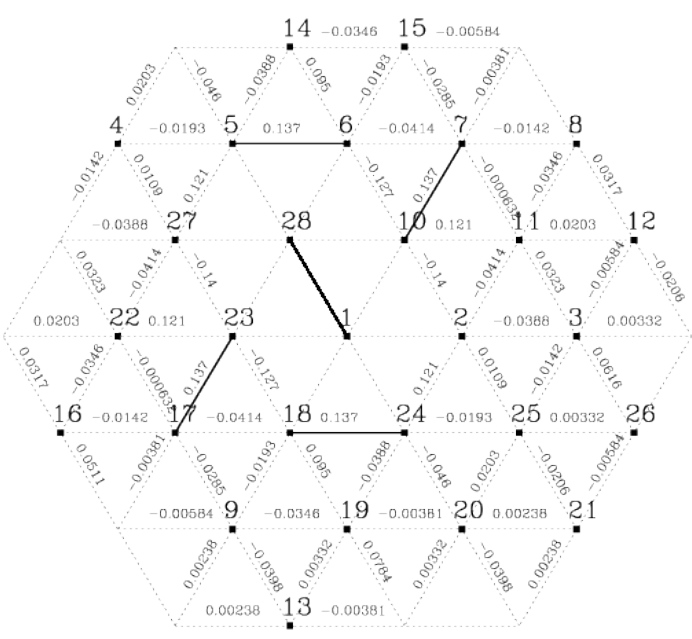

Fig. 10. Dimer-dimer correlations in the ground state of the $J_{2}-J_{4}$ MSE model on the triangular lattice (28 sites) at $J_{2}=-2, J_{4}=1$ (from Ref. 114). Numbers are proportional to $\left\langle\hat{d}_{0} \hat{d}_{x}\right\rangle-\left\langle\hat{d}_{0}\right\rangle\left\langle\hat{d}_{x}\right\rangle$ where the operator $\hat{d}_{x}$ projects onto the singlet space of the bond $x$ and $\hat{d}_{0}$ refers to the reference bond $(1,28)$. These results shows a clear tendency for repulsion between parallel dimers.

by a four-fold topological ground state degeneracy when the system is on a torus. That degeneracy allows the system to fulfill LSM's requirement without any spontaneous translation symmetry breaking. 157

On a finite-size system the topological degeneracy is only approximate but some constraints exist for the quantum numbers (momentum in particular) of the quasi-degenerate multiplet $\frac{157}{}$ A system with periodic boundary conditions with an even number of sites but an odd number of rows is expected to have two ground states with differ by a momentum $\pi$ in the direction parallel to the rows, in close analogy to the LSM theorem in dimension one. The numerical spectra of the MSE model exhibit a set of three singlet energy levels collapsing onto the ground state when the system size is increased ${ }^{114}$ and their quantum number turn out to be consistent with the constraints derived from the general RVB picture. ${ }^{157}$ 


\subsubsection{Deconfined spinons}

The SL phase described above is expected to have deconfined spinons ( $S=\frac{1}{2}$ excitations). These excitations should show up as an incoherent continuum in the spin-spin dynamical structure factor. However such a feature would probably be rather difficult to observe on small 2D lattices, in particular due to the small number of inequivalent $\mathbf{k}$-vectors in the Brillouin zone. On the other hand, the binding energy of two spinons can be evaluated by comparing the ground state energy and the first magnetic excitation energy on even and odd samples. In the case of the MSE model at $J_{4}=1, J_{2}=-2$ the results show the existence of a bound-state (it is more favorable to put two spinons in the same small sample than in separate ones, which is not surprising) but this does not mean that the spinons are confined (contrary to the conclusions of Ref. 114). Interestingly this binding energy seems to go to zero for the largest available sizes (Fig. 11): this might indicate the absence of attraction between spinons for large enough separation and an asymptotic deconfinement.

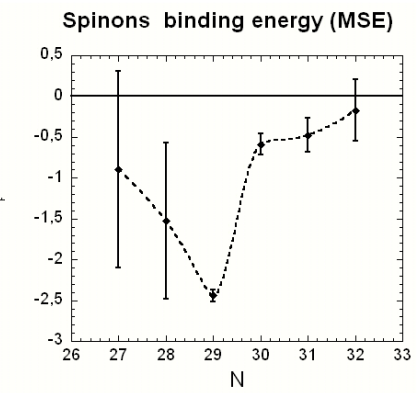

Fig. 11. Spinons binding energy as a function of the system size in the MSE model at $J_{2}=-2$ and $J_{4}=1$. The vertical bars correspond to the range of values found for different sample shapes.

It is important to stress here that the RVB SL phase discussed here (and its QDM counterparts of $\$ 5.5$ and $\$ 5.6$ is not the only way to spinon deconfinement in 2D. There is at least another scenario, inherited from one dimension, which is the sliding Luttinger liquid. Indeed, the Luttinger liquid behavior and the one-dimensional critical behavior of magnetic chains seem to be robust to small (or moderate) frustrating transverse couplings between chains, as observed both in theoretical 219|220|221|222|223|224|225 and numerical approaches $\frac{100}{100}$ This regime between one and two dimensions which may 
have been observed in $\mathrm{Cs}_{2} \mathrm{CuCl}_{4}{ }^{226}$ is the subject of a number of recent studies $222 \mid 223$

\subsection{Other models with MSE interactions}

Multiple-spin interactions are present in a number of models that were found to exhibit fractionalization or an RVB liquid ground state. Well known examples of MSE interactions with an Ising symmetry are $\mathbb{Z}_{2}$ gauge theories, where the gauge invariant plaquette term is a product of Pauli matrices $\prod_{i} \sigma_{i}^{z}$. Such theories have a deconfined phase in $2+1$ dimension ${ }^{169}$ and their relevance to fractionalized phases of $2 \mathrm{D}$ electronic systems has been pointed out by Senthil and Fisher ${ }^{161}$ The connexion between $\mathbb{Z}_{2}$ gauge theories and QDM was mentioned in $\$ 5.6 .7$. Some MSE spin models with an Ising symmetry and a fractionalized ground state were discussed by Kitaev, ${ }^{227}$ Nayak and Shtengel ${ }^{[28}$ In the other limit of a $U(1)$ (or $X Y$ ) symmetry several models have been studied. Recent examples are based upon the spin- $\frac{1}{2}$ four-spin XY ring exchange interaction $229|230| 231 \mid 232$

$$
\mathcal{H}=-K \sum_{\langle i j k l\rangle}\left(S_{i}^{+} S_{j}^{-} S_{k}^{+} S_{l}^{-}+\text {H.c }\right)
$$

which is the $X Y$ analog of the $S U(2)$ MSE interaction $P_{1234}+$ H.c.

\section{Antiferromagnets on the kagome lattice}

The spin- $\frac{1}{2}$ nearest-neighbor Heisenberg model on the kagome lattice has attracted much attention in the long quest of Spin Liquids. The first researches go back to the end of the eighties $244|245| 246|247| 171]$ and more than 300 papers appeared in regular journals since this period. This model possesses the two ingredients that are considered important to obtain a liquid ground state: frustrated geometry (non bipartiteness) and low coordination number which enhance the quantum effects. Moreover the classical model is at a high-degeneracy point in parameter space, where several phases meet. The first breakthrough in the study of this tough quantum problem has been done thanks to large scale exact diagonalisations $\frac{247|171| 248|249| 251}{2}$ These studies could not detect any form of LRO and the model was therefore an interesting candidate for a Spin Liquid. But the subject remained controversial as the sizes of the clusters in exact diagonalisations were very limited (36 spins during nearly two decades, 48 very recently ${ }^{252}$ and a large number of competing hypotheses were considered (\$7.5). A new impetus was recently provided by the discovery of various $\mathrm{Cu}$ compounds exhibiting 
spin liquid behaviors $(\$ 7.6)$. In the following we will first describe the properties of simpler "parent" models (classical model 87.2 Ising model \$7.1. quantum dimer model $\$ 7.3$. We will then give the 2012 results of state of the art DMRG numerical approaches $(87.4$ for the pure nearest-neighbor spin-1/2 Heisenberg model, describe various proposals of nearby phases (in parameter space) and a brief account of the experimental situation.

\subsection{Ising model}

The classical model remains disordered at all temperatures $\lcm{234 \mid 235}$ The system fails to order even at $T=0$ and has a large finite entropy per site: $S_{\mathrm{kag}}^{\mathrm{Ising}}=0.502$, more than half the independent spin value, much larger than the triangular lattice value $S_{\text {tri }}^{\text {Ising }}=0.323$ and of the order of Pauling approximation for independent triangles $S_{\text {Pauling }}=0.501 . \sqrt[233]{ }$ Moessner and Sondhi have studied this Ising model in a transverse magnetic field (the simplest way to include some quantum fluctuations): the model fails to order for any transverse field, at any temperature $145 \mid 236$

\subsection{Classical Heisenberg models on the kagome lattice}

The nearest-neighbor classical $O(3)$ Heisenberg model on the kagome lattice also has a huge ground state degeneracy. This property holds on different lattices with corner sharing units such as the checkerboard lattice or the three dimensional pyrochlore lattice (Moessner and Chalkes 106|107). On all these lattices the nearest-neighbor Heisenberg Hamiltonian can be written as the sum of the square of the total spin $\vec{S}_{\alpha}$ of individual units $\alpha$ (a triangle for the kagome lattice and a tetrahedron in the $2 \mathrm{~d}$ checkerboard or 3 d pyrochlore cases), which share only one vertex. Classical ground states are obtained whenever $\forall \alpha \vec{S}_{\alpha}=\overrightarrow{0}$. This condition fixes the relative positions of the three classical spins of a triangle at 120 degrees from each other in a plane. But it does not fix the relative orientation of the plane of a triad with respect to the planes of the triads on neighboring triangles: the model

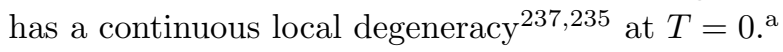

a Counting the planar ground states amounts to determine in how many ways one can associate one of the three letters $A, B$ and $C$ to each site so that each triangle has spins along the three different orientations. This already represents an extensive entropy $\underline{238 \sqrt{235}}$ In such a planar ground state, on may look for a closed loop involving only two spin directions, say $A-B-A-B-\cdots$. By construction, all the spins connected to this loop point in direction $C$. It is then possible to rotate simultaneously all the spins of the 
The classical model has a large density of low-lying excitations at low temperature. ${ }^{243}$ Thermal fluctuations select coplanar configurations because they have the largest phase space for low-energy fluctuations and are therefore entropically favored $237|235| 239 \mid$ The plausibility of long-range order in spin-spin correlations or other observables at very low temperature has also been discussed 235|240|291|292 The recent progresses made in numerical simulations point to a selection of the so-called $\sqrt{3} \times \sqrt{3}$ order at ultra-low temperatures $331 / 375$

The $T=0$ instabilities of the classical model to infinitesimal perturbations has been studied very early ${ }^{281}$ Depending on its sign, a secondneighbor Heisenberg coupling $J_{2}$ leads to two different coplanar phases with unit cells of 3 or 9 spins. They correspond respectively to the solcalled " $q=0$ " and the " $\sqrt{3} \times \sqrt{3}$ " magnetic structures in reference to the order wave vector. The Dzyaloshinsky-Moriya interaction (with DM vector perpendicular to the spin plane) favors the " $q=0$ " phase $241 \mid 242$ It has been recently shown that an infinitesimal antiferromagnetic interaction $J_{3}$ between third neighbors across hexagons favors a non-planar chiral order with a 12-spin unit cell! ${ }^{289}$ Under the action of quantum fluctuations these classical orders can be destabilized to give birth to distinct classes of spin liquids with different fluxes around close contours $286 \mid 376$

A new algebraic method ${ }^{290}$ has been used to enlarge our knowledge of the zero-temperature phase diagram of the classical $J_{1}-J_{2^{-}} J_{3}$ model, as shown in Fig. 12. This method, which is based on symmetry arguments, allows a classification of all the regular classical magnetic orders that can be harboured on a lattice whatever the details of the $S U(2)$ invariant spin Hamiltonian.

\subsection{Nearest-neighbor RVB description of the spin- $\frac{1}{2}$ kagome antiferromagnet}

Assuming that the ground state of the spin- $\frac{1}{2}$ Heisenberg model has a large enough spin-gap, an hypothesis supported by early exact diagonalisations ${ }^{247}$, Zeng and Elser ${ }^{171}$ proposed a variational description of the ground state and low-lying excitations of the kagome antiferromagnet in the basis of nearest neighbor valence bonds. They analyzed in this context the dimer dynamics and showed on a $N=36$ sample that the hexagon VBC -favored by the shortest (three-)dimer moves- melts when

loop by some arbitrary angle about the $C$ axis, without any energy cost. This creates a non-planar ground state. 

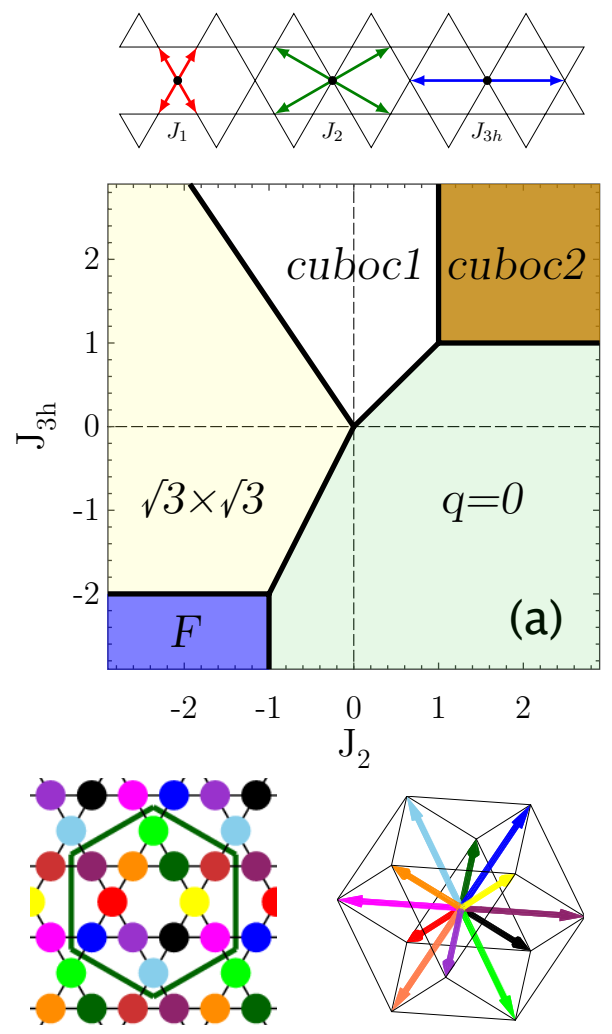

Fig. 12. Phase diagram of the model with up to third neighbors interactions at $T=0$ and $J_{1}=1$. Top: definition of the interactions $J_{1}, J_{2}$ and $J_{3 h}$. Middle: exact classical phase diagram The point $\left(J_{2}, J_{3 h}\right)=(0,0)$ is a tricritical point. Bottom: Description of the cuboc1 order. Left: on the kagome lattice each color corresponds to a different magnetic sublattice. The thick line indicates the 12 site unit-cell. Right: arrows are the spin orientations with the same color coding as in the left figure. The black lines connecting the end of the vectors form a cuboctaedron. On each triangle, the spins are coplanar at 120 degrees; for opposite sites on each hexagon, spins are anti-parallel. The triple products (determinant) of three spins of the hexagons, either first or second neighbors, are non zero and measure the chirality of the phase. They change sign in a mirror symmetry or in a spin flip.

introducing higher order tunneling. Mila and Mambrin $\sqrt{250 \mid 253}$ confirmed that this reduced Hilbert space of next neighbor valence bonds captures some of the perplexing features of this magnet and specifically the absence of (measurable) gap in the singlet sector of small samples. ${ }^{249}$

Some recent numerical results 254 (in the full spin- $\frac{1}{2}$ Hilbert space as 
well as in the RVB subspace) showed that (static) non-magnetic impurities (holes) experience an unexpected repulsion in this system and that no significant magnetic moment is created in the vicinity of the impurities. These non-magnetic impurities provide a valuable probe of spinon confinement in $2 \mathrm{D}$ antiferromagnets 271 and the absence of magnetic moment formation suggest that they are deconfined.

\subsection{Spin- $\frac{1}{2}$ Heisenberg model on the kagome lattice: numerics}

Understanding the nature of the ground state of the nearest neighbor spin$1 / 2$ Heisenberg model on the kagome lattice is clearly a difficult problem: classical degeneracy, numerous competing phases at the quantum level, and sign problem for quantum Monte Carlo methods. The first "unbiased" tool used to approach this problem has been exact diagonalisations. $248|249| 293|338| 294$ This approach has shown the absence of Néel or VBC LRO,$\frac{313}{, 13}$ but the sizes up to $N \leq 36$ were insufficient to give a definite answer on the nature of the spin liquid: a careful analysis has even shown that both a critical gapless spin liquid, as well as a gapped one remained plausible on the basis of the $N=36$ spectra. ${ }^{293}$ In a numerical "tour de force", Läuchli et al. have very recently obtained a part of the low-lying levels of the exact $N=48$ spectrum.252 A refined analysis of these data will probably give some valuable information in a near future.

In the meanwhile a breakthrough in large scale DMRG calculations on very large and long cylinders has brought more insight on the ground state of this puzzling system, as we will explain below. $295|296| 297 \mid 298$

\subsubsection{Ground-state energy per spin}

All these results agree on the extremely low energy per bond of the ground state $\left(\left\langle 2 \vec{S}_{i} \cdot \vec{S}_{j}\right\rangle \simeq-0.438\right) \sim 87 \%$ of the energy per bond in an isolated triangle. On this lattice the energy per bond of the spin- $\frac{1}{2}$ system is much

lower than the classical energy, $\frac{E_{q u}}{E_{c l} .} \sim 1.74$, a ratio much larger than in any other 2D magnet, that can only be compared to the value obtained for the Bethe chain (1.77). The kagome lattice is the 2D lattice which offers the largest stabilization due to quantum fluctuations. 


\subsubsection{Correlations}

Early exact diagonalisations ${ }^{247}$ as well as the latest DMRG simulations 298 found spin-spin and dimer-dimer correlations to be short-ranged, in agreement with the early series expansion. ${ }^{256}$ The scalar chirality correlators $\left\langle\left(\vec{S}_{1} \cdot\left(\vec{S}_{2} \times \vec{S}_{3}\right)\right)\left(\vec{S}_{1^{\prime}} \cdot\left(\vec{S}_{2^{\prime}} \times \vec{S}_{3^{\prime}}\right)\right)\right\rangle$ between elementary triangles have been shown to be short ranged ${ }^{245 / 298}$. Depenbrock et al. added that: "Chiral correlators for other loop types and sizes decay even faster" and will come back to this point later when discussing a putative chiral topological spin Liquid! 302

\subsubsection{Spin gap}

The spin gap of the symmetric $N=36$ toroidal cluster is 0.14 , but the data on such small systems are difficult to extrapolate safely to the thermodynamic limit. The most recent results of DMRG on large and very long cylinders (with diameter up to $\sim 16$ lattice spacings) point to a value of the spin gap $\sim 0 . 1 3 \longdiv { 2 4 9 | 2 9 5 | 2 9 6 | 2 9 7 | 2 9 8 }$ The 2012 belief is thus that nearestneighbor Heisenberg model on the kagome lattice is indeed a gapped spin liquid.

\subsubsection{Singlet gap}

The extension of the Lieb-Shultz Mattis theorem to two dimensions 217/362 guaranties that the spin- $\frac{1}{2}$ kagome antiferromagnet (as any spin model with conserved $S^{z}$, short-range interactions, and a half-odd integer spin per unit cell) cannot simultaneously be gapped and have a unique ground state on a closed surface of genius larger or equal to one. If we admit that spin excitations are indeed gapped, this implies that the spectrum is either gapless in the singlet sector, or the ground state is degenerate in the thermodynamic limit. If the singlet excitations are also gapped, the ground state must be degenerate. If this degeneracy is not the consequence of some conventional lattice symmetry breaking (valence bond crystal for instance), it is said to be a "topological degeneracy" (see 55.5 .2 and $\$ 5.6 .7$ ), and this kind of SL is now called a topological spin liquid.

From the presence of a spin gap and absence of long-range dimer dimer correlations, a topological liquid is thus presently the most natural scenario for the spin- $1 / 2$ Heisenberg problem on the kagome lattice. But the structure of the singlet sector is not completely unveiled at the moment. In a simple $\mathbb{Z}_{2}$ topological spin liquid we expect a four-fold degeneracy on a two 
torus, and a two-fold degeneracy on a cylinder at the thermodynamic limit. In fact, small systems up to 36 sites exhibit a quasi continuum of $S=0$ excitations and absence of a sizeable gap in the singlet sector. $249|293| \mathrm{b}$ There is an interesting evolution of the spectrum between the sizes $N=36$ and $N=48^{252}$ but a coherent picture for the spectrum of low lying excitations obtained in ED remains to be constructed.

On the other hand the first excited singlet state in DMRG is found at a finite energy of about 0.04 or 0.05 above the ground state, 296 which seems contradictory with the Lieb-Shultz-Mattis theorem. There might be different explanations to this: either the splitting induced by the finite cylinder circumference is larger than expected, or the DMRG method is biased toward stabilizing a linear combination of the (quasi-degenerate) ground states with the smallest entanglement entropy. ${ }^{297}$

\subsubsection{Entanglement entropy and signature of a $\mathbb{Z}_{2}$ liquid}

The entanglement entropy (EE) is a powerful tool to detect the presence of "topological order" in states without any conventional local order parameter. In a generic wave function with short-range correlations, the EE $S_{A}$ of a subsystem $A$ has a leading contribution corresponding to the "area law" $S_{A} \simeq a l_{A}^{d-1}$, where $l_{A}$ is the typical linear size of $A$ and $d$ the space dimension. This contribution simply originates from the short-range correlations taking place "across" the boundary of the subsystem $A$. Hamma et al. ${ }^{364}$, Kitaev and Preskil ${ }^{365}$ and Levin and Wen ${ }^{366}$ discovered that, in presence of topological order, the EE may also contain a sub-leading contribution of order $\mathcal{O}\left(l^{0}\right): S_{A} \simeq a l_{A}^{d-1}-\gamma$. This contribution $\gamma$ indicates the presence of some non-local form of quantum entanglement. What makes this sub-leading contribution conceptually interesting and practically very useful is the fact that it is "universal": it depends on the topological phase (through the so-called total quantum dimension), but not on the microscopic or short-distance properties of the wave functions. For a $\mathbb{Z}_{2}$ liquid, the simplest topological phase, we have $\gamma=\ln (2)$.

Extracting $\gamma$ from a given wave function is a non-trivial problem and the original suggestion $\sqrt{365[366}$ was to compute some EE difference between suitably chosen subsystems so that the leading term cancels. This subtraction scheme has been implemented numerically in a few cases $\underline{367|368| 369]}$ It

${ }^{\mathrm{b}}$ This feature, inconsistent with simple pictures of gapped spin liquids, remained puzzling for a long time and may have recently received an explanation. 302 
is however easier and more accurate to obtain $\gamma$ from the EE in a cylinder geometry. There, the translation invariance of the boundary of $A$ allows to extract $\gamma$ simply from a fit to (at least) two system size. The cylinder geometry approach to topological EE has been used successfully in several numerical works, $370|371| 373 \mid 372$ including some on the kagome antiferromagnet with first-neighbor coupling only, ${ }^{298}$ or with additional next-nearest neighbor $J_{2}{ }^{[297}$ For the first-neighbor model, the DMRG results of Ref. 298 gave $\gamma$ consistent with $\ln (2)$ with $\mathrm{a} \simeq 10 \%$ accuracy. With a further neighbor interaction (a point sitting deeper in a possibly distinct liquid phase ${ }^{302}$ ), Ref. ${ }^{297}$ obtained $\gamma \simeq \ln (2)$ with an accuracy of the order of $1 \%$.

\subsubsection{Spin liquids on the kagome lattice and Projective symmetry groups}

X.-G. Wen was the first to develop a constructive approach of fermionic mean-field theories of spin liquids on the square lattice. ${ }^{314}$ The general idea is the following.

In order to be able to describe liquid states with fractionalized excitations (spin-1/2 excitations), it is natural to start with a description in terms of Schwinger bosons or fermions $(44$. Since the microscopic degrees of freedom are instead spin-1 objects, the spinon are inevitably coupled to a gauge field. This gauge field describes the quantum fluctuations of the singlet bonds.

If the effective gauge theory is non-confining the system has spin- $1 / 2$ fractionalized excitations and is a spin liquid. This spin liquid can thus be characterized by the symmetries of the effective theory: space symmetry group (point group and lattice translations), the time reversal symmetry, and the local gauge transformations. Whereas a spin liquid does not break any lattice symmetry, its effective theory in terms of spinons and gauge fields may break the space symmetries insofar as the effect of each space symmetry can be compensated by a gauge transformation, thus achieving an invariant physical spin liquid state. The projective representation of the symmetry group (in short PSG) is defined as the set of all combinations of space and gauge symmetry operations that leave an effective theory (or mean-field Hamiltonian) invariant. The above requirements allow the determination of the distinct mean-field theories describing a fermionic or bosonic spin liquid on a given lattice. This number is finite but may be large (288 fermionic mean-field structures on the square lattice) ${ }^{314}$ This construction does not depend on the details of spin Hamiltonian, except 
for its symmetries. It gives a first general classification of the spin liquids, at least valid in the regime of small gauge fluctuations. It also provides a direct way to determine the low-energy gauge group for a given mean-field state (a particular subset of the PSG, called invariant gauge group (IGG)) ${ }^{\mathrm{C}}$ For a detailed discussion in the context of the kagome lattice, see Ref. 324 .

The PSG approach is a first step toward a classification of spin liquid phases, but many questions remain unanswered ${ }^{d}$ What is the domain of validity of the PSG classification beyond mean field ? How to "measure" the PSG in a spin wave function ? Can two different different PSG correspond to the same phase once fluctuations are included? What is the relation (duality?) between fermionic and bosonic $\mathbb{Z}_{2}$ liquids ?f Is there some spin liquids that cannot be reached by these approaches?

On the kagome lattice Wang et al. studied the PSG of SU(2) invariant hamiltonian in the Schwinger boson representation and found eight different Schwinger boson mean-field Ansaetze of $\mathbb{Z}_{2}$ SLs which preserve all space symmetries (4 of them only have non zero amplitude on nearest neighbor bonds). ${ }^{311} \mathrm{Lu}$ et al ${ }^{312}$ have done the same analysis in the Schwinger fermion representation and found 20 different spin liquids (with only 5 gapped spin liquids with non zero gauge field on $\mathrm{nn}$ bonds). 312 The relation between the $20 \mathbb{Z}_{2}$ SLs in Schwinger fermion representation and the $8 \mathbb{Z}_{2}$ SLs in Schwinger boson representation has not been clarified up to now. The precise gauge structure of the spin liquid exhibited in recent DMRG approaches on the kagome lattice is not known, although two proposals were made in Refs. $\frac{312}{302}$ and

\footnotetext{
c Using the schwinger bosons as a starting point, one generically finds that the low-energy gauge group is $U(1)$ on bipartite lattices, and $\mathbb{Z}_{2}$ on non-bipartite lattices. The former situation leads to confinement (monopole proliferation in the $U(1)$ gauge theory) whereas the situation may or may not - depending if the $\mathbb{Z}_{2}$ gauge theory is in its confined or deconfined phase - correspond to a liquid with deconfined spinons.

d See the oral communications (by M. Hermele, X.-G. Wen, Y. Ran, and others) at the KITP Program: "Frustrated Magnetism and Quantum Spin Liquids: From Theory and Models to Experiments" (Aug 13 - Nov 9, 2012). Available online at: http://online.kitp.ucsb.edu/online/fragnets12

e This question is tackled in Ref. 376, where it is proposed to determine a PSG through the measurement of fluxes which are physical observables, that can be expressed in terms of spin operators.

${ }^{\mathrm{f}}$ This has been anlyzed in details in one case on the honeycomb lattice 318
} 


\subsection{Competing phases}

The classical model is known to be at a high degeneracy point in parameter space [see $\$ 7.2$ and we can expect that perturbations larger than the spin gap and/or larger than the vison gap will destabilize the $\mathbb{Z}_{2}$ Spin liquid in favor of different competing phases. It has been shown for instance that a large enough Dzyaloshinskii-Moriya (DM) coupling (larger than about one tenth the Heisenberg coupling constant) drives the quantum spin liquid towards the $q=0$ semi-classical Néel long-range order $352 \mid 317$ We expect a large enough anti-ferromagnetic second neighbor coupling to have the same effect. Other transitions towards the semi-classical $\sqrt{3} \times \sqrt{3}$ and cuboc1 phases upon increasing ferromagnetic 2nd neighbor or anti-ferromagnetic third neighbor interactions are also expected. But the true nature of these transitions and/or the presence of spin liquid phases in between are today unknown. As explained above the number of possibilities is considerable.

We thus give below a rapid survey of the various states that have been proposed as plausible spin- $1 / 2$ ground states of the Heisenberg problem, as they could be interesting for nearby phases.

\subsubsection{Valence Bond Crystals}

The "simplest" crysta ${ }^{264}$ has a unit cell of 12 spins and is made of resonating stars with 6 dimers. The second VBC, with a unit cell of 36 spins, is made of resonating (trimerized) hexagons. It was discussed as the most reasonable crystal for the pure $J_{1}$ model by Marston and Zeng 147 and Zeng and Elser ${ }^{171}$ and rediscussed more recently $265|266| 267 \mid$ In both scenarios it is the energy gained by local resonances (involving respectively 6 and 3 valence-bonds) which drives the system toward a VBC. From the energy point of view, the star VBC is probably less realistic since it involves a much longer resonance loop. In the pure Heisenberg model the resonance loop involving 6 valence-bonds around a star has a vanishing amplitude at the lowest non-trivial order of the overlap expansion in the RVB subspace, as was shown by Zeng and Elser 171$]$ In the approximation where only the shortest resonance loops are present, the model was indeed found to be in the hexagon VBC phase. A crucial (numerical) result of Zeng and Elser ${ }^{[171}$ is however that at the pure nearest neighbor Heisenberg point this VBC melts when higher order resonances loops are included. Extra couplings would be needed to stabilize this VBC phase. For further reading concerning possible VBC instabilities as well as variationnal approches (tensor networks, RVB subspace, projected fermionic wave functions) see Refs. 303/304/305/306/307. 


\subsection{2. $U(1)$ Dirac Spin Liquid}

Ran et al ${ }^{308}$ have constructed a variational wave function of a gapless $U(1)$ spin liquid. At the mean-field level it corresponds to fermionic spinons with a conical dispersion relation at the Fermi level (Dirac fermions). After Gutzwiller projection this wave function turn out to have a very low energy, even though it has no adjustable parameter. Many correlation functions have an algebraic decay with distance.

The present DMRG results $296 \mid 298$ strongly indicate a spin gap and therefore dismiss this Dirac $U(1)$ scenario but the later may be a good candidate to describe a critical point or even an extended gapless phase nearby. A full study of this interesting phase can be found in Refs. 308|309|310|315|316|325|326

From a completely different starting point (easy plane model), Ryu et $a l\left[{ }^{[327}\right.$ have developed a theory of the singlet sector of a gapless algebraic vortex liquid theory in a XY model on the kagome lattice.

\subsubsection{Spontaneously breaking the time-reversal symmetry, "chiral" spin liquids}

The simplest $\mathbb{Z}_{2}$ topological SL does not break any lattice symmetries nor time reversal. A chiral spin liquid does not break $S U(2)$ symmetry nor translations, but it breaks time reversal symmetry and usually some discrete lattice point-group symmetry. The idea that the kagome lattice can harbour a chiral spin liquid goes back to the end of the eighties 299|288 This idea has revived recently, Wen et al ${ }^{300}$ have studied chiral spin liquids as instability of the Hubbard model and Chua et al ${ }^{301}$ have exhibited an exact gapless spin liquid with stable spin Fermi surface in a Kitaev model on the kagome lattice.

This year Messio et al ${ }^{302}$ have suggested that a chiral $\mathbb{Z}_{2}$ spin liquid could be stabilized in the pure $J_{1}$ Heisenberg model and in the $J_{1}-J_{3}$ model on the kagome lattice. In the classical phase diagram of the $J_{1}-J_{3}$ model an infinitesimal $J_{3}$, 3rd neighbor coupling across the hexagons, lifts the degeneracy of the pure $J_{1}$ model in favor of a twelve sublattice LRO named cuboc1, where the order parameter has the symmetry of a cuboctahedron and the neighboring spins are at $120^{\circ}$. This magnetic structure and its time-reversal counter part (spin inversion) cannot be transformed into each other by a global spin rotation in $S O(3)$. In that sense it is a chiral magnetic order.

Three spins on a nearest neighbor triangle form a plane, but the spins of an hexagon are not planar: the triple product of second neighbor spins 
around an hexagon (scalar chirality) is non zero $\left(\left\langle\vec{S}_{i} \cdot\left(\vec{S}_{j} \times \vec{S}_{k}\right)\right\rangle=0.0148\right)$. Messio's SL state, the spin liquid descendant of this cuboc1 classical structure, is - at the Schwinger boson mean-field level - slightly more stable than the plain $\mathbb{Z}_{2}$ topological SL. It does not break any translation symmetry, but, since its ordered parent is chiral, it breaks time reversal (there is a non trivial flux on the hexagons - 1.e. not 0 nor $\pi$ ) and one reflection symmetry. The above-mentioned chirality has been computed in ED in the ground state of samples with an odd number of spins $(\mathrm{N}=21$ and 27) and found to be sizeable ( 0.817 of the classical value) $\stackrel{249}{\square}$ The 48 -site spectrum obtained by Laüchli et al. ${ }^{252}$ may be compatible with the 8 -fold quasi-degeneracy expected for such a chiral $\mathbb{Z}_{2}$ liquid. DMRG or larger size ED calculations of the associated 6-point correlation functions would be very valuable. It has been conjectured that, due to gauge fluctuations (singlet bond fluctuations), the expectation value of the flux along large loops would obey

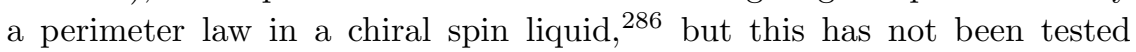
numerically $\mathrm{g}$ Concerning the topological entanglement entropy, the values measured by Jiang et al ${ }^{297}$ and Depenbrock et al ${ }^{298}$ are compatible with a (non-chiral) RVB $\mathbb{Z}_{2}$ SL but the values to be expected in the chiral SL scenario remains to be worked out precisely. If a chiral SL phase does exist at $T=0$, it should undergo a finite temperature a phase transition above which the time-reversal symmetry is restored. The classical problem has been studied, showing a rich interplay of $\mathbb{Z}_{2}$ vortices and chirality domain walls $\longdiv { 3 4 4 3 6 3 }$ but the quantum case has not been studied.

\subsection{Experiments in compounds with kagome-like lattices}

The first experiments on compounds with kagome-like lattices were done on compounds with spin $3 / 2,5 / 2$ or larger. We have no place to recall all these results but it may be remembered that "non-classical" consequences of frustration were very early observed in these compounds. $\mathrm{SrCr}_{9 p} \mathrm{Ga}_{12-9 p} O_{19}$, with the spin- $\frac{3}{2} \mathrm{Cr}$ ions on a kagome lattice (or a bilayer of pyrochlore) has been one of the compounds which have been studied through a large range of techniques. The magnetic excitations of this compound as seen by muons spectroscopy were described as itinerant spins $\frac{1}{2}$ in a "sea of singlets" 272 The non-linear spin susceptibility of $\mathrm{SrCr}_{9} \mathrm{Ga}_{12} \mathrm{O}_{19}$ exhibits a very large increase at about $5 \mathrm{~K}$, reminiscent of spin glasses, but neutrons and muons

g Such an hypothesis on the correlations could perhaps explain the puzzling structure of the exact spectra of small samples as well as the presence of a non zero Chern number for spin- $1 / 2$ excitations. 249 
show that a very significant fraction of the spins are not frozen below this temperature and still exhibit very rapid fluctuations.273 The same phenomena have been observed in two jarosites that are equally good models of kagome antiferromagnets with half-odd-integer spin per unit cell $274 \mid 275$ The low temperature specific heat of these spin systems is unusually large, with a double peak structure. In $\mathrm{SrCr}_{9 p} \mathrm{Ga}_{12-9 p} O_{19}$, it was shown that the low temperature peak was insensitive to large magnetic fields and apparently dominated by singlet states ${ }^{262}$ This might have some relationship with the results of numerical calculations performed on the spin- $\frac{1}{2}$ model 251

The theoretical interest in spin-1/2 compounds on the kagome lattice has lead the experimentalists to a world-wide effort to synthesize new compounds. Volborthite synthesized at the ISSP by Hiroi's group has been the first success. Unhappily, it is not a "perfect" representative of the spatially isotropic Hamiltonian and its modelization is still disputed. This compound has attracted a lot of attention. For a partial bibliography see

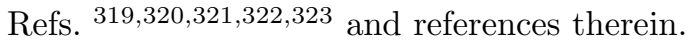

Herbersmithite, with the chemical formula $\mathrm{ZnCu}_{3}(\mathrm{OH})_{6} \mathrm{Cl}_{2}$, a rare mineral identified in 2004 and named after G. F. Herbertsmith (1872-1957) was first synthesized in 2005 in MIT ${ }^{345}$ The spin- $1 / 2 \mathrm{Cu}^{2+}$ ions form $2 \mathrm{D}$ kagome lattices. Inelastic neutron scattering, NMR and $\mu \mathrm{SR}$ consistently show that, in zero or very low magnetic field, Herbersmithite remains fluctuating down to $20 \mathrm{mK} 346|347| 348|349| 354 \mid 342$ This compound is undoubtedly in a SL state but not a perfect realization of the nearest neighbor Heisenberg model as its gap, if it exists, is smaller than $J / 8000$ (where $J$, the nearest neighbor coupling constant, is about 170K). In fact the lack of inversion center on the magnetic bounds allows for Dzyaloshinskii-Moriya interaction of spin orbit origin 334335 These couplings have been estimated of the order of $0 . 0 8 J \longdiv { 3 5 1 / 3 5 5 }$ not far from the theoretically expected quantum critical point ${ }^{[52}$ The role of impurities in such a material has also been theoretically discussed $333[337 / 353$ Single crystals of Herbersmithite are now available and we can expect that future inelastic neutron scattering experiments will uncover new specificities of this spin liquid, and will check more deeply the different theoretical scenarios $308|310| 339$

Many spin-1/2 compounds with the kagome geometry turn out to be

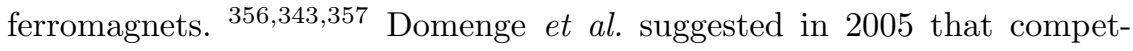
ing interactions (ferromagnetic between nearest neighbors and antiferromagnetic between second neighbors and farther) could lead classically to a non-magnetic phase with a non-planar twelve sublattice magnetic unit cell (called cuboc2) as well as chiral properties ${ }^{\sqrt[358]{3}}$ At $T=0$ this classical phase 
breaks time reversal and spin inversion symmetry and a finite temperature is needed to restore these discrete symmetries. Due to the presence of $\mathbb{Z}_{2}$ vortices, the phase transition is not Ising-like but weakly first order ${ }^{344}$ Messio has recently shown that this phase is the ground state of $J_{1^{-}} J_{2^{-}} J_{3}$ model for a large domain of parameters ${ }^{290}$ The nature of the spin- $1 / 2$ phase of this model is for the moment unknown.

In 2008 a new compound called kapellasite, polymorphous to herbertsmithite, was synthesized in London $\sqrt[359 / 360]{ }$ In this compound the kagome planes are well separated from each other by non magnetic $\mathrm{Zn}^{2+}$ and $\mathrm{Cl}^{-}$ ions. The absence of freezing down to very low temperature as well as NMR and inelastic neutron scattering spectra lead experimentalists to the conclusion that it is a spin liquid $\frac{361}{A b}$ initio calculations suggested that the $J_{1}-J_{2^{-}} J_{3}$ parameters describing this compound were such that in the classical limit the ground state was a chiral and non-planar magnetic structure with the symmetry of a cubocatedron (in this cuboc1 state the spins are at 120 degrees from each other and define a plane on each small triangle, spins around an hexagon are however not coplanar (chirality)) ${ }^{289}$ The inelastic neutron spectra dismissed this hypothesis and suggested that the parent classical phase is instead the cuboc2 phase (this state also has a three-dimensional order parameter with the symmetry of a cubocatedron but neighboring spins are at 60 degrees from each other, there is chirality on the small triangles and the spins around distinct hexagons define distinct planes) ${ }^{\frac{290}{29}}$ A high temperature series analysis of the spin susceptibility and specific heat confirms this hypothesis. $\frac{380}{30}$ The model extracted from these data allows a precise description of the $\mu \mathrm{SR}$ relaxation rate. In spite of a strong disorder there is no signature of "defects". A theoretical explanation for the continuum of excitations seen in this compound is however still lacking.

Two other compounds have been synthesized recently: vesignieite 379 and haydeeite. $\frac{360}{3}$ As herbertsmithite - and in spite of its slight distorsion - vesignieite seems a rather good model of the AF Heisenberg model but it is found to partially order at $T_{c} \sim J / 6 \frac{377 \mid 378}{37}$ This might be mainly due to the presence of DM interactions relatively larger than in herbersmithite. It has been suggested that these two compounds are respectively below (herbersmithite) and above (vesignieite) the quantum critical point separating the spin liquid phase from the $q=0$ Néel ordered phase ${ }^{352}$ Haydeeite, $\alpha-C u_{3} M g(O D)_{6} C l_{2}$, is isostructural to kapellasite $\alpha-C_{3} Z n(O D)_{6} C l_{2}$, but the relative weight of the first neighbor ferromagnetic coupling compared to second and third neighbor antiferromagnetic coupling is apparently 
stronger than in kapellasite and the system is a ferromagnet. 380

\section{Conclusions}


We conclude by summarizing some properties -and related open questions- of the different phases discussed in this review.

The properties of these phases are summarized in Tab. 2. Semi-classical phases with Néel long-range order, magnons as gapless excitations, do exist in spin- $\frac{1}{2} 2 \mathrm{D}$ systems with moderate frustration: the Heisenberg model on the triangular lattice is the most explicit example, with a sublattice magnetization about one half of the classical value. ${ }^{2}$ On a large but finite system the ground state manifold is a tower of states including a number of eigenstates which is a power of the number of sites.

An increased frustration, lower coordination number or smaller spin lead to quantum phases, with a ground state of higher symmetry, no long-range order in spin-spin correlations, a spin gap and the restored $S U(2)$ symmetry. Two main alternatives are then opened: the VBC or VBS phases on one hand, the RVB SL on the other. These states first require the formation of short-range singlets. When a particular local resonance pattern dominates the dynamics of the Hamiltonian the system will try to maximize the number of occurrences of this pattern. This is usually achieved by a regular arrangement, that is a VBC. When no such pattern dominates the system may form a translation invariant RVB SL. In the first case the ground state can be qualitatively described by one ordered configuration of singlets dressed by small fluctuations. In the RVB SL the amplitudes of the wave function are distributed over an exponentially large number of configurations. These ground states lead to very different excitations: $\Delta S=1$ gapped magnons in the first case (and $\Delta S=0$ domain-wall excitations), gapped $\Delta S=0$ visons and gapped $\Delta S=\frac{1}{2}$ unconfined spinons in the second case.

The bosonic large- $N$ results $(4)$ indicate that $\mathrm{VBC}$ are expected in situations where the short range magnetic order is collinear whereas SL appear when these correlation are non-collinear. Although this is verified in many cases (kagome in particular), some exceptions seem to exist also. For instance, the recent DMRG simulations on the $J_{1}-J_{2}$ model on the square lattice ${ }^{382}$ indicate a $\mathbb{Z}_{2}$ SL ground state in the vicinity of $J_{2} / J_{1} \sim 0.5$.

These states obey the 2D extension of LSM theorem $217|362| 388$ if $2 S$ (per unit cell) is odd and if excitations are gapped there must be ground state degeneracy in the thermodynamic limit (with periodic boundary conditions). However the origin of the degeneracy differs in the two types of states. In a VBC the degeneracy is associated to spontaneously broken translation symmetry whereas in the RVB SL the degeneracy has a topo- 
logical origin In the VBS (or explicit VBC) the ground state is unique but $2 S$ is even (in one unit cell). The situation of the hexagonal lattice with respect to this theorem and possible topological degeneracies is interesting: since the lattice has 2 sites per unit cell, the LSM theorem does dot apply. Still, on this lattice, there is no obvious way to construct a gapped and $S U(2)$-symmetric spin- $\frac{1}{2}$ wave function without any conventional nor topological order (see however Ref. ${ }^{389}$ ). Although not covered in this chapter, we mention that some recent quantum Monte Carlo simulations indicating an insulating SL in the (unfrustrated!) Hubbard model at half-filling on the hexagonal lattice ${ }^{390}$ (see also Ref. ${ }^{396}$ ) have triggered a large number of studies concerning frustrated spin models on this lattice $391 / 392$ [393/395

These paradigms are relatively well understood, at least on the qualitative level. They also appear naturally in the broader context of the classification of Mott insulators. ${ }^{[25]}$ However several kinds of $2 \mathrm{D}$ frustrated magnets do not fall in these simple classes and many open questions remain.

This review was restricted to $S U(2)$ invariant Hamiltonians. Whereas the Ising limit has been much studied, the differences between quantum $\mathrm{XY}$ and Heisenberg models have received much less attention.

Chiral SL have not been thoroughly discussed in this review. They are characterized by a broken time-reversal symmetry. This possibility has been studied intensely since the 80 's $286|287| 288 \mid 4$ There might be a revival of this line of research in the future $302 \mid 361$

The issue of quantum phase transitions in frustrated antiferromagnet is also an active topic that is not presented in this review. Many properties of these critical points are still unknown, not to mention the fascinating problems associated with (quenched) disorder.

Limited by place (and competence) we have not discussed in details the works done on spatially anisotropic models. This field which is in between 1D (review by P. Lecheminant in this book) and 2D is extremely flourishing tackled by bosonization and large- $N$ methods.

Ten years ago we wrote in the first edition : "To conclude we would like to emphasize that new analytical and/or numerical methods are highly desirable to proceed in the analysis of the two emblematic problems by which we have opened and closed this review: the $J_{1}-J_{2}$ model on the square lattice and the spin- $\frac{1}{2}$ Heisenberg model on the kagome (and pyrochlore) lattices. In both of these problems a consensus remains to be obtained."

${ }^{a}$ On the kagome lattice (three spins in the unit cell) a precursor of this topological degeneracy might have been seen in the $N=48$-sites spectrum.252 
During the last few years two-dimensional DMRG studies have allowed a breakthrough which has lead to a complete revisiting of these two problems. 296|295|298|297|382] As explained in this rewritten section the full understanding and consensus is not yet reached but a very important step forward has been achieved.

\section{References}

1. E. Manousakis, Rev. Mod. Phys. 63, 1 (1991).

2. B. Bernu, P. Lecheminant, C. Lhuillier and L. Pierre, Phys. Rev. B 50, 10048 (1994).

3. Proceedings of the Highly Frustrated Magnetism 2000 conference, published in J. Can. Phys 79, (2001).

4. E. Fradkin, Field Theories of Condensed Matter Systems, Addison-Wesley (1998).

5. A. Auerbach, Interacting electrons and Quantum Magnetism, SpringerVerlag, Berlin Heidelberg New York, 1994.

6. A. M. Tsvelik, Quantum Field Theory in Condensed Matter Physics Cambridge University Press (1996).

7. S. Sachdev, Quantum Phase Transitions, Cambridge U. Press, New York (1999).

8. J. M. Luttinger and L. Tisza, Phys. Rev. 70, 954 (1946).

9. P. Chandra and B. Douçot, Phys. Rev. B 38, 9335 (1988).

10. J. Villain, R. Bidaux, J. P. Carton and R. Conte, J. Phys. (Paris) 41, 1263 (1980).

11. E. Shender, Sov. Phys. JETP 56, 178 (1982).

12. C. L. Henley, Phys. Rev. Lett. 62, 2056 (1989).

13. A. Moreo, E. Dagotto, T. Jolicœur and J. Riera, Phys. Rev. B 42, 6283 (1990).

14. P. Chandra, P. Coleman, and A. Larkin, Phys. Rev. Lett. 64, 88 (1990).

15. R. R. P. Singh, W. Zheng, J. Oitmaa, O. P. Sushkov, C. J. Hamer, Phys. Rev. Lett. 91, 017201 (2003).

16. G. Misguich, B. Bernu and L. Pierre, Phys. Rev. B 68, 113409 (2003).

17. C. Weber, L. Capriotti, G. Misguich, F. Becca, M. Elhajal and F. Mila, Phys. Rev. Lett. 91, 177202 (2003).

18. T. Jolicœur, E. Dagotto, E. Gagliano and S. Bacci, Phys. Rev. B 42, 4800 (1990).

19. A. Chubukov and T. Jolicœur, Phys. Rev. B 46, 11137 (1992).

20. S. E. Korshunov, Phys. Rev. B 47, 6165 (1993).

21. P. Lecheminant, B. Bernu, C. Lhuillier and L. Pierre, Phys. Rev. B 52, 6647 (1995).

22. R. Melzi et al., Phys. Rev. Lett. 85, 1318 (2000).

23. R. Melzi et al., Phys. Rev. B 64, 024409 (2001).

24. H. Rosner et al., Phys. Rev. Lett. 88, 186405 (2002).

25. H. Rosner et al., Phys. Rev. B 67, 014416 (2003). 
26. D. J. Klein, J. Phys. A: Math. Gen. 15, 661 (1982).

27. C. K. Majumdar and D. K. Ghosh, J. Math. Phys. 10, 1399 (1969).

28. R. R. P. Singh, M. P. Gelfand and D. A. Huse, Phys. Rev. Lett. 61, 2484 (1988).

29. M. P. Gelfand, R. R. P. Singh and D. A. Huse, J. Stat. Phys. 59, 1093 (1990).

30. Zheng Weihong, J. Oitmaa, and C. J. Hamer, Phys. Rev. B 43, 8321 (1991).

31. J. Oitmaa and Zheng Weihong, Phys. Rev. B 54, 3022 (1996).

32. M. P. Gelfand, R. R. P. Singh and D. A. Huse, Phys. Rev. B 40, 10801 (1989).

33. M. P. Gelfand. Phys. Rev. B 42, 8206 (1990).

34. R. R. P. Singh, Zheng Weihong, C. J. Hamer, and J. Oitmaa, Phys. Rev. B 60, 7278 (1999).

35. V. N. Kotov, J. Oitmaa, O. Sushkov and Zheng Weihong, Phil. Mag. B 80, 1483 (2000).

36. M. E. Zhitomirsky and K. Ueda, Phys. Rev. B 54, 9007 (1996).

37. L. Capriotti, F. Becca, A. Parola, and S. Sorella, Phys. Rev. Lett. 87, 097201 (2001).

38. O. P. Sushkov, J. Oitmaa, and Zheng Weihong, Phys. Rev. B 66, 054401 (2002).

39. O. P. Sushkov, J. Oitmaa, and Zheng Weihong, Phys. Rev. B 63, 104420 (2001).

40. M. S. L. du Croo de Jongh, J. M. J. Van Leeuwen, and W. Van Saarloos, Phys. Rev. B 62, 14844 (2000).

41. E. Dagotto and A. Moreo, Phys. Rev. B 39, 4744 (1989), Phys. Rev. Lett. 63, 2148 (1989).

42. F. Figueirido et al., Phys. Rev. B 41, 4619 (1990).

43. D. Poilblanc, E. Gagliano, S. Bacci and E. Dagotto, Phys. Rev. B 43, 10970 (1991).

44. H. J. Schulz, T. A. L. Ziman, Europhys. Lett. 18, 355 (1992). T. Einarsson and H. J. Schulz, Phys. Rev. B 51, 6151 (1995).

H. J. Schulz, T. A. L. Ziman, D. Poilblanc, J. Physique I 6, 675 (1996).

45. S. Chakravarty, B. I. Halperin and D. R. Nelson, Phys. Rev. B 39, 2344 (1989). H. Neuberger and T. Ziman, Phys. Rev. B 39, 2608 (1989). D. Fisher, Phys. Rev. B 39, 11783 (1989). T. Einarsson and H. Johannesson, Phys. Rev. B 43, 5867 (1991). P. Hasenfratz and F. Niedermayer, Z. Phys. B. Condens. Matter 92, 91 (1993).

46. A. W. Sandvik, Phys. Rev. B 56, 11678 (1997).

47. S. Sorella, Phys. Rev. Lett. 80, 4558 (1998).

48. S. Sorella, Phys. Rev. B 64, 024512 (2001).

49. S. White, Phys. Rev. Lett. 69, 2863 (1992).

50. S. White, Phys. Rev. B 48, 10345 (1993).

51. T. Giamarchi and C. Lhuillier, Phys. Rev. B 43, 12943 (1991).

52. B. Shastry and B. Sutherland, Phys. Rev. Lett. 47, 964 (1981).

53. F. Haldane, Phys. Rev. B 25, 4925 (1982).

54. I. Affleck, J. Phys. Cond. Matt. 1, 3047 (1989). 
55. H. Yokoyama and Y. Saiga, J. Phys. Soc. Jpn. 66, 3617 (1997).

56. T. Nakamura and S. Takada, Phys. Rev. B 55, 14413 (1997).

57. D. Augier, E. Sorensen, J. Riera, and D. Poilblanc, Phys. Rev. B 60, 1075 (1999).

58. E. Dagotto and T. M. Rice, Science 271, 618 (1996).

59. A. A. Nersesyan and A. M. Tsvelik, Phys. Rev. Lett. 78, 3939 (1997).

60. A. K. Kolezhuk and H.-J. Mikeska, Int. J. Mod. Phys. B 12, 2325 (1998). Phys. Rev. Lett. 80, 2709 (1998). Phys. Rev. B 56, 11380 (1997).

61. I. Affleck, T. Kennedy, E. Lieb, and H. Tasaki, Phys. Rev. Lett. 59, 799 (1987).

62. I. Affleck, T. Kennedy, E. H. Lieb, and H. Tasaki, Commun. Math. Phys. 115, 477 (1988).

63. M. den Nijs and K. Rommelse, Phys. Rev. B 40, 4709 (1989).

64. T. Kennedy and H. Tasaki, Phys. Rev. B 45, 304 (1992).

65. T. Jolicœur, private communication.

66. K. Hida, J. Phys. Soc. Jpn. 69, 4003 (2000).

67. Y. Yamashita and K. Ueda, Phys. Rev. Lett. 85, 4960 (2000).

68. N. Katoh and M. Imada, J. Phys. Soc. Jpn. 63, 4529 (1994). M. Troyer, H. Tsunetsugu, and D. Wuertz, Phys. Rev. B 50, 13515 (1994). S. Taniguchi et al., J. Phys. Soc. Jpn. 64, 2758 (1995). K. Kodama et al., J. Phys. Soc. Jpn. 65, 1941 (1996). Y. Fukumoto and A. Oguchi, J. Phys. Soc. Jpn. 65, 1440 (1996). K. Kodama et al., J. Phys. Soc. Jpn. 66, 793 (1997). T. Miyasaki and D. Yoshioka, J. Phys. Soc. Jpn. 65, 2370 (1996). T. Ohama, H. Yasuoka, M. Isobe, and Y. Ueda, J. Phys. Soc. Jpn. 66, 23 (1997).

69. K. Ueda, H. Kontani, M. Sigrist, and P. A. Lee, Phys. Rev. Lett. 76, 1932 (1996).

70. M. Albrecht and F. Mila, Phys. Rev. B 53, 2945 (1996).

71. M. Troyer, H. Kontani, and K. Ueda, Phys. Rev. Lett. 76, 3822 (1996).

72. S. Sachdev and N. Read, Phys. Rev. Lett. 77, 4800 (1996).

73. O. A. Starykh et al., Phys. Rev. Lett. 77, 2558 (1996).

74. Zheng Weihong et al., Phys. Rev. B 55, 11377 (1997).

75. H. Kageyama et al., Phys. Rev. Lett. 82, 3168 (1999).

76. H. Nojiri et al., J. Phys. Soc. Jpn. 68, 2906 (1999).

77. S. Miyahara and K. Ueda, Phys. Rev. Lett. 82, 3701 (1999).

78. H. Kageyama et al., Phys. Rev. Lett. 84, 5876 (2000).

79. A. Koga and N. Kawakami, Phys. Rev. Lett. 84, 4461 (2000).

80. E. Müller-Hartmann, R. R. P. Singh, C. Knetter, and G. S. Uhrig, Phys, Rev. Lett. 84, 1808 (2000).

81. K. Totsuka, S. Miyahara, and K. Ueda, Phys. Rev. Lett. 86, 520 (2001).

82. A. Läuchli, S. Wessel, and M. Sigrist, Phys. Rev. B 66, 014401 (2002).

83. K. Kodama et al., Science 298,395 (2002).

84. S. Miyahara and K. Ueda, J. Phys.: Condens. Matter 15, R327-R366 (2003).

85. B. Shastry and B. Sutherland, Physica B (Amsterdam) 108, 1069 (1981).

86. O. Cépas, T. Ziman, Proceedings of the conference in Fukuoka, Nov. 2001. Fukuoka University Press [cond-mat/0207191]. O. Cepas, T. Sakai, T. Ziman, Progr. Theor. Phys. Suppl. 145, 43 (2002). 
87. W. Zheng, J. Oitmaa, and C. J. Hamer Phys. Rev. B 65, 014408 (2002).

88. M. Albrecht and F. Mila, Europhys. Lett. 34, 145 (1996).

89. C. H. Chung, J. B. Marston, and S. Sachdev, Phys. Rev. B 64, 134407 (2001).

90. Y. Fukumoto and A. Oguchi, J. Phys. Soc. Jpn. 68, 3655 (1999).

91. T. Momoi and K. Totsuka, Phys. Rev. B 61, 3231 (2000).

92. T. Momoi and K. Totsuka, Phys. Rev. B 62, 15067 (2000).

93. S. Miyahara and K. Ueda, Phys. Rev. B 61, 3417 (2000).

94. G. Misguich, T. Jolicœur, and S. M. Girvin, Phys. Rev. Lett. 87, 097203 (2001).

95. C. Lhuillier and G. Misguich, in High Magnetic Fields, edited by C. Berthier, L. Levy, and G. Martinez (Springer, Berlin, 2002), pp. 161-190, [condmat/0109146].

96. P. W. Leung and N. Lam, Phys. Rev. B 53, 2213 (1996).

97. J.-B. Fouet, P. Sindzingre, and C. Lhuillier, Eur. Phys. J. B 20, 241 (2001).

98. B. Canals, Phys. Rev. B 65, 184408 (2002).

99. J.-B. Fouet, M. Mambrini, P. Sindzingre, and C. Lhuillier, Phys. Rev. B 67, 054411 (2003).

100. P. Sindzingre, J. B. Fouet, and C. Lhuillier, Phys. Rev. B 66, 174424 (2002).

101. W. Brenig and A. Honecker, Phys. Rev. B 65, 140407R (2002).

102. E. Berg, E. Altman, and A. Auerbach, Phys. Rev. Lett. 90, 147204 (2003).

103. O. Tchernyshyov, O. A. Starykh, R. Moessner and A. G. Abanov, Phys. Rev. B 68, 144422 (2003).

104. R. Moessner, Oleg Tchernyshyov and S. L. Sondhi, cond-mat/0106286.

105. S. Palmer and J. .T. Chalker, Phys. Rev. B 64, 094412 (2002).

106. R. Moessner and J. T. Chalker, Phys. Rev. B 58, 12049 (1998).

107. R. Moessner and J. T. Chalker, Phys. Rev. Lett. 80, 2929 (1998).

108. O. A. Petrenko and D. McK. Paul, Phys. Rev. B 63, 024409 (2001).

109. H. Kawamura and T. Arimori, Phys. Rev. Lett. 88, 077202 (2002)

110. C. L. Henley, Can. J. Phys. (Canada) 79, 1307 (2001). [cond-mat/0009130]

111. J.-B. Fouet, Ph.D. thesis, Université Cergy Pontoise, 2003.

112. M. P. Gelfand et al., Phys. Rev. Lett. 77, 2794 (1996).

113. F. Becca and F. Mila, Phys. Rev. Lett. 89, 037204 (2002).

114. G. Misguich, C. Lhuillier, B. Bernu and C. Waldtmann, Phys. Rev. B 60, 1064 (1999).

115. I. Affleck, Phys. Rev. Lett. 54, 966 (1985).

116. I. Affleck and J. Marston, Phys. Rev. B 37, 3774 (1988).

117. D. Arovas and A. Auerbach, Phys. Rev. B 38, 316 (1988).

118. N. Read and S. Sachdev, Phys. Rev. Lett. 62, 1694 (1989).

119. N. Read and S. Sachdev, Phys. Rev. B 42, 4568 (1990).

120. N. Read and S. Sachdev, Phys. Rev. Lett. 66, 1773 (1991).

121. S. Sachdev and N. Read, Int. J. Mod. Phys. 5, 219 (1991).

122. S. Sachdev in Low Dimensional Quantum Field Theories for Condensed Matter Physicists edited by Y. Lu, S. Lundqvist, and G. Morandi, World Scientific, Singapore, 1995. cond-mat/9303014.

123. N. Read and S. Sachdev, Nucl. Phys. B 316, 609 (1989). 
124. D. Rokhsar, Phys. Rev. B 42, 2526 (1991).

125. K. G. Wilson, Phys. Rev. D 10, 2445 (1974).

126. R. Balian, J. M. Drouffe, and C. Itzykson, Phys. Rev. D 10, 3376 (1974), Phys. Rev. D 11, 2098 (1975), Phys. Rev. D 11, 2098 (1975).

127. D. P. Arovas and A. Auerbach, Phys. Rev. B 38, 316 (1988). A. Auerbach and D. P. Arovas, Phys. Rev. Lett. 61, 617 (1988). J. E. Hirsch and S. Tang, Phys. Rev. B 39, 2850 (1989).

128. H. A. Ceccatto, C. J. Gazza, and A. E. Trumper, Phys. Rev. B 47, 12329 (1993). A. E. Trumper, L. O. Manuel, C. J. Gazza, and H. A. Ceccatto Phys. Rev. Lett. 78, 2216 (1997).

129. We thank O. Tchernyshyov for pointing us this physical interpretation of the $U(1)$ flux.

130. S. Sachdev, Phys. Rev. B 45, 12377 (1992).

131. C. H. Chung and J. B. Marston and Ross H. McKenzie, J. Phys.: Condens. Matter 13, 5159 (2001).

132. F. D. M. Haldane, Phys. Lett. 93A, 464 (1983); Phys. Rev. Lett. 50, 1153 (1983).

133. F. D. M. Haldane, Phys. Rev. Lett. 61, 1029 (1988).

134. X. G. Wen and A. Zee, Phys. Rev. Lett. 61, 1025 (1988). E. Fradkin and M. Stone, Phys. Rev. B 38, 7215 (1988). T. Dombre and N. Read, Phys. Rev. B 38, 7181 (1988).

135. K. Harada, N. Kawashima, and M. Troyer, Phys. Rev. Lett. 90, 117203 (2003).

136. E. Fradkin and S. H. Shenker, Phys. Rev. D 19, 3682 (1979).

137. P. W. Kasteleyn, Physica 27, 1209 (1961).

138. M. E. Fisher, Phys. Rev. 124, 1664 (1961).

139. P. W. Kasteleyn, J. of Math. Phys. 4, 287 (1963).

140. R. Moessner, S. L. Sondhi, E. Fradkin, Phys. Rev. B 65, 024504 (2002).

141. G. Misguich, D. Serban, V. Pasquier, Phys. Rev. Lett. 89, 137202 (2002).

142. R. Moessner, S. L. Sondhi, Phys. Rev. B 68, 054405 (2003).

143. D. S. Rokhsar and S. A. Kivelson, Phys. Rev. Lett. 61, 2376 (1988).

144. R. Moessner and S. L. Sondhi, Phys. Rev. Lett. 86, 1881 (2001).

145. R. Moessner, S. L. Sondhi, and P. Chandra, Phys. Rev. Lett. 84, 4457 (2000).

146. B. Sutherland, Phys. Rev. B 37, 3786 (1988).

147. J. B. Marston, C. Zeng, J. Appl. Phys. 69, 5962 (1991).

148. P. W. Leung, K. C. Chiu and K. J. Runge, Phys. Rev. B 54, 12938 (1996).

149. M. E. Fisher and J. Stephenson Phys. Rev. 132, 1411 (1963).

150. R. Moessner and S. L. Sondhi, Phys. Rev. B 68, 184512 (2003). See Appendix B concerning the square-lattice QDM.

151. R. Moessner, S. L. Sondhi and P. Chandra, Phys. Rev. B 64, 144416 (2001).

152. D. A. Huse, W. Krauth, R. Moessner and S. L. Sondhi, Phys. Rev. Lett. 91, 167004 (2003) and references therein.

153. L. S. Levitov, Phys. Rev. Lett. 64, 92 (1990),

C. L. Henley, J. Stat. Phys. 89, 483 (1997).

154. P. Fendley, R. Moessner and S. L. Sondhi, Phys. Rev. B 66, 214513 (2002). 
155. A. Ioselevich, D. A. Ivanov and M. V. Feigelman, Phys. Rev. B 66, 174405 (2002).

156. L. B. Ioffe, M. V. Feigel'man, A. Ioselevich, D. Ivanov, M. Troyer, G. Blatter, Nature 415, 503 (2002).

157. G. Misguich, C. Lhuillier, M. Mambrini, P. Sindzingre, Eur. Phys. J. B 26, 167 (2002).

158. X. G. Wen, Phys. Rev. B 44, 2664 (1991).

159. S. Kivelson, Phys. Rev. B 39, 259 (1989).

160. N. Read and B. Chakraborty, Phys. Rev. B 40, 7133 (1989).

161. T. Senthil and M. P. A. Fisher, Phys. Rev. B 62, 7850 (2000).

162. T. Senthil and M. P. A. Fisher, Phys. Rev. Lett. 86, 292 (2001); Phys. Rev. B 63, 134521 (2001).

163. R. P. Feynman, Phys. Rev. 90, 1116 (1952), Phys. Rev. 91, 1291 (1953).

164. V. Elser and C. Zeng. Phys. Rev. B 48, 13647 (1993).

165. A. J. Phares and F. J. Wunderlich, Nuovo Cimento B 101, 653 (1988).

166. See $\S$ V.E. 6 of Ref 170

167. This follows from the independence of the arrow variables, see $\S \mathrm{V}$.B of Ref $\frac{170}{170}$

168. A. M. Polyakov, Gauge Fields and Strings, (Harwood Academic, New York, 1987).

169. J. B. Kogut, Rev. Mod. Phys. 51, 659 (1979).

170. G. Misguich, D. Serban and V. Pasquier, Phys. Rev. B 67, 214413 (2003).

171. C. Zeng and V. Elser. Phys. Rev. B 51, 8318 (1995).

172. D. J. Thouless, Proc. Phys. Soc. London 86, 893 (1965).

173. M. Roger, J. H. Hetherington, and J. M. Delrieu, Rev. Mod. Phys. 55, 1 (1983).

174. M. C. Cross and D. S. Fisher, Rev. Mod. Phys. 57, 881 (1985).

175. D. S. Greywall and P. A. Busch, Phys. Rev. Lett. 62, 1868 (1989). D. S. Greywall, Phys. Rev. B 41, 1842 (1990).

176. H. Godfrin and R. E. Rapp, Adv. Phys. 44, 113 (1995).

177. M. Roger, Phys. Rev. B 30, 6432 (1984).

178. M. Roger, C. Bäuerle, Yu. M. Bunkov, A.-S. Chen, and H. Godfrin, Phys. Rev. Lett. 80, 1308 (1998).

179. D. M. Ceperley and G. Jacucci, Phys. Rev. Lett. 58, 1648 (1987).

180. B. Bernu, D. Ceperley, and C. Lhuillier, J. Low Temp. Phys. 89, 589 (1992).

181. D. M. Ceperley, Rev. Mod. Phys. 67, 279 (1995).

182. B. Bernu and D. Ceperley, in Quantum Monte Carlo Methods in Physics and Chemistry, edited by M. P. Nightingale and C. J. Umrigar (Kluwer, Dordrecht, The Netherlands, 1999).

183. H. Ashizawa and D. S. Hirashima, Phys. Rev. B 62, 9413 (2000).

184. J. M. Delrieu, M. Roger, J. H. Hetherington, J. Low Temp. Phys. 40, 71 (1980).

185. H. Franco, R. E. Rapp and H. Godfrin, Phys. Rev. Lett. 57, 1161 (1986).

186. G. Misguich, B. Bernu, C. Lhuillier, and C. Waldtmann, Phys. Rev. Lett. 81, 1098 (1998).

187. W. LiMing, G. Misguich, P. Sindzingre, and C. Lhuillier, Phys. Rev. B 62, 
$6372(2000)$.

188. K. Ishida, M. Morishita, K. Yawata, and H. Fukuyama, Phys. Rev. Lett. 79, 3451 (1997).

189. E. Collin et al., Phys. Rev. Lett. 86, 2447 (2001).

190. M. Katano and D. S. Hirashima, Phys. Rev. B 62, 2573 (2000).

191. D. S. Hirashima and K. Kubo, Phys. Rev. B 63, 125340 (2001).

192. B. Bernu, L. Candido, and D. M. Ceperley Phys. Rev. Lett. 86, 870 (2001).

193. T. Okamoto and S. Kawaji, Phys. Rev. B 57, 9097 (1998).

194. M. Roger and J. M. Delrieu, Phys. Rev. B 39, 2299 (1989).

195. S. Sugai et al., Phys. Rev. B 42, 1045 (1990).

196. R. Coldea et al., Phys. Rev. Lett. 86, 5377 (2001).

197. E. Müller-Hartmann and A. Reischl, Eur. Phys. J. B 28, 173 (2002).

198. A. A. Katanin, and A. P. Kampf, Phys. Rev. B 67, 100404R (2003).

199. M. Matsuda et al., Phys. Rev. B 62, 8903 (2000).

200. S. Brehmer et al., Phys. Rev. B 60, 329 (1999).

201. K. P. Schmidt, C. Knetter and G. S. Uhrig, Europhys. Lett. 56, 877 (2001).

202. A. Gößling et al., Phys. Rev. B 67, 052403 (2003).

203. M. Müller, T. Vekua, and H.-J. Mikeska, Phys. Rev. B 66, 134423 (2002).

204. T. Hikihara, T. Momoi, and X. Hu, Phys. Rev. Lett. 90, 087204 (2003).

205. A. Läuchli, G. Schmid, and M. Troyer, Phys. Rev. B 67, 100409R (2003).

206. Y. Honda and T. Horiguchi, cond-mat/0106426.

207. K. Hijii and K. Nomura Phys. Rev. B 65, 104413 (2002).

208. T. Momoi, T. Hikihara, M. Nakamura, Xiao Hu, Phys. Rev. B 67, 174410 (2003).

209. A. Läuchli, talk given at the Highly Frustrated Magnetism 2003 conference, Grenoble, France (August 2003).

210. K. Kubo and T. Momoi, Z. Phys. B 103, 485 (1997).

211. T. Momoi, K. Kubo, and K. Niki, Phys. Rev. Lett. 79, 2081 (1997).

212. G. Misguich, B. Bernu, and C. Lhuillier, J. Low Temp. Phys. 110, 327 (1998).

213. K. Kubo, H. Sakamoto, T. Momoi, and K. Niki, J. Low Temp. Phys. 111, 583 (1998).

214. A 16- and a 32-site triangular lattices which do not frustrate the staggered VBC were investigated. In both cases some of the irreducible representations of the space group required to break the appropriate lattice symmetries are very high in the spectrum. C. Lhuillier and G. Misguich (unpublished).

215. E. H. Lieb, T. D. Schultz, D. C. Mattis., Ann. Phys. (N.Y) 16, 407 (1961).

216. I. Affleck and E. Lieb, Lett. Math. Phys. 12, 57 (1986).

217. M. Oshikawa, Phys. Rev. Lett. 84, 1535 (2000).

218. M. Oshikawa, Phys. Rev. Lett. 90, 236401 (2003).

219. A. A. Nersesyan, A. O. Gogolin, and F. H. L. Essler, Phys. Rev. Lett. 81, 910 (1998).

220. P. Azaria et al., Phys. Rev. Lett. 81, 1694 (1998).

221. V. J. Emery, E. Fradkin, S. A. Kivelson and T. C. Lubensky, Phys. Rev. Lett. 85, 2160 (2000).

222. M. Bocquet, F. Essler, A. M. Tsvelik and A. O. Gogolin, Phys. Rev. B 
64,094425 (2001), M. Bocquet, Phys. Rev. B 65, 1884415 (2001).

223. A. Vishwanath and D. Carpentier, Phys. Rev. Lett. 86, 676 (2001).

224. S. Sachdev and K. Park, Annals of Physics (N.Y.), 58, 298 (2002).

225. O. A. Starykh and R. R. P. Singh and G. C. Levine, Phys. Rev. Lett. 88, 167203 (2002).

226. R. Coldea, D. A. Tennant, A. M. Tsvelik, and Z. Tylczynski, Phys. Rev. Lett. 86, 1335 (2001).

227. A. Kitaev, Annals Phys. 303, 2 (2003). [quant-ph/9707021]

228. C. Nayak and K. Shtengel, Phys. Rev. B 64, 064422 (2001).

229. L. Balents, M. P. A. Fisher, and S. M. Girvin, Phys. Rev. B 65, 224412 (2002).

230. A. Paramekanti, L. Balents, and M. P. A. Fisher Phys. Rev. B 66, 054526 (2002).

231. A. W. Sandvik, S. Daul, R. R. P. Singh, and D. J. Scalapino, Phys. Rev. Lett. 89, 247201 (2002).

232. T. Senthil and O. Motrunich, Phys. Rev. B 66, 205104 (2002).

233. L. Pauling, in The nature of the chemical bond (Cornell University Press, Ithaca, 1938).

234. K. Kano and S. Naya, Prog. in Theor. Phys. 10, 158 (1953).

235. D. Huse and A. Rutenberg, Phys. Rev. B 45, 7536 (1992).

236. R. Moessner and S. L. Sondhi, Phys. Rev. B 63, 224401 (2001).

237. J. Chalker, P. C. W. Holdsworth, and E. F. Shender, Phys. Rev. Lett. 68, 855 (1992).

238. R. J. Baxter, J. Math. Phys. 11, 784 (1970).

239. I. Richtey, P. Chandra, and P. Coleman, Phys. Rev. B 47, 15342 (1993).

240. J. Reimers and A. Berlinsky, Phys. Rev. B 48, 9539 (1993).

241. M. Elhajal, Ph. D. thesis, Université Joseph Fourier. Grenoble. France, 2002.

242. M. Elhajal, B. Canals, and C. Lacroix, Phys. Rev. B 66, 014422 (2002).

243. A. Keren, Phys. Rev. Lett. 72, 3254 (1994).

244. V. Elser, Phys. Rev. Lett. 62, 2405 (1989).

245. J. Chalker and J. Eastmond, Phys. Rev. B 46, 14201 (1992).

246. S. Sachdev, Phys. Rev. B 45, 12377 (1992).

247. P. Leung and V. Elser, Phys. Rev. B 47, 5459 (1993).

248. P. Lecheminant et al., Phys. Rev. B 56, 2521 (1997).

249. C. Waldtmann et al., Eur. Phys. J. B 2, 501 (1998).

250. F. Mila, Phys. Rev. Lett. 81, 2356 (1998).

251. P. Sindzingre et al., Phys. Rev. Lett. 84, 2953 (2000).

252. A. M. Läuchli, oral communication at the KITP Program: "Frustrated Magnetism and Quantum Spin Liquids: From Theory and Models to Experiments" (Aug 13 - Nov 9, 2012). Available online at: http://online.kitp.ucsb.edu/online/fragnets12/laeuchli Unpublished.

253. M. Mambrini and F. Mila, Eur. Phys. J. B 17, 651 (2001).

254. S. Dommange, M. Mambrini, B. Normand and F. Mila, Phys. Rev. B 68, 224416 (2003)

255. C. Zeng and V. Elser, Phys. Rev. B 42, 8436 (1990). 
256. R. Singh and D. Huse, Phys. Rev. Lett. 68, 1766 (1992).

257. K. Hida, J. Phys. Soc. Jpn. 70, 3673 (2001).

258. D. C. Cabra, M. D. Grynberg, P. C. W. Holdsworth, P. Pujol, Phys. Rev. B 65, 094418 (2002).

259. N. Eltsner and A. P. Young, Phys. Rev. B 50, 6871 (1994).

260. T. Nakamura and S. Miyashita, Phys. Rev. B 52, 9174 (1995).

261. P. Tomczak and J. Richter, Phys. Rev. B 54, 9004 (1996).

262. A. P. Ramirez, B. Hessen, and M. Winkelmann, Phys. Rev. Lett. 84, 2957 (2000).

263. P. Mendels et al., Phys. Rev. Lett. 85, 3496 (2000).

264. A. V. Syromyatnikov and S. V. Maleyev, Phys. Rev. B 66, 132408 (2002).

265. P. Nikolic and T. Senthil, Phys. Rev. B 68, 214415 (2003).

266. R. R. P. Singh and D. A. Huse, Phys. Rev. B 76, 180407(R) (2007).

267. R. R. P. Singh and D. A. Huse, Phys. Rev. B 77, 144415 (2008).

268. C. Mondelli et al., Physica B 284, 1371 (2000).

269. A. Georges, R. Siddhartan and S. Florens, Phys. Rev. Lett. 87, 277203 (2001).

270. C. Lhuillier and P. Sindzingre, in Quantum properties of Low dimensional antiferromagnets, edited by Y. Ajiro and J. P. Boucher (Kyushu University Press, Fukuoka, Japan, 2002), p. 111.

271. S. Sachdev and M. Vojta, Proceedings of the XIII International Congress on Mathematical Physics, July 2000, London. A. Fokas, A. Grigoryan, T. Kibble, and B. Zegarlinski eds, International Press, Boston (2001) [condmat/0009202].

272. Y. Uemura et al., Phys. Rev. Lett. 73, 3306 (1994).

273. S.-H. Lee et al., Europhys. Lett. 35, 127 (1996).

274. A. Keren et al., Phys. Rev. B 53, 6451 (1996).

275. A. S. Wills et al., Europhys. Lett. 42, 325 (1998).

276. K. Awaga et al., Phys. Rev. B 49, 3975 (1994).

277. N. Wada et al., J. Phys. Soc. Jpn. 66, 961 (1997).

278. I. Watanabe et al., Phys. Rev. B 58, 2438 (1998).

279. B. Canals and C. Lacroix, Phys. Rev. Lett. 80, 2933 (1998).

280. A. B. Harris, A. J. Berlinsky and C. Bruder, J. Appl. Phys. 69, 5200 (1991).

281. A.B. Harris, C. Kallin and A.J. Berlinsky, Phys. Rev. B 45, 2899 (1992).

282. H. Tsunetsugu, J. Phys. Soc. Jpn. 70, 640 (2001).

283. H. Tsunetsugu, Phys. Rev. B 65, 024415 (2002).

284. J. V. Delft and C. L. Henley, Phys. Rev. Lett. 69, 3236 (1992), Phys. Rev. B 48, 965 (1993).

285. S. Sachdev, Annals Phys. 303, 226 (2003) [cond-mat/0211027] and Rev. Mod. Phys. 75, 913 (2003).

286. X. Wen, F. Wilczek, and A. Zee, Phys. Rev. B 39, 11413 (1989).

287. V. Kalmeyer and R. Laughlin, Phys. Rev. Lett. 59, 2095 (1987), Phys. Rev. B 39, 11879 (1989).

288. K. Yang, L. Warman, and S. M. Girvin, Phys. Rev. Lett. 70, 2641 (1993).

289. O. Janson, J. Richter, and H. Rosner, Phys. Rev. Lett. 101, 106403 (2008).

290. L. Messio, C. Lhuillier, and G. Misguich, Phys. Rev. B 83, 184401 (2011). 
291. M. E. Zhitomirsky, Phys. Rev. B 78, 094423 (2008).

292. C. L. Henley, Phys. Rev. B 80, 180401 (2009).

293. P. Sindzingre and C. Lhuillier, EPL 8827009 (2009).

294. A. M. Läuchli, J. Sudan, and E. S. Sørensen, Phys. Rev. B 83, 212401 (2011).

295. H. C. Jiang, Z. Y. Weng, and D. N. Sheng, Phys. Rev. Lett. 101, 117203 (2008).

296. S. Yan, D. A. Huse, and S. R. White, Science Magazine 332, 1173 (2011).

297. H.-C. Jiang, Z. Wang, and L. Balents, Nature Physics 8, 902 (2012).

298. S. Depenbrock, I. P. McCulloch, and U. Schollwoeck, Phys. Rev. Lett. 109, 067201 (2012).

299. V. Kalmeyer and R. B. Laughlin, Phys. Rev. B 3911879 (1989).

300. J. Wen, A. Rüegg, C.-C. Wang, G. A Fiete, Phys. Rev. B 82, 075125, (2010).

301. V. Chua, Victor H. Yao and G.A. Fiete, Phys. Rev. B 83, 180412 (2011).

302. L. Messio, B. Bernu, and C. Lhuillier, Phys. Rev. Lett. 108, 207204 (2012).

303. D. Poilblanc, M. Mambrini, and D. Schwandt, Phys. Rev. B 81, 180402 (2010).

304. G. Evenbly and G. Vidal, Phys. Rev. Lett. 104, 187203 (2010).

305. D. Schwandt, M. Mambrini, and D. Poilblanc, Phys. Rev. B 81, 214413 (2010).

306. Y. Iqbal, F. Becca, and D. Poilblanc, Phys. Rev. B 83, 100404 (2011).

307. D. Poilblanc and G. Misguich, Phys. Rev. B 84, 214401 (2011).

308. Y. Ran, M. Hermele, P. A.Lee and X.-G. Wen, Phys. Rev. Lett. 98, 117205 (2007).

309. M. Hermele, T. Senthil and M. P. A. Fisher, Phys. Rev. B 72, 104404 (2005).

310. M. Hermele, Y. Ran, P. A. Lee and X.-G. Wen, Phys. Rev. B 77, 224413 (2008).

311. F. Wang and A. Vishwanath, Phys. Rev. B 74, 174423 (2006).

312. Y.-M. Lu, Y.Ran and P. A.Lee, Phys. Rev. B 83, 224413 (2011).

313. G. Misguich and P. Sindzingre, J. Phys. Cond. Matt., 19, 145202 (2007) and Errata: condmat/0607764v3.

314. X.-G. Wen, Phys. Rev. B 65, 165113 (2002).

315. W.-H. Ko, Z.-X. Liu, T-K Ng and P. A.Lee , Phys. Rev. B 81, 024414 (2010).

316. W.-H. Ko and P. A.Lee, Phys. Rev. B 84, 125102 (2011).

317. L. Messio, O. Cépas and Lhuillier, C., Phys. Rev. B 81, 064428, (2010).

318. Y.-M. Lu and Y. Ran, Phys. Rev. B 84, 024420 (2011).

319. Z. Hiroi, M. Hanawa, N. Kobayashi, M. Nohara, H. Takagi, Y. Kato and M. Takigawa, J. Phys. Soc. Japan, 70, 3377 (2001).

320. Y. Okamoto, M. Tokunaga, H. Yoshida, A. Matsuo, K. Kindo and Z. Hiroi, Phys. Rev. B 83, 180407 (2011).

321. G. J. Nilsen, F. C. Coomer, M. A. de Vries, J. R. Stewart, P. P. Deen, A. Harrison and H. M. Rønnow, Phys. Rev. B 84, 172401, (2011).

322. T. Yavors'kii, W. Apel and H.-U. Everts, Phys. Rev. B 76, 064430 (2007).

323. Fa Wang, A. Vishwanath and Y.B. Kim, Phys. Rev. B 76, 094421 (2007).

324. Y. Huh M. Punk, and S. Sachdev, Phys. Rev. B 84, 094419 (2011). 
325. T. Tay and O.-I. Motrunich,Phys. Rev. B 84, 020404 (2011).

326. Y. Iqbal, F. Becca, D. Poilblanc, Phys. Rev. B 84, 020407 (2011).

327. S. Ryu, O. I. Motrunich, J. Alicea and M. P. A. Fisher, Phys. Rev. B 75, 184406 (2007).

328. T. H. Han, J. S. Helton, S. Chu, A. Prodi, D. K. Singh, C. Mazzoli, P. Müller, D. G. Nocera and Y. S. Lee, Phys. Rev. B 83, 100402 (2011).

329. J.S. Helton, K. Matan, M. P. Shores, E.A. Nytko, B.M. Bartlett,Y. Qiu, D. G. Nocera Y.S. Lee, Phys. Rev. Lett. 104, 147201 (2010).

330. O. Götze, D. J. J. Farnell, R.F. Bishop, P.H. Li and J. Richter, Phys. Rev. B 84, 224428 (2011).

331. O. Cépas and A. Ralko, Phys. Rev. B 84, 020413 (2011).

332. G. Misguich and B. Bernu, Phys. Rev. B 71, 014417 (2005).

333. G. Misguich and P. Sindzingre, Eur. Phys. J. B 59, (2007).

334. M. Rigol and R. R. P. Singh, Phys. Rev. Lett. 98, 207204 (2007).

335. M. Rigol and R. R. P. Singh, Phys. Rev. B 76, 184403 (2007).

336. R. Chitra and M. J. Rozenberg, Phys. Rev.B 77, 052407 (2008).

337. I. Rousochatzakis, S. Manmana, A.M. Läuchli, B. Normand and F. Mila, Phys. Rev. B 79214415 (2009).

338. A. Laeuchli and C. Lhuillier, arXiv:09011065v1.

339. Z. Hao and O. Tchernyshyov, Phys. Rev. B 81, 214445 (2010).

340. F. Bert, S. Nakamae, F. Ladieu,D. L'Hôte, P. Bonville, F. Duc, J.-C. Trombe and P. Mendels, Phys. Rev. B 76, 132411 (2007).

341. E. Kermarrec, P. Mendels, F. Bert R.H. Colman, A.S. Wills, P. Strobel, P. Bonville, A. Hillier and A. Amato, Phys. Rev. B 84, 100401 (2011)

342. M. Jeong, F. Bert, P. Mendels, F. Duc, J. C. Trombe, M. A. de Vries, and A. Harrison, Phys. Rev. Lett. 107, 237201 (2011).

343. Y. Narumi, K. Katsumata,Z. Honda,J.-C. Domenge, P. Sindzingre, C. Lhuillier, Y. Shimaoka, T. C. Kobayashi and K. Kindo, Europhys. Lett. , 65705 (2004).

344. J.-C. Domenge, C. Lhuillier, L. Messio, L. Pierre and P. Viot, Phys. Rev. B 77, 172413 (2008).

345. M. P. Shores, E.A. Nytko, B.M. Bartlett and D. G. Nocera, Journal of the American Chemical Society 127, 13462 (2005).

346. P. Mendels,F. Bert, M. A. de Vries, A. Olariu, A. Harrison, F. Duc, J. C. Trombe, J. S. Lord, A. Amato and C. Baines, Phys. Rev. Lett. 98, 077204 (2007).

347. J. S. Helton, K. Matan, M. P. Shores, E. A. Nytko, B. M. Bartlett, Y. Yoshida, Y. Takano, A. Suslov, Y. Qiu, J.-H. Chung, D. G. Nocera and Y. S. Lee, Phys. Rev. Lett. 98107204 (2007).

348. A. Olariu, P. Mendels, F. Bert, F. Duc,J. C. Trombe, M. A. de Vries and A. Harrison, Phys. Rev. Lett. 100087202 (2008)

349. T. Imai, E. A. Nytko, B. M. Bartlett, M. P. Shores and D. G. Nocera, Phys. Rev. Lett. 100077203 (2008).

350. M. Jeong, F. Bert, P. Mendels, F. Duc, J. C. Trombe, M. A. de Vries and Harrison, A. Phys.Rev. Lett. 107, 237201 (2011).

351. A. Zorko, S. Nellutla, J. van Tol, L.C. Brunel, F. Bert, F. Duc, J. C. Trombe, 
M.A. de Vries, A. Harrison and P. Mendels, Phys. Rev. Lett. 101, 026405 (2008).

352. O. Cépas and C. M. Fong and P. W. Leung and C. Lhuillier, Phys. Rev. B. 78, 140405 (2008).

353. M. A. de Vries, D. Wulferding, P. Lemmens, J.S. Lord, A.Harrison, P. Bonville, F. Bert, and P. Mendels, Phys. Rev. B. 85, 014422 (2012).

354. M.A. de Vries, J. R. Stewart, P. P. Deen, J. O. Piatek, G. J. Nilsen,H. M. Rønnow, A. Harrison, Phys. Rev. Lett. 103, 237201 (2009).

355. S. El Shawish, S. O. Cépas, O. and S. Miyashita, Phys. Rev. B. 81, 224421 (2010).

356. H. Kageyama, T. Nakajima, M. Ichihara, F. Sakai and Y. Ueda, in Spin frustration in two-dimensional compounds edited by Y. Ajiro and J. P. Boucher (Kyushu University Press, Fukuoka, Japan, 2002), p. 135.

357. Y. Karaki, Y. M. Kou, A. Yamaguchi, M. Kubota, H. Ishimoto, Z. Honda, and K. Yamada, J. of Low Temp. Phys. , 158, 653 (2010).

358. J.-C. Domenge, P. Sindzingre, C. Lhuillier and L. Pierre, Phys. Rev. B 72, $024433(2005)$.

359. R. H. Colman, C. Ritter, and A. S. Wills, Chem. Mater. 20, 6897 (2008).

360. R. H. Colman, A. Sinclair, and A. S.Wills, Chem. Mater. 22, 5774 (2010).

361. B. Fak, E. Kermarrec, L. Messio,B. Bernu, B. C. Lhuillier, F. Bert, P. Mendels, B. Koteswararao, F. Bouquet, J. Ollivier, A. D. Hillier, A. Amato, R.H. Colman, A. S. Wills, Phys. Rev. Lett. 109, 037208 (2012).

362. M. B Hastings, Phys. Rev. B 69, 104431 (2004).

363. L. Messio, J.-C. Domenge, C. Lhuillier, L. Pierre, P. Viot and G. Misguich, Phys. Rev. B 78, 054435 (2008).

364. A. Hamma, R. Ionicioiu, and P. Zanardi, Phys. Lett. A 337, 22 (2005).

365. A. Kitaev and J. Preskill, Phys. Rev. Lett. 96, 110404 (2006).

366. M. Levin M and X.-G. Wen, Phys. Rev. Lett. 96, 110405 (2006).

367. S. Furukawa and G. Misguich, Phys. Rev. B 75, 214407 (2007).

368. S. V. Isakov, M. B. Hastings, R. G. Melko, Nature Physics 7, 772 (2011).

369. Yi Zhang, T. Grover, and A. Vishwanath Phys. Rev. B 84, 075128 (2011).

370. A. M. Läuchli, E. J. Bergholtz, M. Haque, New Journal of Physics 12 , 075004 (2010).

371. J.-M. Stéphan, G. Misguich, V. Pasquier, J. Stat. Mech. P02003 (2012).

372. H.C. Jiang, H. Yao, and L. Balents, Phys. Rev. B 86, 024424 (2012).

373. Yi Zhang, T. Grover, A. Turner, M. Oshikawa, A. Vishwanath, Phys. Rev. B 85, 235151 (2012).

374. O. Cépas and A. Ralko, Phys. Rev. B, 84, 020413 (2011).

375. G.-W. Chern and R. Moessner, arXiv:1207.4752.

376. L. Messio, C. Lhuillier and G. Misguich, in preparation.

377. R. H. Colman, F. Bert, D. Boldrin, A. D. Hillier, P. Manuel, P. Mendels and Wills, A. S., Phys. Rev. B 83, 180416 (2011).

378. J. A. Quilliam, F. Bert, F. , R. H. Colman, D. Boldrin, A. S. Wills and P. Mendels, Phys. Rev. B 84, 180401 (2011).

379. Y. Okamoto, H. Yoshida, and Z. Hiroi, J. Phys. Soc. Jpn. 78, 033701 (2009).

380. B. Bernu, E. Kermarrec, L. Messio, C. Lhuillier, F. Bert, and P. Mendels, 
arXiv: 1210.2549 .

381. R. Coldea, D. A. Tennant, A. M. Tsvelik and Z. Tylczynski, Phys. Rev. Lett. 86, 1335 (2001).

382. Hong-Chen Jiang, Hong Yao and Leon Balents, Phys. Rev. B 86, 024424 (2012).

383. Haldane, F. D. M. Phys. Rev. Lett. 61, 2015 (1988).

384. C. L. Kane, C. L. and E.J. Mele, E. J., Phys. Rev. Lett. 95, 146802 (2005).

385. Y. Hatsugai, Phys. Rev. Lett. 71, 3697 (1993).

386. X.-L. Qi, Y.-S. Wu and S.-C. Zhang, Phys. Rev. B 74, 085308 (2006).

387. M. Udagawa and Y. Motome, Phys. Rev. Lett. 104, 106409 (2010).

388. B. Nachtergaele, R. Sims, Commun. Math. Phys. 276, 437 (2007).

389. I. Kimchi, S. A. Parameswaran, A. M. Turner, A. Vishwanath, arXiv:1207.0498.

390. Z. Y. Meng, T. C. Lang, S. Wessel, F. Assaad, A. Muramatsu, Nature 464, 847 (2010).

391. F. Wang, Phys. Rev. B 82, 024419 (2010).

392. A. F. Albuquerque, D. Schwandt, B. Hetényi, S. Capponi, M. Mambrini and A. M. Läuchli, Phys. Rev. B 84024406 (2011).

393. D. C. Cabra, C. A. Lamas, H. D. Rosales, Phys. Rev. B 83094506 (2011).

394. Phys. Rev. Lett. 107, 087204 (2011).

395. H-Y. Yang, A. F. Albuquerque, S. Capponi, A. M. Läuchli, K. P. Schmidt, New J. Phys. 14, 115027 (2012).

396. S. Sorella, Y. Otsuka and S. Yunoki, arXiv:1207.1783.

397. A. Laüchli, J. C. Domenge, C. Lhuillier, P. Sindzingre and M. Troyer, Phys. Rev. Lett. 95, 137206 (2005).

398. T. Momoi, P. Sindzingre and N. Shannon, Phys. Rev. Lett., 97, 257204 (2006)

399. H. Fukuyama, oral communication at the KITP Conference: "Exotic Phases of Frustrated Magnets" (Oct 8-12, 2012). Available online at: http://online.kitp.ucsb.edu/online/fragnets-c12/fukuyama/. Unpublished. 


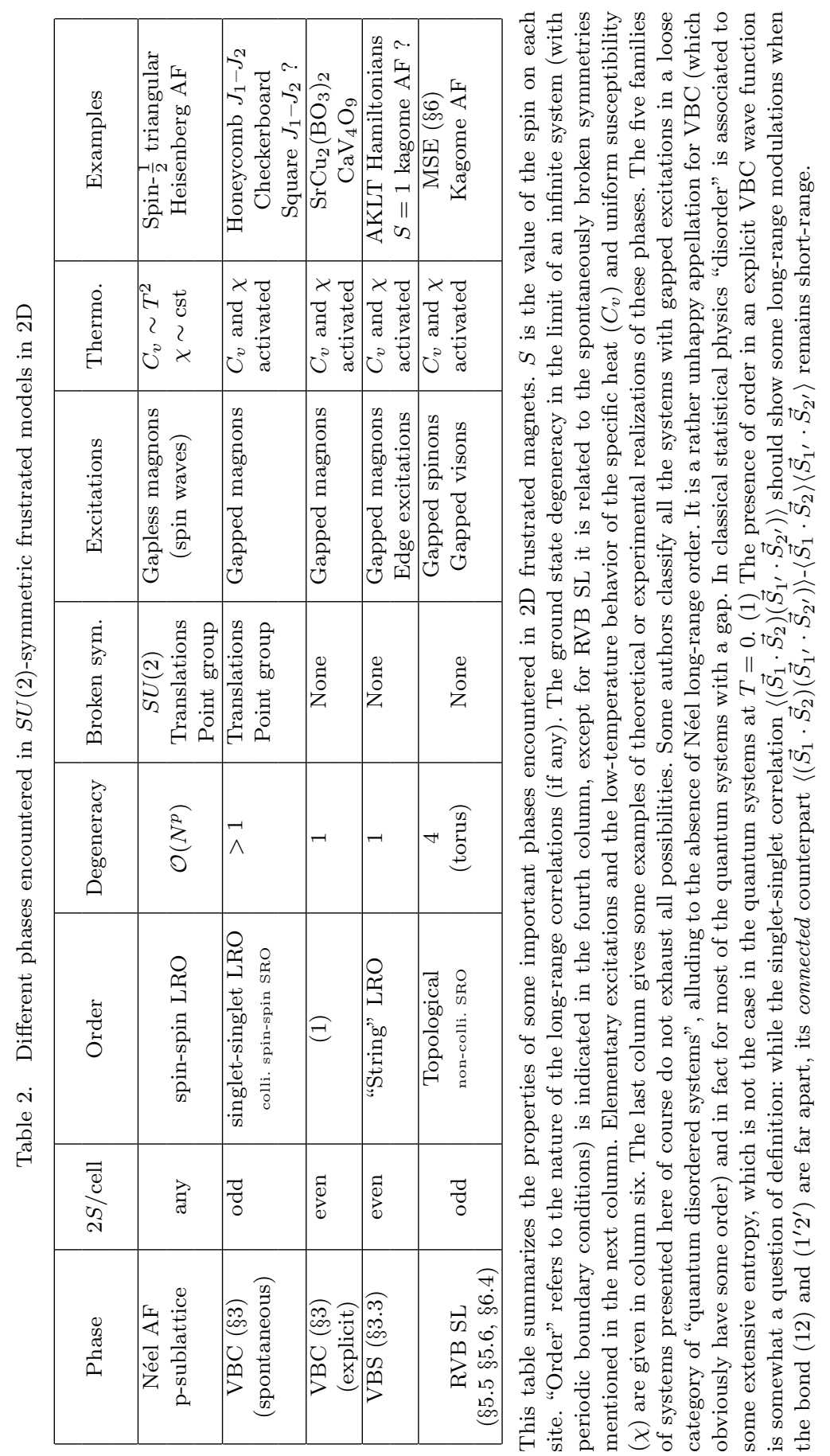

